\title{
Tangent Lie algebra of derived Artin stacks
}

\author{
Benjamin Hennion
}

June 22, 2016

\begin{abstract}
Since the work of Mikhail Kapranov in [Kap], it is known that the shifted tangent complex $\mathbb{T}_{X}[-1]$ of a smooth algebraic variety $X$ is endowed with a weak Lie structure. Moreover any complex of quasi-coherent sheaves on $X$ is endowed with a weak Lie action of this tangent Lie algebra. We will generalize this result to (finite enough) derived Artin stacks, without any smoothness assumption. This in particular applies to singular schemes. This work uses tools of both derived algebraic geometry and $\infty$-category theory.
\end{abstract}

\section{Contents}

$\begin{array}{lr}\text { Introduction } & 1\end{array}$

$1 \quad$ Lie algebras and formal stacks over a cdga $\quad 3$

1.1 Poincaré-Birkhoff-Witt over a cdga in characteristic zero . . . . . . . . . . . . . . . . 3

1.2 Algebraic theory of dg-Lie algebras . . . . . . . . . . . . . . . . . . . . . . . . . . . . . . . . . . . .

1.3 Almost finite cellular objects . . . . . . . . . . . . . . . . . . . . . . . 7

1.4 Homology and cohomology of dg-Lie algebras . . . . . . . . . . . . . . . . . . . . 11

1.5 Formal stack over a dg-algebra . . . . . . . . . . . . . . . . . . . . . . 14

2 Tangent Lie algebra $\quad 17$

2.1 Formal stacks and Lie algebras over a derived Artin stack . . . . . . . . . . . . . . . . 17

2.2 Tangent Lie algebra of a derived Artin stack locally of finite presentation . . . . . . . 19

2.3 Derived categories of formal stacks . . . . . . . . . . . . . . . . . . . . . . . . . . 24

2.4 Atiyah class, modules and tangent maps . . . . . . . . . . . . . . . . . . . . . . . 32

2.5 Adjoint represention . . . . . . . . . . . . . . . . . . . . . 36

$\begin{array}{lr}\text { References } & 40\end{array}$

\section{Introduction}

It is a common knowledge that the shifted tangent complex $\mathbb{T}_{X}[-1]$ of a nice enough geometric stack $X$ in characteristic zero should be endowed with a Lie structure. Moreover any quasi-coherent sheaf on $X$ should admits an action of the Lie algebra $\mathbb{T}_{X}[-1]$ through its Atiyah class. In the article [Kap], Mikhail Kapranov proves the existence of such a structure in (the homotopy category of) the derived category of quasi-coherent sheaves over $X$, when $X$ is a complex manifold. The Lie bracket is there given by the Atiyah class of the tangent complex. The Lie-algebra $\mathbb{T}_{X}[-1]$ should encode the geometric structure of the formal neighbourhood of the diagonal $X \rightarrow X \times X$. Moreover, given a $k$-point of $X$, the pullback of the tangent Lie algebra corresponds to a formal moduli problem as Vladimir Hinich and later Jacob Lurie studied in [Hin] and in [DAG-X]. This formal moduli problem is the formal neighbourhood of the point at hand. Let us also mention the work of Jonathan Pridham in [Pri]. 
In this article we use the tools of derived algebraic geometry (see [TV] for a review) and the machinery of $\infty$-categories (as in [HTT]) to define formal stacks over a derived stack and to study the link with Lie algebras over $X$. This leads to our main theorem.

Theorem 1. Let $X$ be an algebraic derived stack locally of finite presentation over a field $k$ of characteristic zero.

- There is an $\infty$-category $\mathbf{d} \mathbf{S t}_{X}^{\mathrm{f}}$ of formal stacks over $X$ and an adjunction

$$
\mathcal{F}_{X}: \operatorname{dgLie}_{X} \rightleftarrows \operatorname{dSt}_{X}^{\mathrm{f}}: \mathfrak{L}_{X}
$$

- Let $\ell_{X}$ denote the image of the formal completion of the diagonal of $X$ by $\mathfrak{L}_{X}$. The underlying module of $\ell_{X}$ is quasi-isomorphic to the tangent complex of $X$ shifted by -1 (theorem 2.0.1).

- The forgetful functor

$$
\operatorname{dgRep}_{X}\left(\ell_{X}\right) \rightarrow \mathrm{Q} \operatorname{coh}(X)
$$

from representations of $\ell_{X}$ to the derived category of quasi-coherent sheaves over $X$ admits a colimit-preserving section

$$
\operatorname{Rep}_{X}: \operatorname{Qcoh}(X) \rightarrow \operatorname{dgRep}_{X}\left(\ell_{X}\right)
$$

(theorem 2.3.1). Note that $\operatorname{Rep}_{X}\left(\mathbb{T}_{X}[-1]\right)$ is nothing else than the adjoint representation of $\ell_{X}$.

This result specializes to the case of a smooth algebraic variety $X$. It ensures $\mathbb{T}_{X}[-1]$ have a weak Lie structure in the derived category of complexes of quasi-coherent sheaves over $X$. Another consequence is a weak action of $\mathbb{T}_{X}[-1]$ over any complex of quasi-coherent sheaves over $X$, in the sense of [Kap]. Let us also emphasize that the adjunction of the first item is usually not an equivalence. It is when the base $X$ is affine and noetherian.

In the first part of this article, we build an adjunction between formal stacks and dg-Lie algebras when the base is a commutative differential graded algebra $A$ over a field of characteristic zero. In the second part, we define some notion of formal stacks over any derived stack $X$. Gluing the adjunction of part 1, we obtain an adjunction between formal stacks over $X$ and quasi-coherent Lie algebras over $X$. We then prove the existence of the Lie structure $\ell_{X}$ on $\mathbb{T}_{X}[-1]$. The last pages deal with the action of $\ell_{X}$ on any quasi-coherent sheaf over $X$, and compare this action with the Atiyah class.

The author is grateful to Bertrand Toën, Damien Calaque and to Marco Robalo for the many interesting discussions we had about this article. He would also like to thank Mathieu Anel and Dimitri Ara.

A bit of conventions. Throughout this article $k$ will be a field of characteristic zero. Let us also fix two universes $\mathbb{U} \in \mathbb{V}$. Every dg-module, algebra or so will be assumed $\mathbb{U}$-small. We will use the toolbox about $\infty$-categories from [HTT]. We will borrow a bunch of notations from loc. cit. : let $\mathbf{C a t}_{\infty}^{\mathbb{U}}$ denote the $(\infty, 1)$-category of $\mathbb{U}$-small $(\infty, 1)$-categories ; let $\mathbf{P r}_{\infty}^{\mathrm{L}, \mathbb{U}}$ denote the $(\infty, 1)$-category of $\mathbb{U}$-presentable (hence $\mathbb{V}$-small) $(\infty, 1)$-categories with left adjoint functors as morphisms. Every time a category is presentable, it will implicitly mean $\mathbb{U}$-presentable. Given $A \in \mathbf{c d g a}_{k}^{\leqslant 0}$, we will use the following notations

- The $(\infty, 1)$-category $\operatorname{dgMod}_{A}$ of (unbounded) dg-modules over $A$;

- The $(\infty, 1)$-category $\mathbf{c d g a}_{A}$ of (unbounded) commutative dg-algebras over $A$;

- The $(\infty, 1)$-category $\mathbf{c d g a}_{A}^{\leqslant 0}$ of commutative dg-algebras over $A$ cohomologically concentrated in non positive degree ;

- The $(\infty, 1)$-category $\mathbf{d g} \mathbf{A} \lg _{A}$ of (neither bounded nor commutative) dg-algebras over $A$;

- The $(\infty, 1)$-category $\mathbf{d g L i e}$ of (unbounded) dg-Lie algebras over $A$. 
Each one of those $(\infty, 1)$-categories appears as the underlying $(\infty, 1)$-category of a model category. We will denote by $\operatorname{dgMod}_{A}, \operatorname{cdga}_{A}, \operatorname{cdga}_{A}^{\leqslant 0}, \operatorname{dgAlg}_{A}$ and $\operatorname{dgLie}_{A}$ the model categories. To any $(\infty, 1)-$ category $\mathcal{C}$ with finite coproducts, we will associate $\mathcal{P}_{\Sigma}(\mathcal{C})$, the $(\infty, 1)$-category of free sifted colimits in $\mathcal{C}$ - see [HTT, 5.5.8.8]. Recall that it is the category of functors $\mathcal{C}^{\mathrm{op}} \rightarrow$ sSets which preserve finite products.

We will also make use of the theory of symmetric monoidal $(\infty, 1)$-categories as developed in [HAlg]. Let us give a (very) quick review of those objects.

Definition 0.0.1. Let Fin* denote the category of pointed finite sets. For any $n \in \mathbb{N}$, we will denote by $\langle n\rangle$ the set $\{*, 1, \ldots, n\}$ pointed at $*$. For any $n$ and $i \leqslant n$, the Kronecker map $\delta^{i}:\langle n\rangle \rightarrow\langle 1\rangle$ is defined by $\delta^{i}(j)=1$ if $j=i$ and $\delta^{i}(j)=*$ otherwise.

Definition 0.0.2. (see [HAlg, 2.0.0.7]) Let $\mathcal{C}$ be an $(\infty, 1)$-category. A symmetric monoidal structure on $\mathcal{C}$ is the datum of a coCartesian fibration $p: \mathcal{C}^{\otimes} \rightarrow$ Fin* such that

- The fibre category $\mathcal{C}_{\langle 1\rangle}^{\otimes}$ is equivalent to $\mathcal{C}$ and

- For any $n$, the Kronecker maps induce an equivalence $\mathcal{C}_{\langle n\rangle}^{\otimes} \rightarrow\left(\mathcal{C}_{\langle 1\rangle}^{\otimes}\right)^{n} \simeq \mathcal{C}^{n}$.

where $\mathcal{C}_{\langle n\rangle}^{\otimes}$ denote the fibre of $p$ at $\langle n\rangle$. We will denote by $\mathbf{C a t}_{\infty}^{\otimes, \mathbb{V}}$ the $(\infty, 1)$-category of $\mathbb{V}$-small symmetric monoidal $(\infty, 1)$-categories - see [HAlg, 2.1.4.13].

Such a coCartesian fibration is classified by a functor $\phi:$ Fin $^{*} \rightarrow$ Cat $_{\infty}^{\mathbb{V}}-$ see [HTT, 3.3.2.2] - such that $\phi(\langle n\rangle) \simeq \mathcal{C}^{n}$. The tensor product on $\mathcal{C}$ is induced by the map of pointed finite sets $\mu:\langle 2\rangle \rightarrow\langle 1\rangle$ mapping both 1 and 2 to 1

$$
\otimes=\phi(\mu): \mathcal{C}^{2} \rightarrow \mathcal{C}
$$

\section{Lie algebras and formal stacks over a cdga}

In this part we will mimic a construction found in Lurie's [DAG-X]

Theorem 1.0.1 (Lurie). Let $k$ be a field of characteristic zero. There is an adjunction of $(\infty, 1)$ categories:

$$
\mathrm{C}_{k}: \operatorname{dgLie}_{k} \rightleftarrows\left(\operatorname{cdga}_{k} / k\right)^{\mathrm{op}}: \mathrm{D}_{k}
$$

Whenever $L$ is a dg-Lie algebra:

(i) If $L$ is freely generated by a dg-module $V$ then the algebra $\mathrm{C}_{k}(L)$ is equivalent to the trivial square zero extension $k \oplus V^{\vee}[-1]-$ see $[D A G-X$, 2.2.7].

(ii) If $L$ is concentrated in positive degree and every vector space $L^{n}$ is finite dimensional, then the adjunction morphism $L \rightarrow \mathrm{D}_{k} \mathrm{C}_{k} L$ is an equivalence - see [DAG-X, 2.3.5].

The goal is to extend this result to more general basis, namely a commutative dg-algebra over $k$ concentrated in non positive degree. The existence of the adjunction and the point (i) will be proved over any basis, the analog of point (ii) will need the base dg-algebra to be noetherian.

Throughout this section, $A$ will be a commutative dg-algebra concentrated in non-positive degree over the base field $k$ (still of characteristic zero).

\subsection{Poincaré-Birkhoff-Witt over a cdga in characteristic zero}

In this first part, we prove the PBW-theorem over a cdga of characteristic 0 . The proof is a simple generalisation of that of Paul M. Cohn over a algebra in characteristic 0 - see [Coh, theorem 2]. 
Theorem 1.1.1. Let $A$ be a commutative dg-algebra over a field $k$ of characteristic zero. For any $d g$-Lie algebra $L$ over $A$, there is a natural isomorphism of $A$-dg-modules

$$
\operatorname{Sym}_{A} L \rightarrow \mathcal{U}_{A} L
$$

Proof. Recall that $\mathcal{U}_{A} L$ can be endowed with a bialgebra structure such that an element of $L$ is primitive in $\mathcal{U}_{A} L$. The morphism $L \rightarrow \mathcal{U}_{A} L$ therefore induces a morphism of dg-bialgebras $\mathrm{T}_{A} L \rightarrow$ $\mathcal{U}_{A} L$ which can be composed with the symmetrization map $\operatorname{Sym}_{A} L \rightarrow \mathrm{T}_{A} L$ given by

$$
x_{1} \otimes \ldots \otimes x_{n} \mapsto \frac{1}{n !} \sum_{\sigma} \varepsilon(\sigma, \bar{x}) x_{\sigma(1)} \otimes \ldots \otimes x_{\sigma(n)}
$$

where $\sigma$ varies in the permutation group $\mathfrak{S}_{n}$ and where $\varepsilon(-, \bar{x})$ is a group morphism $\mathfrak{S}_{n} \rightarrow\{-1,+1\}$ determined by the value on the permutations $(i j)$

$$
\varepsilon((i j), \bar{x})=(-1)^{\left|x_{i}\right|\left|x_{j}\right|}
$$

We finally get a morphism of $A$-dg-coalgebras $\phi: \operatorname{Sym}_{A} L \rightarrow \mathcal{U}_{A} L$. Let us take $n \geqslant 1$ and let us assume that the image of $\phi$ contains $\mathcal{U}_{A}^{\leqslant n-1} L$. The image of a symmetric tensor

$$
x_{1} \stackrel{s}{\otimes} \ldots \stackrel{s}{\otimes} x_{n}
$$

by $\phi$ is the class

$$
\left[\frac{1}{n !} \sum_{\sigma} \varepsilon(\sigma, \bar{x}) x_{\sigma(1)} \otimes \ldots \otimes x_{\sigma(n)}\right]
$$

which can be rewritten

$$
\left[x_{1} \otimes \ldots \otimes x_{n}+\sum_{\alpha} \pm \frac{1}{n !} y_{1}^{\alpha} \otimes \ldots \otimes y_{n-1}^{\alpha}\right]
$$

where $y_{i}^{\alpha}$ is either some of the $x_{j}$ 's or some bracket $\left[x_{j}, x_{k}\right]$. This implies that $\mathcal{U}_{A}^{\leqslant n} L$ is in the image of $\phi$ and we therefore show recursively that $\phi$ is surjective (the filtration of $\mathcal{U}_{A} L$ is exhaustive).

There is moreover a section

$$
\mathcal{U}_{A} L \rightarrow \operatorname{Sym}_{A} L
$$

for which a formula is given in [Coh] and which concludes the proof.

\subsection{Algebraic theory of dg-Lie algebras}

Let us consider the adjunction Free $_{A}: \mathbf{d g L i e}_{A} \rightleftarrows \operatorname{dgMod}_{A}:$ Forget $_{A}$ of $(\infty, 1)$-categories.

Definition 1.2.1. Let $\operatorname{dgMod}_{A}^{\mathrm{f}, \mathrm{ft}, \geqslant 1}$ denote the full sub-category of $\mathbf{d g M o d} \operatorname{spanned~}_{A}$ by the free dg-modules of finite type whose generators are in positive degree. An object of $\operatorname{dgMod}_{A}^{\mathrm{f}, \mathrm{ft}, \geqslant 1}$ is thus (equivalent to) the dg-module

$$
\bigoplus_{i=1}^{n} A^{p_{i}}[-i]
$$

for some $n \geqslant 1$ and some family $\left(p_{1}, \ldots, p_{n}\right)$ of non negative integers.

Let $\operatorname{dgLie}_{A}^{\mathrm{f}, \mathrm{ft}, \geqslant 1}$ denote the essential image of $\operatorname{dgMod}_{A}^{\mathrm{f}, \mathrm{ft}, \geqslant 1}$ in $\mathbf{d g L i e}_{A}$ by the functor Free.

Let us recall that $\mathcal{P}_{\Sigma}(\mathcal{C})$ stands for the sifted completion of a category $\mathcal{C}$ with finite coproducts. It is equivalent to the category of functors $\mathcal{C}^{\text {op }} \rightarrow$ sSets mapping finite coproducts to products. If $\mathcal{C}$ is pointed, we will denote by $\mathcal{P}_{\Sigma}^{\text {st }}(\mathcal{C})$ the full subcategory of $\mathcal{P}_{\Sigma}(\mathcal{C})$ spanned by those functors $f$ which also map suspensions which exist in $\mathcal{C}$ to loop spaces:

$$
f(0 \underset{c}{\amalg} 0) \simeq * \underset{f(c)}{\times} *
$$


Proposition 1.2.2. The Yoneda functors

$$
\begin{aligned}
& \operatorname{dgMod}_{A} \rightarrow \mathcal{P}_{\Sigma}^{\mathrm{st}}\left(\operatorname{dgMod}_{A}^{\mathrm{f}, \mathrm{ft}, \geqslant 1}\right) \\
& \operatorname{dgLie}_{A} \rightarrow \mathcal{P}_{\Sigma}^{\mathrm{st}}\left(\operatorname{dgLie}_{A}^{\mathrm{f}, \mathrm{ft}, \geqslant 1}\right)
\end{aligned}
$$

are equivalences.

Remark 1.2.3. The above proposition implies that every dg-Lie algebra is colimit of a sifted diagram of objects in $\operatorname{dgLie}_{A}^{\mathrm{f}, \mathrm{ft}, \geqslant 1}$.

Proof. Let us denote by $\mathcal{C}$ the category $\mathbf{d g} \operatorname{Mod}{ }_{A}$ and $\mathcal{C}_{0}=\mathbf{d g M o d}_{A}^{\mathrm{f}, \mathrm{ft}, \geqslant 1}$. We consider the set $S$ of the canonical maps colim $(\operatorname{Nerve}(0 \rightarrow E)) \rightarrow E$ in $\mathcal{P}_{\Sigma}\left(\mathcal{C}_{0}\right)$, where $E$ varies in $\mathcal{C}_{0}$. Let us define $\mathcal{D}$ as the full subcategory of $\mathcal{P}_{\Sigma}\left(\mathcal{C}_{0}\right)$ spanned by $S$-local objects. By construction, the category $\mathcal{D}$ is exactly $\mathcal{P}_{\Sigma}^{\text {st }}\left(\mathcal{C}_{0}\right)$.

It then follows from [HTT, 5.5.7.3] that $\mathcal{D}$ is compactly generated and that any compact object $x$ is obtained as a sifted colimit of objects in

$$
\mathcal{C}_{0}^{\leqslant n}=\left\{A^{p}[-i], p \in \mathbb{N}, 1 \leqslant i \leqslant n\right\} \subset \mathcal{C}_{0}
$$

for some $n$ (depending on $x$ ).

We now consider the natural adjunction $g: \mathcal{D} \rightleftarrows \mathcal{C}: f$ where $f$ is the Yoneda embedding followed by the restriction to $\mathcal{C}_{0}$. The functor $f$ is obviously conservative (as we can functorially retrieve the cohomology groups of a dg-module $M$ out of $f(M)$ ). Let us hence prove that $g$ is fully faithful. To do so, we fix an object $F \in \mathcal{D}$ and study the adjunction map $F \rightarrow f g F$. Since both $f$ and $g$ preserve filtered colimits and since $\mathcal{D}$ is compactly generated, we may assume that $F$ is compact. In particular, the functor $F$ is determined by its restriction to $\mathcal{C}_{0}^{\leqslant n}$ for some $n$. Moreover the image $f g F$ is also determined by its restriction to $\mathcal{C}_{0}^{\leqslant n}$ (this follows from the fact that $g F$ is bounded above) for some $n$. We can hence test for any $E \in \mathcal{C}_{0}^{\leqslant n}$ :

$$
F(E)=\operatorname{colim}_{N \rightarrow F} \operatorname{Map}_{\mathcal{C}}(E, N) \simeq \operatorname{Map}_{\mathcal{C}}(E, \operatorname{colim} N)=\operatorname{Map}_{\mathcal{C}}(E, g F)=f g F(E)
$$

where $N \in \mathcal{C}_{0}^{\leqslant n}$. This concludes the case of $\operatorname{dgMod} \operatorname{Mod}_{A}$.

The forgetful functor Forget ${ }_{A}: \mathbf{d g L i e}_{A} \rightarrow \mathbf{d g M o d}$ is by definition conservative. Moreover, the Poincaré-Birkhoff-Witt theorem 1.1.1 implies that Forget $_{A}$ is a retract of the composite functor

$$
\operatorname{dgLie}_{A} \stackrel{\mathcal{U}_{A}}{\longrightarrow} \operatorname{dg} \operatorname{Alg}_{A} \longrightarrow \operatorname{dgMod} \operatorname{dod}_{A}
$$

Since the latter preserves sifted colimits (the functor $\mathcal{U}_{A}$ is a left adjoint and then use [HAlg, 3.2.3.1]), so does Forget $_{A}$. We deduce using the Barr-Beck theorem (see [HAlg, 6.2.0.6]) that Forget . $_{\text {is }}$ monadic. $_{\text {. }}$. Every dg-Lie algebra can thus be obtained as a colimit of a simplicial diagram with values in the $(\infty, 1)$-category of free dg-Lie algebras (see [HAlg, 6.2.2.12]). We then deduce the result from what precedes.

Remark 1.2.4. The equivalence $\mathbf{d g M o d}_{A} \simeq \mathcal{P}_{\Sigma}^{\mathrm{st}}\left(\mathbf{d g M o d}_{A}^{\mathrm{f}, \mathrm{ft}, \geqslant 1}\right)$ is given by the Yoneda embedding. It follows that for any $n$, the shift functor $[n]: \mathbf{d g M o d}_{A} \rightarrow \operatorname{dgMod}_{A}$ corresponds to the composition with the left adjoint $[-n]$ to $[n]$

$$
[-n]^{*}: \mathcal{P}_{\Sigma}^{\mathrm{st}}\left(\operatorname{dgMod}_{A}^{\mathrm{f}, \mathrm{ft}, \geqslant 1}\right) \rightarrow \mathcal{P}_{\Sigma}^{\mathrm{st}}\left(\operatorname{dgMod}_{A}^{\mathrm{f}, \mathrm{ft}, \geqslant 1}\right)
$$

As another example, the forgetful functor $\operatorname{dgLie}_{A} \rightarrow \operatorname{dg} \operatorname{Mod}_{A}$ is given by the composition with Free $_{A}$ and the following diagram commutes

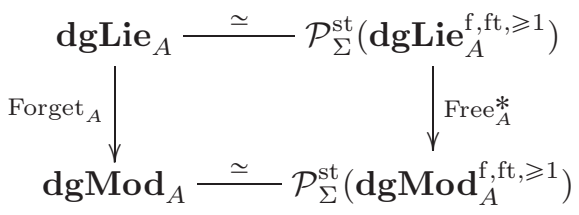


Remark 1.2.5. Whenever $A \rightarrow B$ is a morphism in $\operatorname{cdga}_{k}^{\leqslant 0}$, the following square of $(\infty, 1)$-categories commutes:

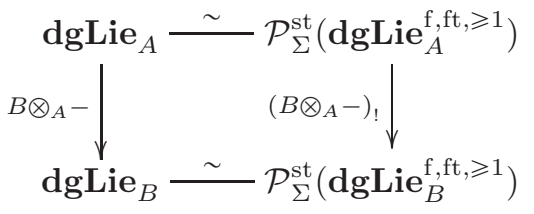

The following proposition actually proves that this comes from a natural transformation between functors cdga $\mathbf{a}_{k}^{\leqslant 0} \rightarrow$ Cat $_{\infty}$.

Proposition 1.2.6. There are $(\infty, 1)$-categories $\int \mathbf{d g L i e}$ and $\int \mathcal{P}_{\Sigma}^{\mathrm{st}}\left(\mathbf{d g L i e} \mathbf{f}^{\mathrm{f}, \mathrm{ft}, \geqslant 1}\right)$, each endowed with a coCartesian fibration to cdga $\leqslant 0$, respectively representing the functors $A \mapsto \mathbf{d g L i e}_{A}$ and $A \mapsto$ $\mathcal{P}_{\Sigma}^{\mathrm{st}}\left(\mathbf{d g L i e}_{A}^{\mathrm{f}, \mathrm{ft}, \geqslant 1}\right)$. There is an equivalence over $\operatorname{cdga}_{k}^{\leq 0}$ :

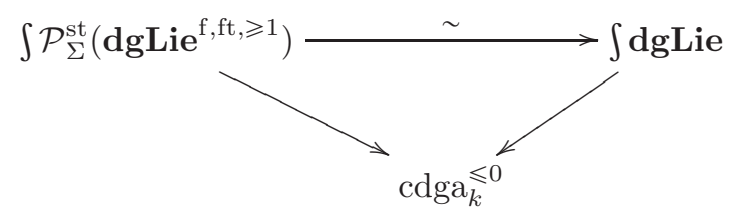

This induces an equivalence of functors $\mathrm{cdga}_{k}^{\leqslant 0} \rightarrow \mathbf{P r}_{\infty}^{\mathrm{L}, \mathbb{U}}$ which moreover descend to a natural transformation

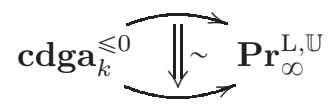

Remark 1.2.7. This proposition establishes an equivalence of functors $\mathbf{c d g a}_{k}^{\leqslant 0} \rightarrow \mathbf{P r}_{\infty}^{\mathrm{L}, \mathbb{U}}$ between $A \mapsto \operatorname{dgLie}_{A}$ and $A \mapsto \mathcal{P}_{\Sigma}^{\text {st }}\left(\mathbf{d g L i e}_{A}^{\mathrm{f}, \mathrm{ft}, \geqslant 1}\right)$.

Proof. Let us define $\int d g L i e$ as the following category.

- An object is couple $(A, L)$ where $A \in \operatorname{cdga}_{k}^{\leqslant 0}$ and $L \in \operatorname{dgLie}_{A}$.

- A morphism $(A, L) \rightarrow\left(B, L^{\prime}\right)$ is a morphism $A \rightarrow B$ and a morphism of $A$-dg-Lie algebras $L \rightarrow L^{\prime}$

It comes with a natural functor $\pi: \int d \operatorname{dgie} \rightarrow \operatorname{cdga}_{k}^{\leqslant 0}$. For any morphism $A \rightarrow B \in \operatorname{cdga}_{k}^{\leqslant 0}$, there is a strict base change functor $-\otimes_{A} B: \operatorname{dgLie}_{A} \rightarrow \mathrm{dgLie}_{B}$, left adjoint to the forgetful functor. It follows that $\pi$ is a coCartesian fibration. Let us call a quasi-isomorphism in $\int \operatorname{dgLie}$ any map $(A, L) \rightarrow\left(B, L^{\prime}\right)$ of which the underlying map $A \rightarrow B$ is an identity and the map $L \rightarrow L^{\prime}$ is a quasi-isomorphism. We define $\int \mathbf{d g L i e}$ to be the $(\infty, 1)$-categorical localization of $\int \mathrm{dgLie}$ along quasi-isomorphisms. Using [DAG-X, 2.4.19], we get a coCartesian fibration of $(\infty, 1)$-categories $p: \int \mathbf{d g L i e} \rightarrow \operatorname{cdga}_{k} \leqslant 0$.

This coCartesian fibration $p$ defines a functor $\mathbf{d g L i e}: \operatorname{cdga}_{k}^{\leqslant 0} \rightarrow \mathbf{C a t}_{\infty}^{\mathbb{V}}$ mapping a cdga $A$ to $\mathbf{d g L i e}_{A}$ and a morphism $A \rightarrow B$ to the corresponding (derived) base change functor. It comes with a subfunctor

$$
\operatorname{dgLie}^{\mathrm{f}, \mathrm{ft}, \geqslant 1}: \operatorname{cdga}_{k}^{\leqslant 0} \rightarrow \mathbf{C a t}_{\infty}^{\mathbb{U}}
$$

Let us denote by $\mathcal{P}_{\Sigma}^{\mathrm{st}}\left(\mathbf{d g}_{\mathbf{L}} \mathbf{L i e} \mathrm{e}^{\mathrm{f}, \mathrm{ft}, \geqslant 1}\right)$ its composite functor with

$$
\mathcal{P}_{\Sigma}^{\mathrm{st}}: \mathbf{C a t}_{\infty}^{\mathbb{U}} \rightarrow \mathbf{C a t}_{\infty}^{\mathbb{V}}
$$

Let us denote by $\int \mathbf{d g L i e} \mathbf{f}^{\mathrm{f}, \mathrm{ft}, \geqslant 1} \rightarrow \operatorname{cdga}_{k}^{\leqslant 0}$ the coCartesian fibration given by the functor $\mathbf{d g L i e}^{\mathrm{f}, \mathrm{ft}, \geqslant 1}$ and by $\int \mathcal{P}_{\Sigma}^{\text {st }}\left(\mathbf{d g L i e} \mathbf{e}^{\mathrm{f}, \mathrm{ft}, \geqslant 1}\right)$ that classified by $\mathcal{P}_{\Sigma}^{\mathrm{st}}\left(\mathbf{d g L i e} \mathbf{f}^{\mathrm{f}, \mathrm{ft}, \geqslant 1}\right)$. 
We get a diagram

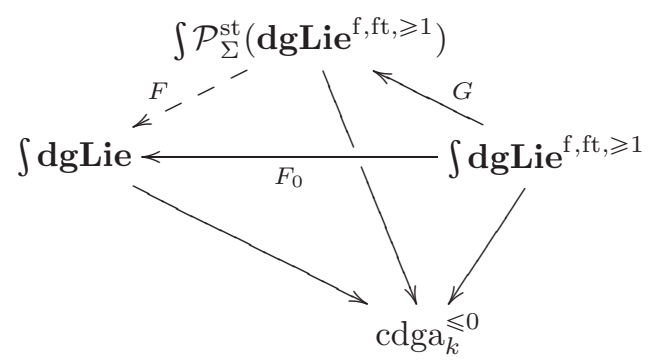

The functor $F_{0}$ has a relative left Kan extension $F$ along $G$ (see [HTT, 4.3.2.14]). From proposition 1.2.2 we get that $F$ is a fibrewise equivalence. It now suffices to prove that $F$ preserves coCartesian morphisms. This is a consequence of remark 1.2.5. We get the announced equivalence of functors

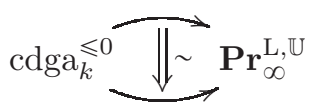

We now observe that both the involved functors map quasi-isomorphisms of $\operatorname{cdga}_{k}^{\leqslant 0}$ to equivalences of categories. It follows that this natural transformation factors through the localisation $\mathbf{c d g a}_{k}^{\leqslant 0}$ of $\operatorname{coga}_{k} \leq 0$.

Remark 1.2.8. In the above proof, we used a general method we will use again later on. Starting with a coCartesian fibration of strict categories $C \rightarrow D$, we want to localise it along some classes of weak equivalences $W$ and $W^{\prime}$. The goal is to get a relevant functor

$$
\mathcal{D}=D\left[W^{\prime}\right] \rightarrow \mathbf{C a t}_{\infty}^{\mathbb{V}}
$$

under the right assumptions. For any object $d \in D$, assume we are given a set of weak equivalences $W_{d}$ in the fibre $C_{d}$ such that for any $d \rightarrow d^{\prime}$, the induced functor $C_{d} \rightarrow C_{d^{\prime}}$ preserves weak equivalences. Using [DAG-X, 2.4.19], we localise $C$ along $W=\bigcup W_{d}$ and get a coCartesian fibration of $(\infty, 1)$ categories

$$
\mathcal{C}=C\left[W^{-1}\right] \rightarrow D
$$

This fibration is classified by a functor $F: D \rightarrow \mathbf{C a t}_{\infty}^{\mathbb{V}}$, mapping $d \in D$ to $\mathcal{C}_{d} \simeq C_{d}\left[W_{d}^{-1}\right]$. If $D$ has a class of weak equivalence $W^{\prime}$ and if $F$ maps morphisms in $W^{\prime}$ to equivalences of $(\infty, 1)$-categories, we get the announced functor $\mathcal{D}=D\left[W^{\prime}\right] \rightarrow \mathbf{C a t}_{\infty}^{\mathbb{V}}$ This procedure will be used extensively in the second part of this work.

\subsection{Almost finite cellular objects}

Let $A$ be a commutative dg-algebra over $k$.

Definition 1.3.1. Let $M$ be an $A$-dg-module.

- We will denote by $M^{\mathrm{c}}(M)$ the mapping cone of the identity of $M$.

- We will say that $M$ is an almost finite cellular object if there is a diagram

$$
0 \rightarrow A^{p_{0}}=M_{0} \rightarrow M_{1} \rightarrow \ldots
$$

whose colimit is $M$ and such that for any $n$, the morphism $M_{n} \rightarrow M_{n+1}$ fits into a cocartesian diagram

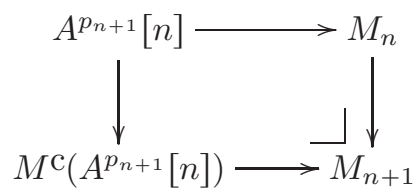


Remark 1.3.2. We choose here to use an explicit model, so that any almost finite cellular object is cofibrant (see lemma below). This will allow us to compute explicitly the dual of an almost finite cellular object (see the proof of lemma 1.4.12). The definition above states that a dg-module $M$ is an almost finite cellular object if it is obtained from 0 by gluing a finite number of cells in each degree (although the total number of cells is not necessarily finite).

Lemma 1.3.3. Let $\phi: M \rightarrow N$ be a morphism of $A$-dg-modules.

- If $M$ is an almost finite cellular object then it is cofibrant.

- Assume both $M$ and $N$ are almost finite cellular objects. The morphism $\phi$ is a quasi-isomorphism if and only if for any field $l$ and any morphism $A \rightarrow l$ the induced map $\phi_{k}: M \otimes_{A} l \rightarrow N \otimes_{A} l$ is a quasi-isomorphism.

Remark 1.3.4. The second point in the above lemma is an analogue to the usual Nakayama lemma.

Proof. Assume $M$ is an almost finite cellular object. Let us consider a diagram $M \rightarrow Q \leftarrow P$ where the map $P \rightarrow Q$ is a trivial fibration. Each morphism $M_{n} \rightarrow M_{n+1}$ is a cofibration and there thus is a compatible family of lifts $\left(M_{n} \rightarrow P\right)$. This gives us a lift $M \rightarrow P$. The $A$-dg-module $M$ is cofibrant.

Let now $\phi$ be a morphism $M \rightarrow N$ between almost finite cellular objects and that the morphism $\phi_{l}$ is a quasi-isomorphism for any field $l$ under $A$. Replacing $M$ with the cone of $\phi$ (which is also an almost finite cellular object) we may assume that $N$ is trivial. Notice first that an almost finite cellular object is concentrated in non positive degree. Notice also that for any $n$ the truncation morphism $\alpha^{\geqslant-n}: M_{n+1}^{\geqslant-n} \rightarrow M^{\geqslant-n}$ is a quasi-isomorphism. We then have

$$
0 \simeq \mathrm{H}^{j}(\underset{A}{M \underset{A}{\otimes} l}) \simeq \mathrm{H}^{j}\left(M_{n} \underset{A}{\otimes} l\right)
$$

whenever $-n<j \leqslant 0$ and for any $A \rightarrow l$. Since $\mathrm{H}^{j}\left(M_{n} \otimes_{A} l\right) \simeq 0$ if $j \leqslant-n-2$ the $A$-dg-module $M_{n}$ is perfect and of amplitude $[-n-1,-n]$. This implies the existence of two projective modules $P$ and $Q$ (ie retracts of some power of $A$ ) fitting in a cofibre sequence (see [TV])

$$
P[n] \rightarrow M_{n} \rightarrow Q[n+1]
$$

The dg-module $M_{n}$ is then cohomologically concentrated in degree ] $\left.-\infty,-n\right]$, and so is $M$. This being true for any $n$ we deduce that $M$ is contractible.

The next lemma requires the base $A \in \mathbf{c d g a}_{k}^{\leqslant 0}$ to be noetherian. Recall that $A$ is noetherian if $\mathrm{H}^{0}(A)$ is noetherian and if $\mathrm{H}^{-n}(A)$ is trivial when $n$ is big enough and of finite type over $\mathrm{H}^{0}(A)$ for any $n$. Note that since $A \in \mathbf{c d g a}_{k}^{\leq 0}$, we always have $\mathrm{H}^{n}(A)=0$ for $n>0$.

Lemma 1.3.5. Assume $A$ is noetherian. If $B$ is an object of $\operatorname{cdga}_{A}^{\leqslant 0} / A$ such that:

- The $\mathrm{H}^{0}(A)$-algebra $\mathrm{H}^{0}(B)$ is finitely presented,

- For any $n \geqslant 1$ the $\mathrm{H}^{0}(B)$-module $\mathrm{H}^{-n}(B)$ is of finite type,

then the $A$-dg-module $\mathbb{L}_{B / A} \otimes_{B} A$ is an almost finite cellular object.

Remark 1.3.6. The lemma above is closely related to [HAlg, 8.4.3.18].

Proof. Because the functor $(A \rightarrow B \rightarrow A) \mapsto \mathbb{L}_{B / A} \otimes_{B} A$ preserves colimits, it suffices to prove that $B$ is an almost finite cellular object in $\operatorname{cdga}_{A}^{\leqslant 0} / A$. This means we have to build a diagram

$$
B_{0} \rightarrow B_{1} \rightarrow \ldots
$$


whose colimit is equivalent to $B$ and such that for any $n \geqslant 1$ the morphism $B_{n-1} \rightarrow B_{n}$ fits into a cocartesian diagram

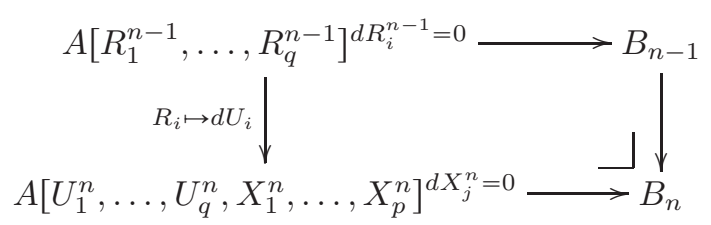

where $R_{i}^{n-1}$ is a variable in degree $-(n-1)$ and $X_{j}^{n}$ and $U_{i}^{n}$ are variables in degree $-n$.

We build such a diagram recursively. Let

$$
\mathrm{H}^{0}(B) \cong \mathrm{H}^{0}(A)\left[X_{1}^{0}, \ldots, X_{p_{0}}^{0}\right] /\left(R_{1}^{0}, \ldots, R_{q_{0}}^{0}\right)
$$

be a presentation of $\mathrm{H}^{0}(B)$ as a $\mathrm{H}^{0}(A)$-algebra. Let $B_{0}$ be $A\left[X_{1}^{0}, \ldots, X_{p_{0}}^{0}\right]$ equipped with a morphism $\phi_{0}: B_{0} \rightarrow B$ given by a choice of coset representatives of $X_{1}^{0}, \ldots, X_{p_{0}}^{0}$ in $B$. The induced morphism $\mathrm{H}^{0}\left(B_{0}\right) \rightarrow \mathrm{H}^{0}(B)$ is surjective and its kernel is of finite type (as a $\mathrm{H}^{0}(A)$-module).

Let $n \geqslant 1$. Assume $\phi_{n-1}: B_{n-1} \rightarrow B$ has been defined and satisfies the properties:

- If $n=1$ then the induced morphism of $\mathrm{H}^{0}(A)$-modules $\mathrm{H}^{0}\left(B_{0}\right) \rightarrow \mathrm{H}^{0}(B)$ is surjective and its kernel $K_{0}$ is a $\mathrm{H}^{0}(A)$-module of finite type.

- If $n \geqslant 2$, then the morphism $\phi_{n-1}$ induces isomorphisms $\mathrm{H}^{-i}\left(B_{n-1}\right) \rightarrow \mathrm{H}^{-i}(B)$ of $\mathrm{H}^{0}(A)$ modules if $i=0$ and of $\mathrm{H}^{0}(B)$-modules for $1 \leqslant i \leqslant n-2$.

- If $n \geqslant 2$ then the induced morphism of $\mathrm{H}^{0}(B)$-modules $\mathrm{H}^{-n+1}\left(B_{n-1}\right) \rightarrow \mathrm{H}^{-n+1}(B)$ is surjective and its kernel $K_{n-1}$ is a $\mathrm{H}^{0}(B)$-module of finite type.

Let $n \geqslant 1$. Let $X_{1}^{n}, \ldots, X_{p}^{n}$ be generators of $\mathrm{H}^{-n}(B)$ as a $\mathrm{H}^{0}(B)$-module and $R_{1}^{n-1}, \ldots, R_{q}^{n-1}$ be generators of $K_{n-1}$. Let $B_{n}$ be the pushout:

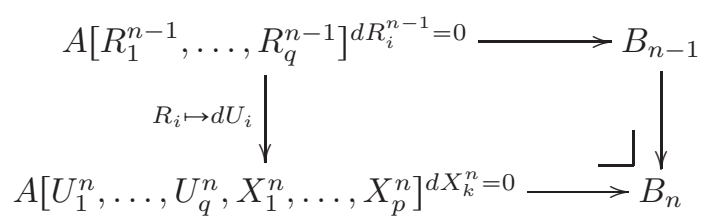

Let $r_{1}^{n-1}, \ldots, r_{q}^{n-1}$ be the images of $R_{1}^{n-1}, \ldots, R_{q}^{n-1}$ (respectively) by the composite morphism

$$
A\left[R_{1}^{n-1}, \ldots, R_{q}^{n-1}\right]^{d R_{i}^{n-1}=0} \rightarrow B_{n-1} \rightarrow B
$$

There exist $u_{1}^{n}, \ldots, u_{q}^{n} \in B$ such that $d u_{i}^{n}=r_{i}^{n-1}$ for all $i$. Those $u_{1}^{n}, \ldots, u_{q}^{n}$ together with a choice of coset representatives of $X_{1}^{n}, \ldots, X_{p}^{n}$ in $B$ induce a morphism

$$
A\left[U_{1}^{n}, \ldots, U_{q}^{n}, X_{1}^{n}, \ldots, X_{p}^{n}\right]^{d X_{k}^{n}=0} \rightarrow B
$$

which induces a morphism $\phi_{n}: B_{n} \rightarrow B$.

If $n=1$ then a quick computation proves the isomorphism of $\mathrm{H}^{0}(A)$-modules

$$
\mathrm{H}^{0}\left(B_{1}\right) \cong \mathrm{H}^{0}\left(B_{0}\right) /\left(R_{1}^{0}, \ldots, R_{q}^{0}\right) \cong \mathrm{H}^{0}(B)
$$

If $n \geqslant 2$ then the truncated morphism $B_{n}^{\geqslant 2-n} \stackrel{\sim}{\rightarrow} \mathrm{B}_{n-1}^{\geqslant 2-n}$ is a quasi-isomorphism and the induced morphisms $\mathrm{H}^{-i}\left(B_{n}\right) \stackrel{\simeq}{\rightarrow} \mathrm{H}^{-i}(B)$ are thus isomorphisms of $\mathrm{H}^{0}(B)$-modules for $i \leqslant n-2$. We then get the isomorphism of $\mathrm{H}^{0}(B)$-modules

$$
\mathrm{H}^{-n+1}\left(B_{n}\right) \cong \mathrm{H}^{-n+1}\left(B_{n-1}\right) /\left(R_{1}^{n-1}, \ldots, R_{q}^{n-1}\right) \cong \mathrm{H}^{-n+1}(B)
$$


The natural morphism $\theta: \mathrm{H}^{-n}\left(B_{n}\right) \rightarrow \mathrm{H}^{-n}(B)$ is surjective. The $\mathrm{H}^{0}(B)$-module $\mathrm{H}^{-n}\left(B_{n}\right)$ is of finite type and because $\mathrm{H}^{0}(B)$ is noetherian, the kernel $K_{n}$ of $\theta$ is also of finite type. The recursivity is proven and it now follows that the morphism $\operatorname{colim}_{n} B_{n} \rightarrow B$ is a quasi-isomorphism.

Definition 1.3.7. Let $L$ be a dg-Lie algebra over $A$.

- We will say that $L$ is very good if there exists a finite sequence

$$
0=L_{0} \rightarrow L_{1} \rightarrow \ldots \rightarrow L_{n}=L
$$

such that each morphism $L_{i} \rightarrow L_{i+1}$ fits into a cocartesian square

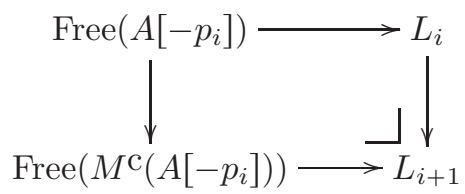

where $p_{i} \geqslant 2$.

- We will say that $L$ is good if it is quasi-isomorphic to a very good dg-Lie algebra.

- We will say that $L$ is almost finite if it is cofibrant and if its underlying graded module is isomorphic to

$$
\bigoplus_{i \geqslant 1} A^{n_{i}}[-i]
$$

Remark 1.3.8. The notions of almost finite dg-Lie algebras and of almost finite cellular objects are closely related. We will see in the proof of lemma 1.4.12 that the dual $L^{\vee}$ of an almost finite dg-Lie algebra is an almost finite cellular dg-module.

Lemma 1.3.9. The following assertions are true.

- Any very good dg-Lie algebra is almost finite.

- The underlying dg-module of a cofibrant dg-Lie algebra is cofibrant.

Proof. Any free dg-Lie algebra generated by some $A[-p]$ with $p \geqslant 2$ is almost finite (it is actually obtained by base change from an almost finite dg-Lie algebra on $k$ ). Considering a pushout diagram

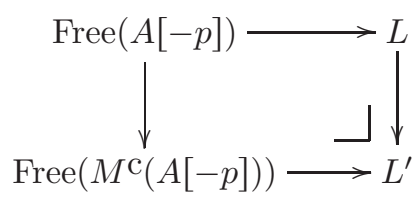

Whenever $L$ is almost finite, so is $L^{\prime}$. This proves the first item.

Let now $L$ be a dg-Lie algebra over $A$. There is a morphism of dg-modules $L \rightarrow \mathcal{U}_{A} L$. The Poincaré-Birkhoff-Witt theorem states that the dg-module $\mathcal{U}_{A} L$ is isomorphic to $\operatorname{Sym}_{A} L$. There is therefore a retract $\mathcal{U}_{A} L \rightarrow L$ of the universal morphism $L \rightarrow \mathcal{U}_{A} L$. The functor $\mathcal{U}_{A}: \operatorname{dgLie} \operatorname{Lig}_{A} \rightarrow \operatorname{dg} \operatorname{Alg}_{A}$ preserves cofibrant objects and using a result of [SS], so does the forgetful functor $\operatorname{dg} \operatorname{Alg}_{A} \rightarrow \operatorname{dgMod}_{A}$. We therefore deduce that if $L$ is cofibrant in $\operatorname{dgLie}_{A}$ it is also cofibrant in $\operatorname{dg} \operatorname{Mod}_{A}$.

Definition 1.3.10. Let $\mathbf{d g L i e}{ }_{A}^{\text {good }}$ denote the sub- $(\infty, 1)$-category of $\mathbf{d g L i e}{ }_{A}$ spanned by good dg-Lie algebras.

Remark 1.3.11. We naturally have an inclusion $\mathbf{d g L i e}{ }_{A}^{\mathrm{f}, \mathrm{ft}, \geqslant 1} \rightarrow \mathbf{d g L i e} \mathbf{A}_{A}^{\operatorname{good}}$. 


\subsection{Homology and cohomology of dg-Lie algebras}

The content of this section can be found in [DAG-X] when the base is a field. Proofs are simple avatars of Lurie's on a more general base $A$. Let then $A$ be a commutative dg-algebra concentrated in non-positive degree over a field $k$ of characteristic zero.

Definition 1.4.1. Let $A[\eta]$ denote the (contractible) commutative $A$-dg-algebra generated by one element $\eta$ of degree -1 such that $\eta^{2}=0$ and $d \eta=1$. For any $A$-dg-Lie algebra $L$, the tensor product $A[\eta] \otimes_{A} L$ is still an $A$-dg-Lie algebra and we can thus define the homological Chevalley-Eilenberg complex of $L$ :

$$
\mathrm{H}_{A}(L)=\mathcal{U}_{A}(A[\eta] \underset{A}{\otimes} L) \underset{\mathcal{U}_{A} L}{\otimes} A
$$

where $\mathcal{U}_{A}: \operatorname{dgLie}_{A} \rightarrow \operatorname{dgAlg}_{A}$ is the functor sending a Lie algebra to its enveloping algebra. This construction defines a strict functor:

$$
\mathrm{H}_{A}: \operatorname{dgLie}_{A} \rightarrow A / \operatorname{dg} \operatorname{Mod}_{A}
$$

Remark 1.4.2. The complex $\mathrm{H}_{A}(L)$ is isomorphic as a graded module to $\operatorname{Sym}_{A}(L[1])$, the symmetric algebra built on $L[1]$. The differentials do not coincide though. The one on $\mathrm{H}_{A}(L)$ is given on homogenous objects by the following formula:

$$
\begin{aligned}
d\left(\eta \cdot x_{1} \otimes \ldots \otimes \eta \cdot x_{n}\right)=\sum_{i<j}(-1)^{T_{i j}} \eta \cdot\left[x_{i}, x_{j}\right] \otimes & \eta \cdot x_{1} \otimes \ldots \otimes \widehat{\eta \cdot x_{i}} \otimes \ldots \otimes \widehat{\eta \cdot x_{j}} \otimes \ldots \otimes \eta \cdot x_{n} \\
& -\sum_{i}(-1)^{S_{i}} \eta \cdot x_{1} \otimes \ldots \otimes \eta \cdot d\left(x_{i}\right) \otimes \ldots \otimes \eta \cdot x_{n}
\end{aligned}
$$

where $\eta . x$ denotes the point in $L[1]$ corresponding to $x \in L$.

$$
\begin{aligned}
& S_{i}=i-1+\left|x_{1}\right|+\cdots+\left|x_{i-1}\right| \\
& T_{i j}=\left(\left|x_{i}\right|-1\right) S_{i}+\left(\left|x_{j}\right|-1\right) S_{j}+\left(\left|x_{i}\right|-1\right)\left(\left|x_{j}\right|-1\right)
\end{aligned}
$$

The coalgebra structure on $\operatorname{Sym}_{A}(L[1])$ is compatible with this differential and the isomorphism above induces a coalgebra structure on $\mathrm{H}_{A}(L)$ given for $x \in L$ homogenous by:

$$
\Delta(\eta . x)=\eta . x \otimes 1+1 \otimes \eta . x
$$

Proposition 1.4.3. The functor $\mathrm{H}_{A}$ preserves quasi-isomorphisms. It induces a functor between the corresponding $(\infty, 1)$-categories, which we will denote the same way:

$$
\mathrm{H}_{A}: \operatorname{dgLie}_{A} \rightarrow A / \operatorname{dgMod}{ }_{A}
$$

Proof. Let $L \rightarrow L^{\prime}$ be a quasi-isomorphism of $A$-dg-Lie algebras. Both $\mathrm{H}_{A}(L)$ and $\mathrm{H}_{A}\left(L^{\prime}\right)$ are endowed with a natural filtration denoted $\mathrm{H}_{A}^{\leqslant n}(L)$ ( $\operatorname{resp} L^{\prime}$ ) induced by the canonical filtration of $\operatorname{Sym}_{A}(L[1])$. Because quasi-isomorphisms are stable by filtered colimits, it is enough to prove that each morphism $\mathrm{H}_{A}^{\leqslant n}(L) \rightarrow \mathrm{H}_{A}^{\leqslant n}\left(L^{\prime}\right)$ is a quasi-isomorphism. The case $n=0$ is trivial. Let us assume $\mathrm{H}_{A}^{\leqslant n-1}(L) \rightarrow \mathrm{H}_{A}^{\leqslant n-1}\left(L^{\prime}\right)$ to be a quasi-isomorphism. There are short exact sequences:

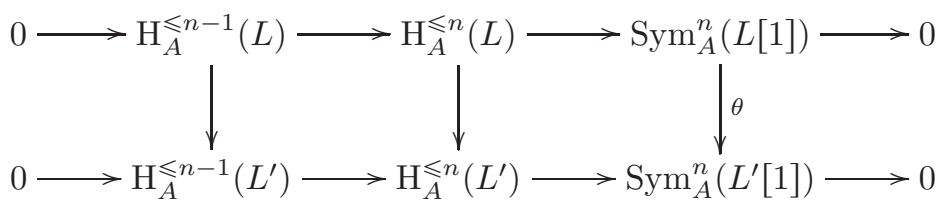

The base dg-algebra $A$ is of characteristic zero and the morphism $\theta$ is thus a retract of the quasiisomorphism $L[1]^{\otimes n} \rightarrow L^{\prime}[1]^{\otimes n}$ (where the tensor product is taken over $A$ ). 
Proposition 1.4.4. Let $A \rightarrow B$ be a morphism in $\mathbf{c d g a}_{k}^{\leqslant 0}$. The following square is commutative:

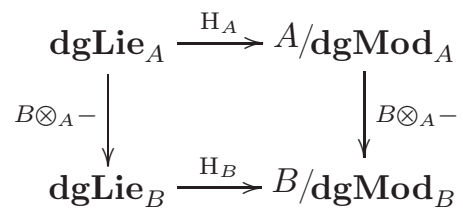

Proof. This follows directly from the definition.

Corollary 1.4.5. Let $L$ be in $\mathrm{dgLie}_{A}$ freely generated by some free dg-module $M$. The homological Chevalley-Eilenberg complex $\mathrm{H}_{A}(L)$ of $L$ is quasi-isomorphic to the pointed dg-module $A \rightarrow A \oplus M[1]$.

Proof. This a consequence of the previous proposition and the corresponding result over a field in Lurie's theorem 1.0.1.

Definition 1.4.6. Let $L$ be an object of $\operatorname{dgLie}_{A}$. We define the cohomological Chevalley-Eilenberg complex of $L$ as the dual of its homological:

$$
\mathrm{C}_{A}(L)=\mathrm{H}_{A}(L)^{\vee}=\underline{\operatorname{Hom}}_{A}\left(\mathrm{H}_{A}(L), A\right)
$$

It is equipped with a commutative algebra structure (see remark 1.4.2). This defines a functor:

$$
\mathrm{C}_{A}: \operatorname{dgLie}_{A} \rightarrow\left(\operatorname{cdga}_{A} / A\right)^{\mathrm{op}}
$$

between $(\infty, 1)$-categories.

Remark 1.4.7. The Chevalley-Eilenberg cohomology of an object $L$ of $\mathbf{d g L i e} \mathbf{A}_{A}^{\mathrm{f}, \mathrm{ft}, \geqslant 1}$ is concentrated in non positive degree. It indeed suffices to dualise the quasi-isomorphism from corollary 1.4.5. The following proposition proves the cohomology of a good dg-Lie algebra is also concentrated in nonpositive degree.

Proposition 1.4.8. The functor $\mathrm{C}_{A}$ of $(\infty, 1)$-categories maps colimit diagrams in $\mathbf{d g L i e}{ }_{A}$ to limit diagrams of $\operatorname{cdga}_{A} / A$.

Proof (sketch of a). For a complete proof, the author refers to the proof of proposition 2.2.12 in [DAG-X]. We will only transcript here the main arguments.

A commutative $A$-dg-algebra $B$ is the limit of a diagram $B_{\alpha}$ if and only if the underlying dgmodule is the limit of the underlying diagram of dg-modules. It is thus enough to consider the composite $\infty$-functor $\mathbf{d g L i e}_{A} \rightarrow\left(\operatorname{cdga}_{A} / A\right)^{\mathrm{op}} \rightarrow\left(\operatorname{dgMod}_{A} / A\right)^{\mathrm{op}}$. This functor is equivalent to $\left(\mathrm{H}_{A}(-)\right)^{\vee}$. It is then enough to prove $\mathrm{H}_{A}: \mathbf{d g L i e}_{A} \rightarrow A / \mathbf{d g M o d}_{A}$ to preserve colimits.

To do so, we will first focus on the case of sifted colimits, which need only to be preserved by the composite functor $\mathbf{d g L i e}_{A} \rightarrow A / \mathbf{d g M o d}{ }_{A} \rightarrow \mathbf{d g M o d}{ }_{A}$. This last functor is the (filtered) colimits of the functors $\mathrm{H}_{A}^{\leqslant n}$ as introduced in the proof of proposition 1.4.3. We now have to prove that $\mathrm{H}_{A}^{\leqslant n}: \mathbf{d g L i e}_{A} \rightarrow \mathbf{d g M o d}{ }_{A}$ preserves sifted colimits, for any $n$. There is a fiber sequence

$$
\mathrm{H}_{A}^{\leqslant n-1} \rightarrow \mathrm{H}_{A}^{\leqslant n} \rightarrow \operatorname{Sym}_{A}^{n}((-)[1])
$$

The functor $\operatorname{Sym}_{A}((-)[1])$ preserves sifted colimits in characteristic zero and an inductive process proves that $\mathrm{H}_{A}$ preserves sifted colimits too.

We now have to treat the case of finite coproducts. The initial object is obviously preserved. Let $L=L^{\prime} \amalg L^{\prime \prime}$ be a coproduct of dg-Lie algebras. We proved in remark 1.2.3 that $L^{\prime}$ an $L^{\prime \prime}$ can be written as sifted colimits of objects of $\mathbf{d g L i e}_{A}^{\mathrm{f}, \mathrm{ft}, \geqslant 1}$. It is thus enough to prove that $\mathrm{H}_{A}$ preserve the 
coproduct $L=L^{\prime} \amalg L^{\prime \prime}$ when $L^{\prime}$ and $L^{\prime \prime}$ (and thus $L$ too) are in $\mathbf{d g L i e}_{A}^{\mathrm{f}, \mathrm{ft}, \geqslant 1}$. This corresponds to the following cocartesian diagram

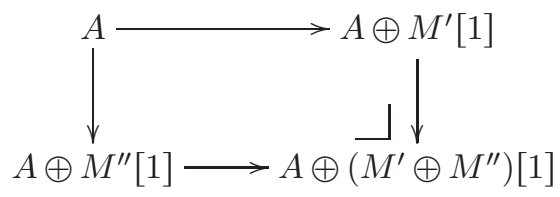

where $M^{\prime}$ and $M^{\prime \prime}$ are objects of $\mathbf{d g M o d}_{A}^{\mathrm{f}, \mathrm{ft}, \geqslant 1}$ generating $L^{\prime}$ and $L^{\prime \prime}$ respectively.

Definition 1.4.9. The colimit-preserving functor $\mathrm{C}_{A}$ between presentable $(\infty, 1)$-categories admits a right adjoint which we will denote by $\mathrm{D}_{A}$.

Lemma 1.4.10. Let $B \rightarrow A$ be a morphism of $\mathbf{c d g a}_{k}^{\leqslant 0}$. The following diagram of $(\infty, 1)$-categories commutes:

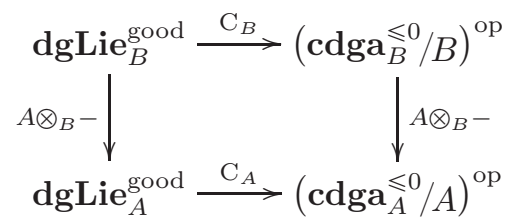

Proof. The proposition 1.4.4 gives birth to a natural transformation $A \otimes_{B} \mathrm{C}_{B}(-) \rightarrow \mathrm{C}_{A}\left(A \otimes_{B}-\right)$. Let $L \in \mathbf{d g L i e}_{B}^{\text {good }}$. The $B$-dg-module $\mathrm{C}_{A}\left(A \otimes_{B} L\right)$ is equivalent to

$$
\mathrm{C}_{A}(A \underset{B}{\otimes} L) \simeq \underline{\operatorname{Hom}}_{B}\left(\mathrm{H}_{B}(L), A\right)
$$

We thus study the natural morphism

$$
\phi_{L}: A \underset{B}{\otimes} \mathrm{C}_{B}(L) \rightarrow \underline{\operatorname{Hom}}_{B}\left(\mathrm{H}_{B}(L), A\right)
$$

Let us consider the case of the free dg-Lie algebra $L=\operatorname{Free}(B[-p])$ with $p \geqslant 1$. If $B$ is the base field $k$ then $\mathrm{H}_{k}(L)$ is perfect (corollary 1.4.5) and the morphism $\phi_{L}$ is an equivalence. If $B$ is any $k$-dg-algebra then $L$ is equivalent to $B \otimes_{k} \operatorname{Free}(k[-p])$ and we conclude using proposition 1.4.4 that $\phi_{L}$ is an equivalence.

To prove the general case of any good dg-Lie algebra it is now enough to ensure that if $L_{1} \leftarrow$ $L_{0} \rightarrow L_{2}$ is a diagram of good dg-Lie algebras such that $\phi_{L_{1}}, \phi_{L_{0}}$ and $\phi_{L_{2}}$ are equivalences then so is $\phi_{L}$, with $L=L_{1} \amalg_{L_{0}} L_{2}$. Using proposition 1.4.8, we see it can be tested in $\operatorname{dgMod}_{A}$ in which tensor product and fibre product commute.

Corollary 1.4.11. The composite functor $\mathrm{C}_{A}$ Free $_{A}: \operatorname{dgMod}_{A} \rightarrow \mathbf{d g L i e}_{A} \rightarrow\left(\mathbf{c d g a}_{A} / A\right)^{\mathrm{op}}$ is equivalent to the functor $M \rightarrow A \oplus M^{\vee}[-1]$.

Proof. The $(\infty, 1)$-category $\operatorname{dgMod}_{A}$ is generated under (sifted) colimits by $\operatorname{dgMod}_{A}^{\mathrm{f}, \mathrm{ft}, \geqslant 1}$. The functors at hand coincide on $\operatorname{dgMod}_{A}^{f, f t, \geqslant 1}$ and both preserve colimits.

Lemma 1.4.12. Assume $A$ is noetherian. Let $L$ be a good dg-Lie algebra over $A$. Recall $\mathrm{D}_{A}$ from definition 1.4.9. The adjunction unit $L \rightarrow \mathrm{D}_{A} \mathrm{C}_{A} L$ is a quasi-isomorphism.

Proof. Let us first proves that the morphism at hand is equivalent, as a morphism of dg-modules, to the natural morphism $L \rightarrow L^{\vee v}$. The composite functor Forget ${ }_{A} \mathrm{D}_{A}:\left(\mathbf{c d g a}_{A} / A\right)^{\mathrm{op}} \rightarrow \operatorname{dgLie}_{A} \rightarrow$ $\operatorname{dgMod}_{A}$ is right adjoint to $\mathrm{C}_{A}$ Free $_{A}$. Using corollary 1.4.11, we see that Forget ${ }_{A} \mathrm{D}_{A}$ is right adjoint to the composite functor

$$
\operatorname{dgMod}_{A} \stackrel{(-)^{\vee}[-1]}{\longrightarrow} \operatorname{dgMod}_{A}^{\mathrm{op}} \stackrel{A \oplus-}{\longrightarrow}\left(\operatorname{cdga}_{A} / A\right)^{\mathrm{op}}
$$


The functor $(-)^{\vee}[-1]$ admits a right adjoint, namely $(-[1])^{\vee}$ while $A \oplus-$ is left adjoint (beware of the op's) to

$$
(A \rightarrow B \rightarrow A) \mapsto A \underset{B}{\otimes} \mathbb{L}_{B / A}
$$

It follows that Forget ${ }_{A} \mathrm{D}_{A}$ is equivalent to the functor :

$$
(A \rightarrow B \rightarrow A) \mapsto\left(A \underset{B}{\otimes} \mathbb{L}_{B / A}[1]\right)^{\vee}
$$

The adjunction unit $L \rightarrow \mathrm{D}_{A} \mathrm{C}_{A} L$ is thus dual to a map

$$
f: \mathbb{L}_{\mathrm{C}_{A} L / A} \underset{\mathrm{C}_{A} L}{\otimes} A \rightarrow L^{\vee}[-1]
$$

As soon as $L$ is good and $A$ noetherian, the complex $\mathrm{C}_{A} L$ satisfies the finiteness conditions of lemma 1.3.5. We can safely assume that $L$ is very good. Because $L$ is almost finite (as a dg-Lie algebra), there is a family $\left(n_{i}\right)$ of integers and an isomorphism of graded modules

$$
L=\bigoplus_{i \geqslant 1} A^{n_{i}}[-i]
$$

The dual $L^{\vee}$ of $L$ can be computed naively (since the underlying dg-module of $L$ is cofibrant). The dual $L^{\vee}$ is then isomorphic to $\prod_{i \geqslant 1} A^{n_{i}}[i]$ with an extra differential. Because $A$ in concentrated in non positive degree, only a finite number of terms contribute to a fixed degree in this product. The dual $L^{\vee}$ is hence equivalent to $\bigoplus_{i \geqslant 1} A^{n_{i}}[i]$ (with the extra differential). The dg-module $L^{\vee}[-1]$ is hence an almost finite cellular object. Both domain and codomain of the morphism $f$ are thus almost finite cellular $A$-dg-modules. It is then enough to consider $f \otimes_{A} l$ for any field $l$ and any morphism $A \rightarrow l$

$$
f \underset{A}{\otimes} l:\left(\mathbb{L}_{\mathrm{C}_{A}(L) / A} \otimes A\right) \underset{A}{\otimes} l \rightarrow(L \otimes l)^{\vee}[-1]
$$

The lemma 1.4.10 gives us the equivalence $\mathrm{C}_{A}(L) \otimes_{A} l \simeq \mathrm{C}_{l}\left(L \otimes_{A} l\right)$ and the morphism $f \otimes_{A} l$ is thus equivalent to the morphism

$$
\mathbb{L}_{\mathrm{C}_{l}\left(L \otimes_{A} l\right) / l} \otimes l \rightarrow(L \otimes l)^{\vee}[-1]
$$

This case is equivalent to Lurie's result 1.0.1 (ii). We get that $f$ is an equivalence and that the adjunction morphism $L \rightarrow \mathrm{D}_{A} \mathrm{C}_{A} L$ is equivalent to the canonical map $L \rightarrow L^{\vee}$.

We now prove that $L \rightarrow L^{\vee \vee}$ is an equivalence. We saw above the equivalence $L^{\vee} \simeq \bigoplus_{i \geqslant 1} A^{n_{i}}[i]$. The natural morphism $L \rightarrow L^{\vee} \vee$ therefore corresponds to the morphism

$$
\bigoplus_{i \geqslant 1} A^{n_{i}}[-i] \rightarrow \prod_{i \geqslant 1} A^{n_{i}}[-i]
$$

Since $A$ is noetherian, it is cohomologically bounded. Once more, only a finite number of terms actually contribute to a fixed degree and the map above is a quasi-isomorphism.

Remark 1.4.13. The base dg-algebra $A$ needs to be cohomologically bounded for that lemma to be true. Taking $\mathrm{H}^{0}(A)$ noetherian and $L=\operatorname{Free}\left(A^{2}[-1]\right)$, the adjunction morphism is equivalent to

$$
L \rightarrow L^{\vee \vee}
$$

which is not a quasi-isomorphism if $A$ is not cohomologically bounded.

\subsection{Formal stack over a dg-algebra}

Throughout this section $A$ will denote an object of $\mathbf{c d g a}_{k}^{\leqslant 0}$.

Definition 1.5.1. Let $\operatorname{dgExt}_{A}$ denote the full sub-category of $\operatorname{cdga}_{A}^{\leqslant 0} / A$ spanned be the trivial square zero extensions $A \oplus M$, where $M$ is a free $A$-dg-module of finite type concentrated in non positive degree. 
Definition 1.5.2. A formal stack over $A$ is a functor $\operatorname{dgExt}_{A} \rightarrow \mathbf{s S e t s}$ preserving finite products and loop spaces. We will denote by $\mathbf{d S t}_{A}^{\mathrm{f}}$ the $(\infty, 1)$-category of such formal stacks:

$$
\mathbf{d S t}_{A}^{\mathrm{f}}=\mathcal{P}_{\Sigma}^{\mathrm{st}}\left(\mathbf{d g E x t} \mathbf{E t}_{A}^{\mathrm{op}}\right)
$$

Remark 1.5.3. The $(\infty, 1)$-category $\mathbf{d S t}_{A}^{\mathrm{f}}$ is $\mathbb{U}$-presentable.

Let $A \in \mathbf{c d g a}_{k}^{\leqslant 0}$. For any formal stack $X$ over $A$ we can consider the functor

$$
\begin{aligned}
& \left(\operatorname{dgMod}_{A}^{\mathrm{f}, \mathrm{ft}, \geqslant 1}\right)^{\mathrm{op}} \rightarrow \quad \text { sSets } \\
& M \quad \mapsto X\left(A \oplus M^{\vee}\right)
\end{aligned}
$$

From $X$ being a formal stack, the functor above belong to $\mathcal{P}_{\Sigma}^{\mathrm{st}}\left(\mathbf{d g M o d}_{A}^{\mathrm{f}, \mathrm{ft}, \geqslant 1}\right)$ and is hence (see proposition 1.2.2) represented by an $A$-dg-module: the tangent of $X$ at its canonical point.

Definition 1.5.4. Let $A \in \mathbf{c d g a}_{k}^{\leqslant 0}$ and let $S=\operatorname{Spec} A$. The tangent complex of a formal stack $X$ over $A$ at the canonical point $x$ is the $A$-dg-module $\mathbb{T}_{X / S, x}$ representing the product-preserving functor

$$
M \mapsto X\left(A \oplus M^{\vee}\right)
$$

Remark 1.5.5. We will link this tangent with the usual tangent of derived Artin stacks in remark 2.2.9.

Proposition 1.5.6. Let $A$ be in $\mathbf{c d g a}_{k}^{\leqslant 0}$ and let $S=\operatorname{Spec} A$. There is an adjunction

$$
\mathcal{F}_{A}: \operatorname{dgLie}_{A} \rightleftarrows \mathrm{dSt}_{A}^{\mathrm{f}}: \mathfrak{L}_{A}
$$

such that

- The functor Forget $\mathfrak{L}_{A}: \mathbf{d S t}_{A}^{\mathrm{f}} \rightarrow \mathbf{d g L i e}_{A} \rightarrow \mathbf{d g M o d}_{A}$ is equivalent to the functor $X \mapsto$ $\mathbb{T}_{X / S, x}[-1]$ where $\mathbb{T}_{X / S, x}$ is the tangent complex of $X$ over $S$ at the natural point $x$ of $X$.

- The functor $\mathfrak{L}_{A}$ is conservative. Its restriction to $\mathbf{d g E x t}_{A}^{\text {op }}$ is canonically equivalent to $\mathrm{D}_{A}$.

- If moreover $A$ is noetherian then the functors $\mathfrak{L}_{A}$ and $\mathcal{F}_{A}$ are equivalences of $(\infty, 1)$-categories.

Definition 1.5.7. Let $X$ be a formal stack over $A$. The Lie algebra $\mathfrak{L}_{A} X$ will be called the tangent Lie algebra of $X$ (over $A$ ).

Proof (of proposition 1.5.6). Let us prove the first item. The functor $\mathrm{C}_{A}$ restricts to a functor

$$
\mathrm{C}_{A}: \operatorname{dgLie}_{A}^{\mathrm{f}, \mathrm{ft}, \geqslant 1} \rightarrow \operatorname{dgExt}_{A}^{\mathrm{op}}
$$

which composed with the Yoneda embedding defines a functor $\phi: \mathbf{d g L i e}_{A}^{\mathrm{f}, \mathrm{ft}, \geqslant 1} \rightarrow \mathbf{d S t}_{A}^{\mathrm{f}}$. This last functor extends by colimits to

$$
\mathcal{F}_{A}: \operatorname{dgLie}_{A} \simeq \mathcal{P}_{\Sigma}^{\mathrm{st}}\left(\operatorname{dgLie}_{A}^{\mathrm{f}, \mathrm{ft}, \geqslant 1}\right) \rightarrow \mathrm{dSt}_{A}^{\mathrm{f}}
$$

Because $\mathrm{C}_{A}$ preserves coproducts and suspensions, the functor $\mathcal{F}_{A}$ admits a right adjoint $\mathfrak{L}_{A}$ given by right-composing by $\mathrm{C}_{A}$. The composite functor

$$
\mathrm{dSt}_{A}^{\mathrm{f}} \stackrel{\mathfrak{L}_{A}}{\longrightarrow} \mathcal{P}_{\Sigma}^{\mathrm{st}}\left(\mathbf{d g L i e}_{A}^{\mathrm{f}, \mathrm{ft}, \geqslant 1}\right) \longrightarrow \mathcal{P}_{\Sigma}^{\mathrm{st}}\left(\operatorname{dgMod}_{A}^{\mathrm{f}, \mathrm{ft}, \geqslant 1}\right) \simeq \operatorname{dgMod}_{A}
$$

then corresponds to the functor

$$
X \mapsto X\left(\mathrm{C}_{A}(\operatorname{Free}(-))\right) \simeq X\left(A \oplus(-)^{\vee}[-1]\right)
$$

The use of remark 1.2.4 proves the first item. The functor

$$
\mathrm{C}_{A}:\left(\operatorname{dgLie}_{A}^{\mathrm{f}, \mathrm{ft}, \geqslant 1}\right)^{\mathrm{op}} \rightarrow \operatorname{dgExt}_{A}
$$


is essentially surjective. This implies that $\mathfrak{L}_{A}$ is conservative. We now focus on the third item. Because $\mathfrak{L}_{A}$ is conservative, it suffices to prove that the unit id $\rightarrow \mathfrak{L}_{A} \mathcal{F}_{A}$ is an equivalence. Let us consider $L \in$ $\mathbf{d g L i e}_{A}$ and study the map $L \rightarrow \mathfrak{L}_{A} \mathcal{F}_{A} L$. There exists a sifted diagram $\left(L_{\alpha}\right)$ of objects in $\mathbf{d g L i e}{ }_{A}^{\mathrm{f}, \mathrm{ft}, \geqslant 1}$ such that $L \simeq \operatorname{colim} L_{\alpha}$ in $\mathcal{P}_{\Sigma}\left(\mathbf{d g L i e}_{A}^{\mathrm{f}, \mathrm{ft}, \geqslant 1}\right)$ (and thus also in $\left.\mathcal{P}_{\Sigma}^{\mathrm{st}}\left(\mathbf{d g L i e}_{A}^{\mathrm{f}, \mathrm{ft}, \geqslant 1}\right) \simeq \mathbf{d g L i e}_{A}\right)$. This colimit is preserved by both $\mathcal{F}_{A}$ and $\mathfrak{L}_{A}$ and we cna therefore restrict to the case $L \in \mathbf{d g L i e}_{A}^{\mathrm{f}, \mathrm{ft}, \geqslant 1}$. The morphism $L \rightarrow \mathfrak{L}_{A} \mathcal{F}_{A} L$ is then equivalent to the adjunction unit $L \rightarrow \mathrm{D}_{A} \mathrm{C}_{A} L$. We conclude with lemma 1.4.12 using the noetherian assumption.

Until the end of this section, we will focus on proving that the definition we give of a formal stack is equivalent to Lurie's definition of a formal moduli problem in [DAG-X], as soon as the base dg-algebra $A$ is noetherian.

Definition 1.5.8. An augmented $A$-dg-algebra $B \in \mathbf{c d g a}_{A}^{\leqslant 0} / A$ is called artinian if there is sequence

$$
B=B_{0} \rightarrow \ldots \rightarrow B_{n}=A
$$

and for $0 \leqslant i<n$ an integer $p_{i} \geqslant 1$ such that

$$
B_{i} \simeq B_{i+1} \underset{A\left[\varepsilon_{p_{i}}\right]}{\times} A
$$

where $A\left[\varepsilon_{p_{i}}\right]$ denote the trivial square zero extension $A \oplus A\left[p_{i}\right]$.

We denote by $\operatorname{dgArt}_{A}$ the full subcategory of cdga $_{A}^{\leq 0} / A$ spanned by the artinian dg-algebras.

Definition 1.5.9. A formal moduli problem over $A$ is a functor

$$
X: \operatorname{dgArt} A_{A} \rightarrow \text { sSets }
$$

satisfying the conditions:

(F1) For $n \geqslant 1$ and $B \in \mathbf{d g A r t}_{A} / A\left[\varepsilon_{n}\right]$ the following natural morphism is an equivalence:

$$
X\left(\begin{array}{c}
B \times A \\
A\left[\varepsilon_{n}\right]
\end{array}\right) \stackrel{\sim}{\rightarrow} X(B) \times X(A)
$$

(F2) The simplicial set $X(A)$ is contractible.

Let $\widetilde{\mathbf{d S t}_{A}^{\mathrm{f}}}$ denote the full subcategory of $\mathcal{P}\left(\mathbf{d g} \mathbf{A r t} \mathbf{t}_{A}^{\mathrm{op}}\right)$ spanned by the formal moduli problems. This is an accessible localization of the presentable $(\infty, 1)$-category $\mathcal{P}\left(\mathbf{d g} \mathbf{A} \mathbf{r t}{ }_{A}^{\mathrm{op}}\right)$ of simplicial presheaves over $\operatorname{dg}_{\operatorname{Art}}^{\mathrm{op}}$.

Proposition 1.5.10. Let $A \in \mathbf{c d g a}_{k}^{\leqslant 0}$ be noetherian. The left Kan extension of the inclusion functor $i: \mathbf{d g E x t}_{A} \rightarrow \mathbf{d g} \mathbf{A r t} \mathbf{A}_{A}$ induces an equivalence of $(\infty, 1)$-categories

$$
j: \mathbf{d S t}_{A}^{\mathrm{f}} \rightarrow \widetilde{\mathbf{d S t}_{A}^{\mathrm{f}}}
$$

Proof. We will actually prove that the composed functor

$$
f: \operatorname{dgLie}_{A} \rightarrow \mathrm{dSt}_{A}^{\mathrm{f}} \rightarrow \widetilde{\mathrm{dSt}_{A}^{\mathrm{f}}}
$$

is an equivalence. The functor $f$ admits a right adjoint $g=\mathfrak{L}_{A} i^{*}$.

Given $n \geqslant 1$ and a diagram $B \rightarrow A\left[\varepsilon_{n}\right] \leftarrow A$ in $\operatorname{dgArt}_{A}$, lemma 1.4 .12 implies that the natural morphism

$$
\mathrm{D}_{A}(B) \underset{\mathrm{D}_{A}\left(A\left[\varepsilon_{n}\right]\right)}{\amalg} \mathrm{D}_{A}(A) \stackrel{\sim}{\rightarrow} \mathrm{D}_{A}\left(\begin{array}{c}
B \times A \\
A\left[\varepsilon_{n}\right]
\end{array}\right)
$$


is an equivalence. For any $B \in \mathbf{d g A r t}_{A}$ the adjunction morphism $\mathrm{C}_{A} \mathrm{D}_{A} B \rightarrow B \in\left(\mathbf{c d g a}_{A}^{\leqslant 0} / A\right)^{\mathrm{op}}$ is then an equivalence. Note that it is actually a map of augmented cdga's $B \rightarrow \mathrm{C}_{A} \mathrm{D}_{A} B$. Given $L \in \operatorname{dgLie}_{A}$ the functor $\mathrm{D}^{*} L: \operatorname{dg} \mathbf{A r t}_{A} \rightarrow \operatorname{sSets}$ defined by $\mathrm{D}^{*}(B)=\operatorname{Map}\left(\mathrm{D}_{A}(B), L\right)$ is a formal moduli problem (we use here the above equivalence). The natural morphism id $\rightarrow \mathrm{D}^{*} g$ of $\infty$-functors from $\mathbf{d S t}_{A}^{\mathrm{f}}$ to itself is therefore an equivalence. The same goes for the morphism $g \mathrm{D}^{*} \rightarrow$ id of $\infty$-functors from $\mathbf{d g L i e}_{A}$ to itself. The functor $g$ is an equivalence, so is $f$ and hence so is $j$.

\section{Tangent Lie algebra}

We now focus on gluing the functors built in the previous section, proving the following statement.

Theorem 2.0.1. Let $X$ be a derived Artin stack locally of finite presentation. Then there is a Lie algebra $\ell_{X}$ over $X$ whose underlying module is equivalent to the shifted tangent complex $\mathbb{T}_{X}[-1]$ of $X$.

Moreover if $f: X \rightarrow Y$ is a morphism between algebraic stacks locally of finite presentation then there is a tangent Lie morphism $\ell_{X} \rightarrow f^{*} \ell_{Y}$. More precisely, there is a functor

$$
X / \mathbf{d S t}_{k}^{\mathrm{Art}, \text { lfp }} \rightarrow \ell_{X} / \mathbf{d g L i e}_{X}
$$

sending a map $f: X \rightarrow Y$ to a morphism $\ell_{X} \rightarrow f^{*} \ell_{Y}$. The underlying map of quasi-coherent sheaves is indeed the tangent map (shifted by -1 ).

\subsection{Formal stacks and Lie algebras over a derived Artin stack}

Let $A \rightarrow B$ be a morphism in $\mathbf{c d g a}_{k}^{\leqslant 0}$. There is an exact scalar extension functor $B \otimes_{A}-: \mathbf{d g E x t}_{A} \rightarrow$ $\mathbf{d g E x t}_{B}$. Composing by this functor induces a restriction functor $\left(B \otimes_{A}-\right)^{*}: \mathbf{d S t}_{B}^{\mathrm{f}} \rightarrow \mathbf{d S t}_{A}^{\mathrm{f}}$. Moreover, the composite functor $\mathbf{d g E x t}_{A}^{\mathrm{op}} \rightarrow \mathbf{d g E x t}_{B}^{\mathrm{op}} \rightarrow \mathbf{d S t}_{B}^{\mathrm{f}}$ admits a left Kan extension (see [HTT, 5.5.8.15]) $\left(B \otimes_{A}-\right)_{!}: \mathbf{d} \mathbf{S} \mathbf{t}_{A}^{\mathrm{f}} \rightarrow \mathbf{d} \mathbf{S} \mathbf{t}_{B}^{\mathrm{f}}$. We actually get an adjunction

$$
(B \underset{A}{\otimes}-)_{!}: \mathbf{d S t}_{A}^{\mathrm{f}} \rightleftarrows \mathbf{d S t}_{B}^{\mathrm{f}}:(B \underset{A}{\otimes}-)^{*}
$$

We will now prove the stronger result (recall that $\operatorname{Pr}_{\infty}^{\mathrm{L}, \mathbb{U}}$ denotes the category of presentable categories with left adjoints as morphisms between them):

Proposition 2.1.1. There is a natural functor $\mathbf{d S t}^{\mathrm{f}}: \mathbf{c d g a}_{k}^{\leqslant 0} \rightarrow \mathbf{P r}_{\infty}^{\mathrm{L}, \mathbb{U}}$ mapping A to $\mathbf{d S t}_{A}^{\mathrm{f}}$. There moreover exists a natural transformation

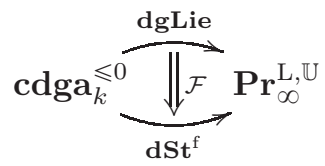

such that for any $A \in \mathbf{c d g a}_{k}^{\leqslant 0}$, the induced functor $\mathbf{d g L i e}_{A} \rightarrow \mathbf{d S t}_{A}^{\mathrm{f}}$ is equivalent to $\mathcal{F}_{A}$ as defined in proposition 1.5.6.

Proof. Let us recall the category $\int$ dgLie defined in the proof of proposition 1.2.6. Its objects are pairs $(A, L)$ where $A \in \operatorname{cdga}_{k}^{\leqslant 0}$ and $L \in \operatorname{dgLie}_{A}$.

We define $\int(\mathrm{cdga} /-)^{\mathrm{oP}}$ to be the following (1-)category.

- An object is a pair $(A, B)$ where $A \in \operatorname{cdga}_{k}^{\leqslant 0}$ and $B \in \operatorname{cdga}_{A} / A$.

- A morphism $(A, B) \rightarrow\left(A^{\prime}, B^{\prime}\right)$ is a map $A \rightarrow A^{\prime}$ together with a map $B^{\prime} \rightarrow B \otimes_{A}^{\mathbb{L}} A^{\prime}$ of $A^{\prime}$-dg-algebras. 
From definition 1.4.6, we get a functor $\mathrm{C}: \int \mathrm{dgLie} \rightarrow \int(\mathrm{cdga} /-)^{\mathrm{op}}$ preserving quasi-isomorphisms. This induces a diagram of $(\infty, 1)$-categories

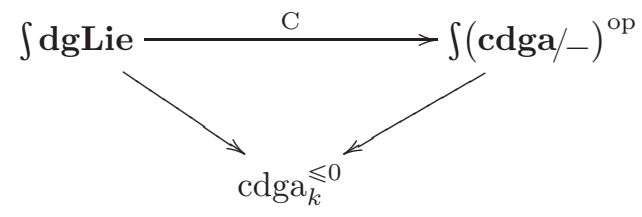

Restricting to the full subcategory spanned by pairs $(A, L)$ where $L \in \mathbf{d g L i e}_{A}^{\mathrm{f}, \mathrm{ft}, \geqslant 1}$, we get a functor

$$
\int d g L i e^{f, f t, \geqslant 1} \longrightarrow \text { C } \operatorname{dgExt}^{\mathrm{op}}
$$

Using lemma 1.4.10, we see that this last functor preserves coCartesian morphisms over cdga ${ }_{k}^{\leqslant 0}$. It defines a natural transformation between functors cdga ${ }_{k}^{\leqslant 0} \rightarrow \mathbf{C a t}_{\infty}^{\mathbb{U}}$. Since both the functors at hand map quasi-isomorphisms to categorical equivalences, it factors through the localisation cdga $_{k}^{\leqslant 0}$ of $\operatorname{cdga}_{k} \leqslant 0$. We now have a natural transformation

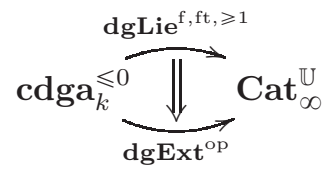

Composing with the functor $\mathcal{P}_{\Sigma}^{\text {st }}: \mathbf{C a t}_{\infty}^{\mathbb{U}} \rightarrow \mathbf{C a t}_{\infty}^{\mathbb{V}}$, we get a natural transformation $\mathcal{F}$ : dgLie $\simeq$ $\mathcal{P}_{\Sigma}^{\text {st }}\left(\mathbf{d g L i e}^{\mathrm{f}, \mathrm{ft}, \geqslant 1}\right) \rightarrow \mathbf{d S t}^{\mathrm{f}}$. Moreover, for any $A \in \mathbf{c d g a}_{k}^{\leqslant 0}$, both $\mathbf{d g L i e}_{A}$ and $\mathbf{d S t}_{A}^{\mathrm{f}}$ are presentable and the induced functor $\mathcal{F}_{A}: \mathbf{d g L i e}_{A} \rightarrow \mathbf{d S t}_{A}^{\mathrm{f}}$ admits a right adjoint (see proposition 1.5.6).

Remark 2.1.2. The Grothendieck construction defines a functor

$$
\mathcal{F}: \int \operatorname{dgLie} \longrightarrow \int d \mathrm{St}^{\mathrm{f}}
$$

over $\mathbf{c d g a}_{k}^{\leqslant 0}$. Note that we also have a composite functor

$$
G: \int \operatorname{dgLie} \stackrel{\mathrm{C}}{\longrightarrow} \int(\mathbf{c d g a} /-)^{\mathrm{op}} \stackrel{h}{\longrightarrow} \int \mathrm{dSt}^{\mathrm{f}}
$$

where $h$ is deduced from the Yoneda functor. The functor $\mathcal{F}$ is by definition the relative left Kan extension of the restriction of $G$ to $\int \mathbf{d g L i e}{ }^{\mathrm{f}, \mathrm{ft}, \geqslant 1}$. It follows that we have a natural transformation $\mathcal{F} \rightarrow G$. We will use that fact a few pages below.

Proposition 2.1.3. The functor

$$
\text { dgLie }: \operatorname{cdga}_{k}^{\leqslant 0} \rightarrow \operatorname{Pr}_{\infty}^{\mathrm{L}, \mathbb{U}}
$$

is a stack for the fpqc topology.

Proof. The functor dgLie is endowed with a forgetful natural transformation to dgMod, the stack of dg-modules. This forgetful transformation is pointwise conservative and preserves limits. This implies that dgLie is also a stack.

Definition 2.1.4. Let $X$ be an algebraic derived stack. The $(\infty, 1)$-category $\mathbf{d S t}_{X}^{\mathrm{f}}$ of formal stacks over $X$ is the limit of the diagram

$$
\left(\mathbf{d A f f}_{k} / X\right)^{\mathrm{op}} \longrightarrow \mathbf{d A f f}_{k}^{\mathrm{op}}=\operatorname{cdga}_{k}^{\leqslant 0} \stackrel{\mathrm{dSt}^{\mathrm{f}}}{\longrightarrow} \operatorname{Pr}_{\infty}^{\mathrm{L}, \mathbb{U}}
$$


Similarly, the $(\infty, 1)$-category $\mathbf{d g L i e}_{X}$ of dg-Lie algebras over $X$ is the limit of

$$
\left(\mathbf{d A f f}_{k} / X\right)^{\mathrm{op}} \longrightarrow \mathbf{d A f f}_{k}^{\mathrm{op}}=\mathbf{c d g a}_{k}^{\leqslant 0} \stackrel{\text { dgLie }}{\longrightarrow} \mathbf{P r}_{\infty}^{\mathrm{L}, \mathbb{U}}
$$

The natural transformation $\mathcal{F}: \mathbf{d g L i e} \rightarrow \mathbf{d S t}^{\mathrm{f}}$ induces a functor

$$
\mathcal{F}_{X}: \operatorname{dgLie}_{X} \rightarrow \mathrm{dSt}_{X}^{\mathrm{f}}
$$

By definition, it admits a right adjoint which we will denote by $\mathfrak{L}_{X}$.

Remark 2.1.5. Of course, for any $A \in \mathbf{c d g a} a_{k}^{\leqslant 0}$ we get $\mathbf{d S t}_{\text {Spec } A}^{\mathrm{f}} \simeq \mathbf{d S t}_{A}^{\mathrm{f}}$ and $\mathbf{d g L i e}_{\text {Spec } A} \simeq \mathbf{d g L i e}_{A}$ since the above diagrams have an initial object.

Remark 2.1.6. The functor $\mathfrak{L}_{X}$ may not commute with base change.

\subsection{Tangent Lie algebra of a derived Artin stack locally of finite presenta- tion}

We start be using the construction we described in remark 1.2.8. Let us consider $\mathcal{C}$ the following category.

- An object is a pair $(A, F \rightarrow G)$ where $A$ is in $\operatorname{cdga}_{k}^{\leqslant 0}$ and $F \rightarrow G$ is a morphism in the model category of simplicial presheaves over $\left(\operatorname{cdga}_{A}^{\leqslant 0}\right)^{\mathrm{op}}$.

- A morphism $(A, F \rightarrow G) \rightarrow\left(B, F^{\prime} \rightarrow G^{\prime}\right)$ is the datum of a morphism $A \rightarrow B$ together with a commutative square

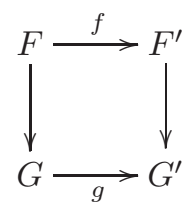

of presheaves over $\left(\operatorname{cdga}_{B}^{\leq 0}\right)^{\text {op }}$

A map in $\mathcal{C}$ as above is a weak equivalence if the morphism $A \rightarrow B$ is an identity and if the maps $f$ and $g$ are weak equivalences in the model category of simplicial presheaves. We set $\int \mathcal{P}(\mathbf{d A f f})^{\Delta^{1}}$ to be the $(\infty, 1)$-localization of $\mathcal{C}$ along weak equivalences. The natural functor $\int \mathcal{P}(\mathbf{d} \mathbf{A} f f)^{\Delta^{1}} \rightarrow \operatorname{cdga}_{k}^{\leqslant 0}$ is a coCartesian fibration classified by the functor $A \mapsto \mathcal{P}\left(\mathbf{d} \mathbf{A} f f{ }_{A}\right)^{\Delta^{1}}$.

Let $\mathcal{D}$ denote the following category.

- An object is a pair $(A, F)$ where $F$ is a simplicial presheaf over the opposite category of morphisms in $\operatorname{cdga}{ }_{A}^{\leq 0}$.

- A morphism $(A, F) \rightarrow(B, G)$ is a morphism $A \rightarrow B$ and a map $F \rightarrow G$ as simplicial presheaves over $\left(\operatorname{cdga}_{B}^{\leqslant 0}\right)^{\mathrm{op}} \Delta^{1}$.

A map in $\mathcal{D}$ as above is a weak equivalence if the morphism $A \rightarrow B$ is an identity and if $F \rightarrow G$ is a weak equivalence in the model category of simplicial presheaves. We will denote by $\int \mathcal{P}\left(\mathbf{d A f f}^{\Delta^{1}}\right)$ the $(\infty, 1)$-category obtained from $\mathcal{D}$ by localizing along weak equivalences. The natural functor $\int \mathcal{P}\left(\mathbf{d A f f}^{\Delta^{1}}\right) \rightarrow \operatorname{cdga}_{k}^{\leqslant 0}$ is a coCartesian fibration.

Lemma 2.2.1. There is a relative adjunction

$$
f: \int \mathcal{P}\left(\mathbf{d A f f}^{\Delta^{1}}\right) \rightleftarrows \int \mathcal{P}(\mathbf{d} \mathbf{A f f})^{\Delta^{1}}: g
$$


over $\operatorname{cdga}_{k}^{\leqslant 0}$. They can be described on the fibers as follows. Let $A \in \operatorname{cdga}_{k}^{\leqslant 0}$. The left adjoint $f_{A}$ is given on morphisms between affine schemes to the corresponding morphism of representable functors. The right adjoint $g_{A}$ maps a morphism $F \rightarrow G$ to the representable simplicial presheaf

$$
\operatorname{Map}(-, F \rightarrow G)
$$

Proof. Let us define a functor $\mathcal{C} \rightarrow \mathcal{D}$ mapping $(A, F \rightarrow G)$ to the functor

$$
(S \rightarrow T) \mapsto \operatorname{Map}(S \rightarrow T, F \rightarrow G)
$$

We can now derive this functor (replacing therefore $F \rightarrow G$ with a fibrant replacement). We get a functor

$$
g: \int \mathcal{P}(\mathbf{d A f f})^{\Delta^{1}} \rightarrow \int \mathcal{P}\left(\mathbf{d A f f}^{\Delta^{1}}\right)
$$

which commutes with the projections to $\operatorname{cdga}_{k}^{\leqslant 0}$. Let $A$ be in $\operatorname{cdga}_{k}^{\leqslant 0}$ and let $g_{A}$ be the induced functor

$$
\mathcal{P}\left(\mathbf{d A f f}_{A}\right)^{\Delta^{1}} \rightarrow \mathcal{P}\left(\mathbf{d A f f}_{A}^{\Delta^{1}}\right)
$$

It naturally admits a left adjoint. Namely the left Kan extension $f_{A}$ to the Yoneda embedding

$$
\mathbf{d A f f}_{A}^{\Delta^{1}} \rightarrow \mathcal{P}\left(\mathbf{d A f f}_{A}\right)^{\Delta^{1}}
$$

For any morphism $A \rightarrow B$ in $\operatorname{cdga}_{k}^{\leqslant 0}$, there is a canonical morphism

$$
f_{B}((B \underset{A}{\otimes}-), X) \rightarrow(B \underset{A}{\otimes}-)_{!} f_{A}(X)
$$

which is an equivalence. [When $X=\operatorname{Spec} A^{\prime} \rightarrow \operatorname{Spec} A^{\prime \prime}$ is representable then both left and right hand sides are equivalent to $\operatorname{Spec} B^{\prime} \rightarrow \operatorname{Spec} B^{\prime \prime}$ where $B^{\prime}=B \otimes_{A} A^{\prime}$ and $\left.B^{\prime \prime}=B \otimes_{A} A^{\prime \prime}\right]$. We complete the proof using [HAlg, 8.2.3.11].

Let now $\int * / \mathcal{P}(\mathbf{d A f f}) \rightarrow \mathbf{c d g a}_{k}^{\leqslant 0}$ denote the coCartesian fibration classified by the subfunctor $A \mapsto \operatorname{Spec}(A) / \mathcal{P}\left(\mathbf{d} \mathbf{A f f}{ }_{A}\right)$ of $\mathcal{P}(\mathbf{d} \mathbf{A f f})^{\Delta^{1}}$. Let also $\int \mathcal{P}\left(\mathbf{d} \mathbf{A f f}{ }^{*}\right)$ be defined similarly to $\int \mathcal{P}\left(\mathbf{d} \mathbf{A f f}{ }^{\Delta^{1}}\right)$. It is classified by a functor

$$
A \mapsto \mathcal{P}\left(\operatorname{Spec} A / \mathrm{dAff}_{A}\right)
$$

Proposition 2.2.2. The adjunction of lemma 2.2.1 induces a relative adjunction

$$
\int \mathcal{P}\left(\text { dAff }^{*}\right) \rightleftarrows \int * / \mathcal{P}(\text { dAff })
$$

over $\operatorname{cdga}_{k}^{\leqslant 0}$. It moreover induces a natural transformation

$$
\operatorname{coga}_{k} \underset{* / \mathcal{P}(\mathrm{dAff})}{\stackrel{\mathcal{P}\left(\mathrm{dAff}^{*}\right)}{\| 0}} \mathrm{Cat}_{\infty}^{\mathbb{V}}
$$

Proof. We define the restriction functor

$$
\int \mathcal{P}\left(\mathbf{d A f f}^{\Delta^{1}}\right) \rightarrow \int \mathcal{P}\left(\mathbf{d A f f}^{*}\right)
$$

It admits a fiberwise left adjoint, namely the left Kan extension, which commutes with base change. This defines - using [HAlg, 8.3.2.11] - a relative left adjoint

$$
\int \mathcal{P}\left(\mathbf{d A f f}^{*}\right) \rightarrow \int \mathcal{P}\left(\mathbf{d A f f}^{\Delta^{1}}\right)
$$


Composing with the relative adjunction of lemma 2.2.1, we get a relative adjunction

$$
\int \mathcal{P}\left(\mathbf{d A f f}^{*}\right) \rightleftarrows \int \mathcal{P}(\mathbf{d A f f})^{\Delta^{1}}
$$

The left adjoint factors through $\int * / \mathcal{P}(\mathbf{d A f f})$ and the composed functor

$$
\int * / \mathcal{P}(\text { dAff }) \rightarrow \int \mathcal{P}(\text { dAff })^{\Delta^{1}} \rightarrow \int \mathcal{P}\left(\text { dAff }^{*}\right)
$$

is its relative right adjoint. It follows that the functor $\int \mathcal{P}\left(\mathbf{d A f f}^{*}\right) \rightarrow \int * / \mathcal{P}(\mathbf{d A f f})$ preserves coCartesian morphisms over $\operatorname{cdga}_{k}^{\leqslant 0}$. We get a natural transformation

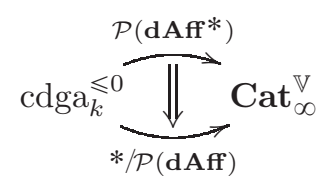

As both functors at hand map quasi-isomorphisms of cdga's to equivalences of categories, it factors through the localisation $\mathbf{c d g a}_{k}^{\leqslant 0}$ of $\operatorname{cdga}_{k}^{\leqslant 0}$.

Proposition 2.2.3. Let $X$ be an algebraic derived stack. There are functors

$$
\phi: X / \mathcal{P}\left(\mathbf{d A f f}_{k}\right) / X \rightarrow \lim _{\operatorname{Spec} A \rightarrow X} \operatorname{Spec} A / \mathcal{P}\left(\mathbf{d A f f}{ }_{A}\right)
$$

and

$$
\theta: \lim _{\operatorname{Spec} A \rightarrow X} \operatorname{Spec} A / \mathcal{P}\left(\mathbf{d} \mathbf{A f f}_{A}\right) \rightarrow \lim _{\operatorname{Spec} A \rightarrow X} \mathcal{P}\left(\mathbf{d A f f}_{A}^{*}\right)
$$

Proof. The functor $\phi$ is given by the following construction:

$$
\left.X / \mathcal{P}(\mathbf{d} \mathbf{A f f})_{k}\right) / X \lim _{\operatorname{Spec} A \rightarrow X} \operatorname{Spec} A / \mathcal{P}\left(\mathbf{d} \mathbf{A f f}_{k}\right) / \operatorname{Spec} A \simeq \lim _{\operatorname{Spec} A \rightarrow X} \operatorname{Spec} A / \mathcal{P}\left(\mathbf{d} \mathbf{A f f}{ }_{A}\right)
$$

The second functor is constructed as follows. From proposition 2.2.2 we get a functor

$$
\lim _{\operatorname{Spec} A \rightarrow X} \mathcal{P}\left(\mathbf{d} \mathbf{A f f} f_{A}^{*}\right) \rightarrow \lim _{\operatorname{Spec} A \rightarrow X} \operatorname{Spec} A / \mathcal{P}\left(\mathbf{d A f f}_{A}\right)
$$

It preserves colimits and both left and right hand sides are presentable. It thus admits a right adjoint $\theta$.

Remark 2.2.4. The functor $\theta$ is the limit of the functors

$$
(\operatorname{Spec} A \rightarrow F) \mapsto \operatorname{MapSpec}_{A} / \mathcal{P}\left(\mathbf{d A f f}_{A}\right)(-, \operatorname{Spec} A \rightarrow F)
$$

This construction commutes with base change. We can indeed draw the commutative diagram (where $S \rightarrow T$ is a morphism between affine derived schemes)

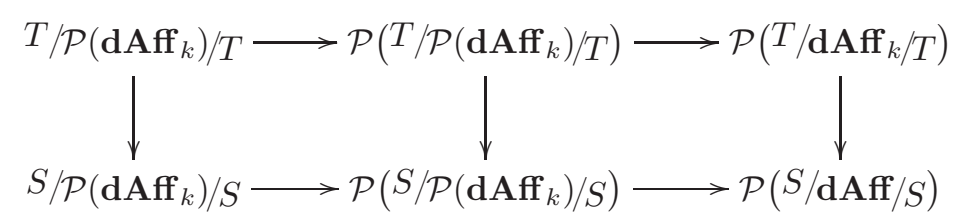

The left hand side square commutes by definition of base change. The right hand side square also commutes as the restriction along a fully faithful functor preserves base change. 
Definition 2.2.5. Let $X$ be a derived stack. Let us define the formal completion functor

$$
(-)^{\mathrm{f}}: X / \mathcal{P}\left(\mathbf{d A f f}{ }_{k}\right) / X \rightarrow \lim _{\operatorname{Spec} A \rightarrow X} \mathcal{P}\left(\left(\mathbf{d g E x} \mathbf{E t}_{A}\right)^{\mathrm{op}}\right)
$$

as the composed functor

$$
\begin{aligned}
X / \mathcal{P}\left(\mathbf{d A f f}_{k}\right) / X & \rightarrow \lim _{\operatorname{Spec} A \rightarrow X} \operatorname{Spec} A / \mathcal{P}\left(\mathbf{d A f f}_{A}\right) \\
& \rightarrow \lim _{\operatorname{Spec} A \rightarrow X} \mathcal{P}\left(\mathbf{d A f f}_{A}^{*}\right) \\
& \rightarrow \lim _{\operatorname{Spec} A \rightarrow X} \mathcal{P}\left(\left(\mathbf{d g E x t}_{A}\right)^{\mathrm{op}}\right)
\end{aligned}
$$

Remark 2.2.6. Let $u: S=\operatorname{Spec} A \rightarrow X$ be a point. The functor $u^{*}(-)^{\mathrm{f}}$ maps a pointed stack $Y$ over $X$ to the functor $\operatorname{dgExt}_{A} \rightarrow \mathbf{s S e t s}$

$$
B \mapsto \operatorname{Map}_{S /-/ X}(\operatorname{Spec} B, Y)
$$

Definition 2.2.7. Let $X$ be a derived stack. Let $\mathbf{d S t}_{X}^{*, \text { Art }}$ denote the full sub-category of $X / \mathcal{P}(\mathbf{d A f f}) / X$ spanned by those $X \rightarrow Y \rightarrow X$ such that $Y$ is a derived Artin stack over $X$.

Lemma 2.2.8. The restriction of $(-)^{\mathrm{f}}$ to $\mathbf{d S t}_{X}^{*, \text { Art }}$ has image in $\mathbf{d S t}_{X}^{\mathrm{f}}$.

Proof. We have to prove that whenever $X \stackrel{f}{\rightarrow} Y \rightarrow X$ is a pointed algebraic stack over $X$ then $Y^{f}$ is formal over $X$. Because of remark 2.2.4, it suffices to treat the case of an affine base. Let us assume $X=\operatorname{Spec} A$. The result follows from the existence of a relative cotangent complex $\mathbb{L}_{Y / X}$ :

$$
\begin{aligned}
Y^{\mathrm{f}}((A \oplus M) \underset{A}{\times}(A \oplus N)) & \simeq Y^{\mathrm{f}}(A \oplus(M \oplus N)) \simeq \operatorname{Map}_{X /-/ X}(\operatorname{Spec}(A \oplus(M \oplus N)), Y) \\
& \simeq \operatorname{Map}\left(f^{*} \mathbb{L}_{Y / X}, M \oplus N\right) \simeq \operatorname{Map}\left(f^{*} \mathbb{L}_{Y / X}, M\right) \times \operatorname{Map}\left(f^{*} \mathbb{L}_{Y / X}, N\right) \\
& \simeq Y^{\mathrm{f}}(A \oplus M) \times Y^{\mathrm{f}}(A \oplus M) \\
Y^{\mathrm{f}}(\underset{A \oplus M[1]}{A \underset{A}{\times} A)} & \simeq Y^{\mathrm{f}}(A \oplus M) \simeq \operatorname{Map}\left(f^{*} \mathbb{L}_{Y / X}, M\right) \\
& \simeq \Omega \operatorname{Map}\left(f^{*} \mathbb{L}_{Y / X}, M[1]\right) \simeq \Omega Y^{\mathrm{f}}(A \oplus M[1])
\end{aligned}
$$

Remark 2.2.9. Let $X=\operatorname{Spec} A$ and let $X \stackrel{y}{\rightarrow} Y \rightarrow X \in \mathbf{d S t}_{X}^{* \text {,Art }}$. Let us assume that $Y$ is locally of finite presentation over $A$. The tangent $\mathbb{T}_{Y^{\mathrm{f}} / X, y}$ of the formal stack $Y^{\mathrm{f}}$ over $X$ (see definition 1.5.4) is equivalent to the tangent $\mathbb{T}_{Y / X, y}$ of $Y$ at $y$ over $X$. By definition (see [HAG2, 1.4.1.14]), the tangent complex $\mathbb{T}_{Y / X, y}$ corepresents the functor

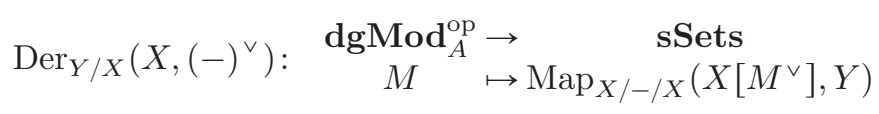

where $X\left[M^{\vee}\right]$ is the trivial square zero extension $\operatorname{Spec}\left(A \oplus M^{\vee}\right)$. Using proposition 1.2.2, we know it is actually determined by the restriction of $\operatorname{Der}_{Y / X}\left(X,(-)^{\vee}\right)$ to $\left(\operatorname{dgMod}_{A}^{\mathrm{f}, \mathrm{ft}, \geqslant 1}\right)^{\text {op }}$. On the other hand, the formal stack $Y^{\mathrm{f}}$ is the functor

$$
\begin{array}{cl}
\operatorname{dgExt}_{A} & \rightarrow \quad \text { SSets } \\
B & \mapsto \operatorname{Map}_{X /-/ X}(\operatorname{Spec} B, Y)
\end{array}
$$

Our claim follows from definition 1.5.4. 
Definition 2.2.10. Let $X$ be an algebraic stack locally of finite presentation. We define its tangent Lie algebra as the $X$-dg-Lie algebra

$$
\ell_{X}=\mathfrak{L}_{X}\left((X \times X)^{\mathrm{f}}\right)
$$

where the product $X \times X$ is a pointed stack over $X$ through the diagonal $\Delta: X \rightarrow X \times X$ and the first projection.

Proof (of theorem 2.0.1). Since $X$ has a perfect tangent complex, for any $u: S=\operatorname{Spec} A \rightarrow X$, the canonical map $\Gamma_{u}^{*} \mathbb{T}_{S \times X / S} \rightarrow u^{*} \Delta^{*} \mathbb{T}_{X \times X / X}$ is an equivalence (here $\Gamma_{u}: S \rightarrow S \times X$ denotes the graph of $u)$. We study the underlying $X$-dg-module of $\mathfrak{L}_{X}\left((X \times X)^{\mathrm{f}}\right)$. It represents the functor $\operatorname{dgMod}_{X}^{\mathrm{op}} \rightarrow$ sSets

$$
\begin{aligned}
M \mapsto \operatorname{Map}\left(\mathcal{F}_{X}\left(\operatorname{Free}_{X} M\right),(X \times X)^{\mathrm{f}}\right) & \simeq \lim _{u: \operatorname{Spec} A \rightarrow X} \operatorname{Map}\left(\mathcal{F}_{A}\left(\operatorname{Free}_{A}\left(u^{*} M\right)\right),(\operatorname{Spec} A \times X)^{\mathrm{f}}\right) \\
& \simeq \lim _{u: \operatorname{Spec} A \rightarrow X} \operatorname{Map}\left(u^{*} M, \operatorname{Forget}_{A} \mathfrak{L}_{A}\left((\operatorname{Spec} A \times X)^{\mathrm{f}}\right)\right) \\
& \simeq \lim _{u: \operatorname{Spec} A \rightarrow X} \operatorname{Map}\left(u^{*} M, \Gamma_{u}^{*} \mathbb{T}_{\operatorname{Spec} A \times X / \operatorname{Spec} A}\right) \\
& \simeq \lim _{u: \operatorname{Spec} A \rightarrow X} \operatorname{Map}\left(u^{*} M, u^{*} \Delta^{*} \mathbb{T}_{X \times X / X}[-1]\right) \\
& \simeq \operatorname{Map}\left(M, \Delta^{*} \mathbb{T}_{X \times X / X}[-1]\right)
\end{aligned}
$$

Let us precise that the equivalence between the second and the third line is obtained using proposition 1.5.6 and remark 2.2.9. We conclude that the underlying $X$-dg-module of $\mathfrak{L}_{X}\left((X \times X)^{\mathrm{f}}\right)$ is indeed $\Delta^{*} \mathbb{T}_{X \times X / X}[-1] \simeq$ $\mathbb{T}_{X}[-1]$.

Let us now consider the functor

$$
X / \mathbf{d S t}^{\mathrm{Art}, l \mathrm{lfp}} \rightarrow \mathrm{dSt}_{X}^{*, \mathrm{Art}}
$$

mapping a morphism $X \rightarrow Z$ to the stack $X \times Z$ pointed by the graph map $X \rightarrow X \times Z$ and endowed with the projection morphism to $X$. Composing this functor with $(-)^{\mathrm{f}}$ and $\mathfrak{L}_{X}$ we finally get the wanted functor

$$
X / \mathbf{d S t}^{\mathrm{Art}, \mathrm{lfp}} \rightarrow{ }^{\ell_{X} / \mathbf{d g L i e}_{X}}
$$

Let $f: X \rightarrow Z$ be in $X / \mathbf{d S t}^{\text {Art,lfp }}$. Since $Z$ is locally of finite presentation, its tangent is perfect and the canonical map

$$
\beta: \Gamma_{f}^{*} \mathbb{T}_{X \times Z / X} \rightarrow f^{*} \Delta_{Z}^{*} \mathbb{T}_{Z \times Z / Z}
$$

is an equivalence. At the level of Lie algebras, we have a canonical map

$$
\alpha: u^{*} \ell_{Z} \rightarrow \mathfrak{L}_{X}\left((X \times Z)^{\mathrm{f}}\right)
$$

The map of $X$-dg-modules underlying $\alpha$ is equivalent to $\beta[-1]$. Since the forgetful functor $\mathbf{d g L i e}{ }_{X} \rightarrow$ $\operatorname{dgMod}_{X}$ is conservative, we deduce that $\alpha$ is an equivalence.

Remark 2.2.11. We also proved above that for any map $u: X \rightarrow Z$ between locally finitely presented derived Artin stacks, the canonical map

$$
u^{*} \ell_{Z} \rightarrow \mathfrak{L}_{X}\left((X \times Z)^{\mathrm{f}}\right)
$$

is an equivalence. 


\subsection{Derived categories of formal stacks}

The goal of this subsection is to prove the following

Theorem 2.3.1. Let $X$ be an algebraic stack locally of finite presentation. There is a colimit preserving monoidal functor

$$
\operatorname{Rep}_{X}: \operatorname{Qcoh}(X) \rightarrow \operatorname{dgRep}_{X}\left(\ell_{X}\right)
$$

where $\operatorname{dgRep}_{X}\left(\ell_{X}\right)$ is the $(\infty, 1)$-category of representations of $\ell_{X}$. Moreover, the functor $\operatorname{Rep}_{X}$ is a retract of the forgetful functor.

We will prove this theorem at the end of the subsection. For now, let us state and prove a few intermediate results. Let $A$ be any $\operatorname{cdga}_{k}^{\leqslant 0}$ and $L \in \operatorname{dgLie}_{A}$. The category $\operatorname{dgRep}_{A}(L)$ of representations of $L$ is endowed with a combinatorial model structure for which equivalences are exactly the $L$-equivariant quasi-isomorphisms and for which the fibrations are those maps sent onto fibrations by the forgetful functor to $\operatorname{dgMod}_{A}$.

Definition 2.3.2. Let us denote by $\operatorname{dgRep}_{A}(L)$ the underlying $(\infty, 1)$-category of the model category $\operatorname{dgRep}_{A}(L)$.

Lemma 2.3.3. Let $L$ be an A-dg-Lie algebra. There is a Quillen adjunction

$$
f_{L}^{A}: \operatorname{dgMod}_{\mathrm{C}_{A} L} \rightleftarrows \operatorname{dgRep}_{A}(L): g_{L}^{A}
$$

Given by

$$
\begin{aligned}
& f_{L}^{A}: V \mapsto \mathcal{U}_{A}(A[\eta] \underset{A}{\otimes} L) \underset{\mathrm{C}_{A} L}{\otimes} V \\
& g_{L}^{A}: M \mapsto \underline{\operatorname{Hom}}_{L}\left(\mathcal{U}_{A}(A[\eta] \underset{A}{\otimes} L), M\right)
\end{aligned}
$$

where $A[\eta] \otimes_{A} L$ is as in subsection 1.4 and $\underline{\operatorname{Hom}}_{L}$ denotes the mapping complex of dg-representations of $L$.

Remark 2.3.4. The image $g_{L}^{A}(M)$ is a model for the cohomology $\mathbb{R} \underline{\operatorname{Hom}}_{L}(A, M)$ of $L$ with values in $M$. We can give an explicit description of $g_{L}^{A}(M)$ as a graded module, similarly to remark 1.4.2:

$$
g_{L}^{A}(M) \simeq \underline{\operatorname{Hom}}_{A}(\operatorname{Sym}(L[1]), M)
$$

The differentials differ though. As the one on $g_{L}^{A}(M)$ encodes part of the action of $L$ on $M$.

Proof. The fact that $f_{L}^{A}$ and $g_{L}^{A}$ are adjoint functors is immediate. The functor $f_{L}^{A}$ preserves quasiisomorphisms (see the proof of proposition 1.4.3) and fibrations. This is therefore a Quillen adjunction.

Remark 2.3.5. The category $\operatorname{dgRep}_{A}(L)$ is endowed with a symmetric tensor product. If $M$ and $N$ are two dg-representations of $L$, then $M \otimes_{A} N$ is endowed with the diagonal action of $L$. The category $\operatorname{dgMod}_{\mathrm{C}_{A} L}$ is also symmetric monoidal. Moreover, for any pair of $L$-dg-representations $M$ and $N$, there is a natural morphism

$$
g_{L}^{A}(M) \underset{\mathrm{C}_{A} L}{\otimes} g_{L}(N) \rightarrow g_{L}^{A}(M \underset{A}{\otimes} N)
$$

This makes $g_{L}^{A}$ into a weak monoidal functor. In particular, the functor $g_{L}^{A}$ defines a functor $\operatorname{dgLie}_{L} \rightarrow$ $\operatorname{dgLie}_{\mathrm{C}_{A}(L)}$, also denoted $g_{L}^{A}$.

Proposition 2.3.6. Let $L$ be a good dg-Lie algebra over $A$. The induced functor

$$
f_{L}^{A}: \operatorname{dgMod}_{\mathrm{C}_{A} L} \rightarrow \operatorname{dgRep}_{A}(L)
$$

of $(\infty, 1)$-categories is fully faithful. 
Remark 2.3.7. The above proposition can be seen as a consequence of some general Morita theory statement. The adjunction at hand is induced by the $\mathcal{U}_{A} L \otimes \mathrm{C}_{A} L$-bimodule $A$ where $\mathrm{C}_{A} L=$ $\underline{\operatorname{Hom}}_{\mathcal{U}_{A} L}(A, A)$. In such a case, the left adjoint $f_{L}^{A}$ is fully faithful is and only if $A$ is a perfect $\mathcal{U}_{A} L$ dg-module. The assumption that $L$ is good ensures the perfection of $A$ as a left $\mathcal{U}_{A} L$-dg-module.

More generally, let $C$ be an associative $A$-dg-algebra. Let us assume $C$ is a finite cellular object in the category of $A$-dg-algebras: there is a finite sequence

$$
A \simeq C_{0} \rightarrow C_{1} \rightarrow \ldots \rightarrow C_{n} \simeq C
$$

such that for any $1 \leqslant i \leqslant n$, the map $C_{i-1} \rightarrow C_{i}$ fits into a coCartesian square

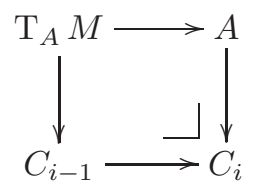

where $T_{A} M$ is the tensor algebra generated by a free $A$-dg-module $M$ of finite type. If moreover $C$ is equipped with an augmentation $C \rightarrow A$ then the left $C$-dg-module $A$ is perfect. To prove this, let us assume $A$ is perfect as a left $C_{i-1}$-dg-module: $A$ is a finite colimit of free $C_{i-1}$-dg-modules. It follows that $\mathrm{T}_{A}(M[1]) \simeq C_{i} \otimes_{C_{i-1}} A$ is a finite colimit of free $C_{i}$-dg-modules. We then obtain $A$ by moding out the generators of $\mathrm{T}_{A}(M[1])$, again a finite colimit.

Proof (of proposition 2.3.6). This proof is very similar to that of [DAG-X, 2.4.12]. In this proof, we will write $f$ instead of $f_{L}^{A}$. Let $B$ denote $C_{A} L$. We first prove that the restriction $f_{\mid \operatorname{Perf}(B)}$ is fully faithful. Let $V$ and $W$ be two $B$-dg-modules. There is a map $\operatorname{Map}(V, W) \rightarrow \operatorname{Map}(f V, f W)$. Fixing $V$ (resp. $W$ ), the set of $W$ 's (resp. $V$ 's) such that this map is an equivalence is stable under extensions, shifts and retracts. It is therefore sufficient to prove that the map $\operatorname{Map}(B, B) \rightarrow \operatorname{Map}(f B, f B)$ is an equivalence, which follows from the definition (if we look at the dg-modules of morphisms, then both domain and codomain are quasi-isomorphic to $\left.B=\mathrm{C}_{A} L\right)$.

To prove that $f: \mathbf{d g M o d}_{B} \rightarrow \operatorname{dg} \operatorname{Rep}_{A}(L)$ is fully faithful, we only need to prove that $f$ preserves compact objects. It suffices to prove that $f B \simeq A$ is compact in $\operatorname{dgRep}_{A}(L)$. The (non commutative) $A$-dg-algebra $\mathcal{U}_{A}(L)$ is a finite cellular object (because $L$ is good) and is endowed with an augmentation morphism to $A$. It follows that $A$, seen as a left $\mathcal{U}_{A}(L)$-dg-module through the augmentation, is a finite cellular object (see remark 2.3.7) and hence is compact. The forgetful functor $\operatorname{dgMod}_{A} \rightarrow$ $\operatorname{dgMod}_{\mathcal{U}_{A}(L)}^{\text {left }}$ therefore preserves compact objects.

Let us now study the behaviour of the adjunction $\left(f_{L}^{A}, g_{L}^{A}\right)$ with respect to change of base $A$ and of Lie algebra $L$. We will as the same time consider the compatibility with the monoidal structures. Once more, we will use the procedure of remark 1.2.8. Let us consider the category $\int d g L i e^{o p}$ such $^{\text {op }}$ that:

- An object is a pair $(A, L)$ with $A \in \operatorname{cdga}_{k}^{\leqslant 0}$ and with $L \in \operatorname{dgLie}_{A}$ and

- A morphism $(A, L) \rightarrow\left(B, L^{\prime}\right)$ is a map $A \rightarrow B$ together with a map $L^{\prime} \rightarrow L \otimes_{A}^{\mathbb{L}} B$ in $\operatorname{dgLie}_{B}$.

It is endowed with a functor $\int d g L i e^{\text {op }} \rightarrow \operatorname{cdga}_{k}^{\leqslant 0}$. We will say that a map in $\int d g L i e^{\text {op }}$ is a weak equivalence if the map of cdga's is an identity and the map of dg-Lie algebras is a quasi-ismorphism. Localising along weak equivalences, we obtain a coCartesian fibration of $(\infty, 1)$-categories

$$
\int \operatorname{dgLie}^{\mathrm{op}} \rightarrow \operatorname{cdga}_{k}^{\leqslant 0}
$$

classified by the functor $A \mapsto \mathbf{d g L i e}{ }_{A}^{\text {op }}$ (see the proof of proposition 1.2.6).

Let Fin* denote the category of pointed finite sets - see definition 0.0.1. For $n \in \mathbb{N}$, we will denote by $\langle n\rangle$ the finite set $\{*, 1, \ldots, n\}$ pointed at $*$. Let $\int \operatorname{dgRep}{ }^{\otimes}$ be the following category. 
- An object is a family $\left(A, L, M_{1}, \ldots, M_{m}\right)$ with $A \in \operatorname{cdga}_{k}^{\leqslant 0}$, with $L \in \operatorname{dgLie}_{A}$ and with $M_{i} \in$ $\operatorname{dgRep}_{A}(L)$.

- A morphism $\left(A, L, M_{1}, \ldots, M_{m}\right) \rightarrow\left(B, L^{\prime}, N_{1}, \ldots, N_{n}\right)$ is the datum of a map $(A, L) \rightarrow\left(B, L^{\prime}\right) \in$ $\int \mathrm{dgLie}^{\mathrm{op}}$, of a map $t:\langle m\rangle \rightarrow\langle n\rangle$ of pointed finite sets and for every $1 \leqslant j \leqslant n$ of a morphism $\otimes_{i \in t^{-1}(j)} M_{i} \otimes_{A} B \rightarrow N_{j}$ of $L^{\prime}$-modules.

It comes with a projection functor $\int \mathrm{dgRep}^{\otimes} \rightarrow \int \mathrm{dgLie}^{\mathrm{op}} \times \mathrm{Fin}^{*}$. We will say that a morphism in $\int d g R e p{ }^{\otimes}$ is a weak equivalence if the underlying maps of pointed finite sets, of cdga's and of dg-Lie algebras are identities and if the map dg-representations it contains is a quasi-isomorphism. Let us denote by $\int \mathbf{d g R e p}{ }^{\otimes}$ the localisation of $\int \operatorname{dgRep}^{\otimes}$ along weak equivalences. This defines a coCartesian fibration $p: \int \operatorname{dgRep}^{\otimes} \rightarrow \int \mathrm{dgLie}^{\mathrm{op}} \times$ Fin $^{*}$ (using once again [DAG-X, 2.4.19]).

Let now $\int \operatorname{dgMod}_{\mathrm{C}(-)}^{\otimes}$ be the following category

- An object is a family $\left(A, L, V_{1}, \ldots, V_{m}\right)$ with $A \in \operatorname{cdga}_{k}^{\leqslant 0}$, with $L \in \operatorname{dgLie}_{A}$ and with $V_{i} \in$ $\operatorname{dgMod}_{\mathrm{C}_{A} L}$.

- A morphism $\left(A, L, V_{1}, \ldots, V_{m}\right) \rightarrow\left(B, L^{\prime}, W_{1}, \ldots, W_{n}\right)$ is the datum of a map $(A, L) \rightarrow\left(B, L^{\prime}\right) \in$ $\int \mathrm{dgLie}^{\mathrm{op}}$, of a map of pointed finite sets $t:\langle m\rangle \rightarrow\langle n\rangle$ and for every $1 \leqslant j \leqslant n$ of a morphism of $\mathrm{C}_{B} L^{\prime}$-dg-modules $\otimes_{i \in t^{-1}(j)} V_{i} \otimes_{\mathrm{C}_{A} L} \mathrm{C}_{B} L^{\prime} \rightarrow W_{j}$.

We will say that a morphism in $\int d g R e p{ }^{\otimes}$ is a weak equivalence if the underlying maps of pointed finite sets, of cdga's and of dg-Lie algebras are identities and if the map of dg-modules it contains is a quasiisomorphism. Localising along weak equivalences, we get a coCartesian fibration of $(\infty, 1)$-categories $q: \int \operatorname{dgMod}_{\mathrm{C}(-)}^{\otimes} \rightarrow \int \mathrm{dgLie}^{\mathrm{op}} \times \mathrm{Fin}^{*}$.

Lemma 2.3.8. The above coCartesian fibrations $p$ and $q$ define functors

$$
\operatorname{dgRep}, \operatorname{dgMod}_{\mathrm{C}(-)}: \int \operatorname{dgLie}^{\mathrm{op}} \rightarrow \mathrm{Cat}_{\infty}^{\otimes, \mathbb{V}}
$$

Proof. For any object $(A, L) \in \int$ dgLie $^{\text {op }}$, the pulled back coCartesian fibration

$$
\int \operatorname{dgRep}^{\otimes} \underset{\int \operatorname{dgLie}^{\mathrm{op}} \times \mathrm{Fin}^{*}}{\times}\{(A, L)\} \times \mathrm{Fin}^{*} \rightarrow \mathrm{Fin}^{*}
$$

defines a symmetric monoidal structure on the $(\infty, 1)$-category $\operatorname{dgRep}_{A}(L)$ - see definition 0.0.2. The coCartesian fibration $p$ is therefore classified by a functor

$$
\int \mathrm{dgLie}^{\mathrm{op}} \rightarrow \mathbf{C a t}_{\infty}^{\otimes, \mathbb{V}}
$$

Moreover, this functor maps quasi-isomorphisms of dg-Lie algebras to equivalences. Hence it factors through a functor

$$
\text { dgRep : } \int \operatorname{dgLie}^{\mathrm{op}} \rightarrow \mathrm{Cat}_{\infty}^{\otimes, \mathbb{V}}
$$

The case of $\mathbf{d g M o d}_{\mathrm{C}(-)}$ is isomorphic.

We will now focus on building a natural transformation between those two functors. Let us build a functor $g: \int d g \operatorname{Rep}^{\otimes} \rightarrow \int \operatorname{dgMod}_{\mathrm{C}(-)}^{\otimes}$

- The image of an object $\left(A, L, M_{1}, \ldots, M_{m}\right)$ is the family $\left(A, L, V_{1}, \ldots, V_{m}\right)$ where $V_{i}$ is the $\mathrm{C}_{A} L$-dg-module

$$
g_{L}^{A}\left(M_{i}\right)=\underline{\operatorname{Hom}}_{L}\left(\mathcal{U}_{A}(A[\eta] \underset{A}{\otimes} L), M_{i}\right)
$$


- The image of an arrow $\bigotimes_{i \in t^{-1}(j)} M_{i} \otimes_{A} B \rightarrow N_{j}$ is the composition

$$
\begin{aligned}
\otimes g_{L}^{A}\left(M_{i}\right) \underset{\mathrm{C}_{A} L}{\otimes} \mathrm{C}_{B} L^{\prime} & \rightarrow g_{L}^{A}\left(\otimes M_{i}\right) \underset{\mathrm{C}_{A} L}{\otimes} \mathrm{C}_{B} L^{\prime} \\
& \rightarrow g_{L^{\prime}}^{B}\left(\otimes M_{i} \otimes{ }_{A} B\right) \\
& \rightarrow g_{L^{\prime}}^{B}(N)
\end{aligned}
$$

where the second map sends a tensor $\lambda \otimes \mu$ to $(\lambda \otimes$ id $) . \mu$ with

$$
\lambda \otimes \mathrm{id}: \mathcal{U}_{B}\left(B[\eta] \underset{B}{\otimes} L^{\prime}\right) \rightarrow \mathcal{U}_{B}(B[\eta] \underset{A}{\otimes} L)=\mathcal{U}_{A}(A[\eta] \underset{A}{\otimes} L) \underset{A}{\otimes} B \rightarrow\left(\otimes M_{i}\right) \underset{A}{\otimes} B
$$

The functor $g$ induces a functor of $(\infty, 1)$-categories

$$
g: \int \operatorname{dgRep}^{\otimes} \rightarrow \int \operatorname{dgMod}_{\mathrm{C}(-)}^{\otimes}
$$

which commutes with the coCartesian fibrations to $\int \mathrm{dgLie}^{\mathrm{op}} \times \mathrm{Fin}^{*}$.

Proposition 2.3.9. The functor $g$ admits a left adjoint $f$ relative to $\int \mathrm{dgLie}^{\mathrm{op}} \times \mathrm{Fin}^{*}$. There is therefore a commutative diagram of $(\infty, 1)$-categories

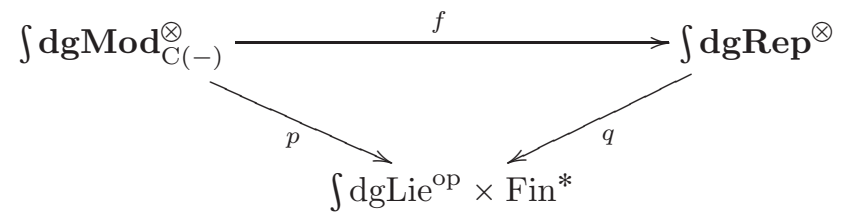

where $f$ preserves coCartesian morphisms. It follows that $f$ is classified by a (monoidal) natural transformation

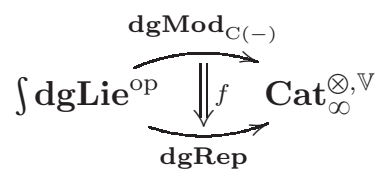

Proof. Whenever we fix $(A, L,\langle m\rangle)$ in $\int \mathrm{dgLie}^{\mathrm{op}} \times \mathrm{Fin}^{*}$, the functor $g$ restricted to the fibre categories admits a left adjoint (see lemma 2.3.3). Moreover when $(A, L) \rightarrow\left(B, L^{\prime}\right)$ is a morphism in $\int \mathrm{dgLie}^{\mathrm{op}}$, the following squares of monoidal functors commutes up to a canonical equivalence induced by the adjunctions

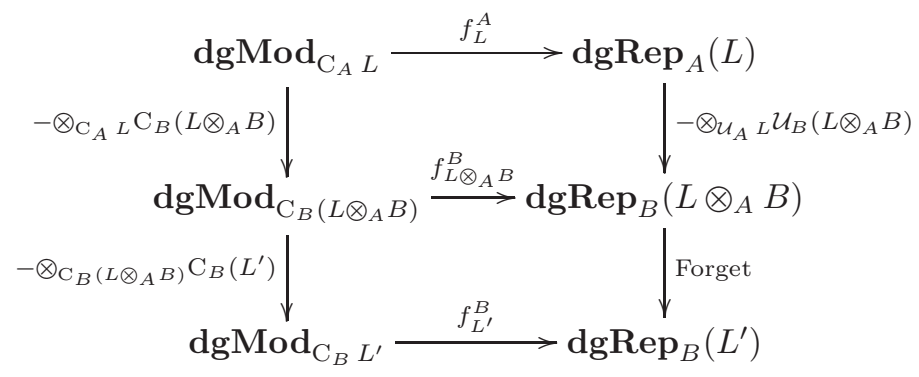

For any family $\left(V_{1}, \ldots, V_{m}\right)$ of $\mathrm{C}_{A} L$-dg-modules, the canonical morphism

$$
\left(\otimes f_{L}^{A}\left(V_{i}\right)\right) \underset{\mathrm{C}_{A} L}{\otimes} \mathrm{C}_{B} L^{\prime} \rightarrow f_{L^{\prime}}^{B}\left(\left(\otimes V_{i}\right) \underset{A}{\otimes} B\right)
$$

is hence an equivalence. This proves that $g$ satisfies the requirements of [HAlg, 8.3.2.11], admits a relative left adjoint $f$ which preserves coCartesian morphisms. 
Let us denote by $\int \operatorname{dgMod}^{\otimes}$ the category

- an object is a family $\left(A, M_{1}, \ldots, M_{m}\right)$ where $A \in \operatorname{cdga}_{k}^{\leqslant 0}$ and $M_{i} \in \operatorname{dgMod}_{A}$

- a morphism $\left(A, M_{1}, \ldots, M_{m}\right) \rightarrow\left(B, N_{1}, \ldots, N_{n}\right)$ is the datum of a morphism $A \rightarrow B$, of a map $t:\langle m\rangle \rightarrow\langle n\rangle$ of pointed finite sets and for any $1 \leqslant j \leqslant n$ of morphism of $A$-dg-modules

$$
\underset{i \in t^{-1}(j)}{\otimes} M_{i} \rightarrow N_{j}
$$

There is a natural projection $\int \operatorname{dgMod}^{\otimes} \rightarrow \operatorname{cdga}_{k}^{\leqslant 0} \times \mathrm{Fin}^{*}$. We have three functors

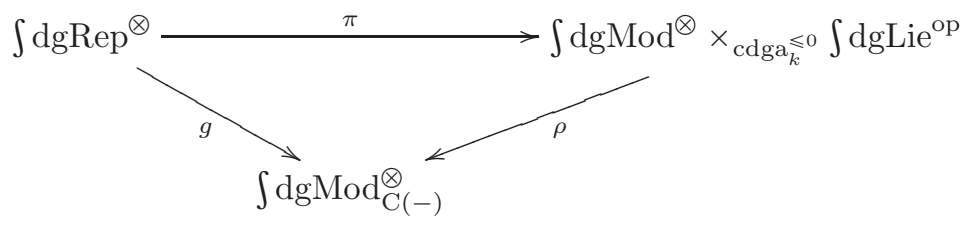

compatible with the projections to $\int d_{g L i e}{ }^{o p} \times F_{i n}^{*}$. The functor $\pi$ is defined by forgetting the Lie action, while $\rho$ maps an $A$-dg-module $M$ and an $A$-dg-Lie algebra $L$ to the $\mathrm{C}_{A} L$-dg-module $M$, where $\mathrm{C}_{A} L$ acts through the augmentation map $\mathrm{C}_{A} L \rightarrow A$. The above triangle does not commute, but we have a natural transformation $g \rightarrow \rho \pi$, defined on a triple $(A, L, V)$ by

$$
g(A, L, V)=\underline{\operatorname{Hom}}_{L}\left(\mathcal{U}_{A}\left(A[\eta] \otimes_{A} L\right), V\right) \stackrel{\mathrm{ev}_{1}}{\longrightarrow} V=\rho \pi(A, L, V)
$$

We check that this map indeed commutes with the $\mathrm{C}_{A} L$-action. We say that a map in $\int \mathrm{dgMod}^{\otimes}$ is a weak equivalence if the underlying maps of cdga's and of pointed sets are identities, and if the maps of dg-modules are quasi-isomorphisms. Localising the above diagram along weak equivalences, we get a tetrahedron

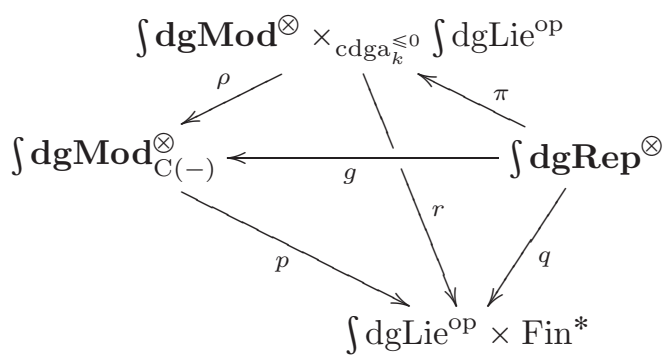

where $p, q$ and $r$ are coCartesian fibrations - see [DAG-X, 2.4.19] - where the upper face is filled with the natural transformation $g \rightarrow \rho \pi$ and where the other faces are commutative.

Lemma 2.3.10. The functor $\rho$ admits a relative left adjoint $\tau$ and the functor $\pi$ preserves coCartesian maps. Moreover, the natural transformation $\tau \rightarrow \pi f-$ induced by $g \rightarrow \rho \pi$ and by the adjunctions is an equivalence.

Remark 2.3.11. It follows from the above lemma the existence of natural transformations

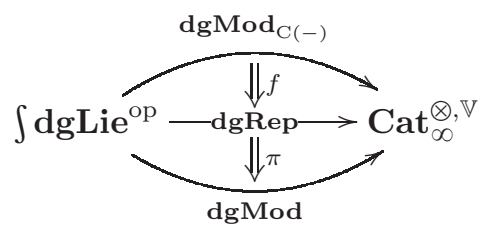

This lemma also describes the composite $\pi f$ as the base change functor along the augmentation maps $\mathrm{C}_{A} L \rightarrow A$. 
Proof. Let us first prove that $\rho$ admits a relative left adjoint $\tau$. For any pair $(A, L) \in \int \operatorname{dgLie}{ }^{\text {op }}$, the forgetful functor $\operatorname{dgMod}_{A} \rightarrow \operatorname{dgMod}_{\mathrm{C}_{A} L}$ admits a left adjoint, namely the base change functor along the augmentation map $\mathrm{C}_{A} L \rightarrow A$. This left adjoint is monoidal and commutes with base change. It therefore fulfil the assumptions of [HAlg, 8.3.2.11]. The induced natural transformation $\tau \rightarrow \pi f$ maps a triple $(A, L, V) \in \int \operatorname{dgMod}_{\mathrm{C}(-)}$ to the canonical map

$$
\tau_{L}^{A}(V)=V \underset{\mathrm{C}_{A} L}{\otimes} A \rightarrow V \underset{\mathrm{C}_{A} L}{\otimes} \mathcal{U}_{A}(L \underset{A}{\otimes} A[\eta])=\pi_{L}^{A} f_{L}^{A}(V)
$$

which is an equivalence of $A$-dg-modules.

Let us consider the functor of $(\infty, 1)$-categories

$$
\operatorname{dAff}_{k}^{\Delta^{2}} \rightarrow\left(\mathbf{C a t}_{\infty}^{\otimes, \mathbb{V}}\right)^{\mathrm{op}}
$$

mapping a sequence $X \rightarrow Y \rightarrow Z$ of derived affine schemes to the monoidal $(\infty, 1)$-category $\mathbf{Q} \operatorname{coh}(Y)$. We form the fibre product

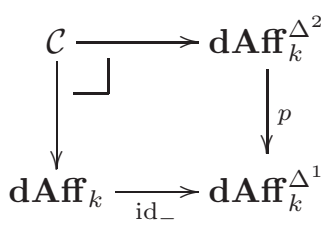

where $p$ is induced by the inclusion $(0 \rightarrow 2) \rightarrow(0 \rightarrow 1 \rightarrow 2)$. Finaly, we define $\mathcal{D}$ as the full subcategory of $\mathcal{C}$ spanned by those triangles $\operatorname{Spec} A \rightarrow \operatorname{Spec} B \rightarrow \operatorname{Spec} A$ where $B \in \mathbf{d g E x t}_{A}$. We get a functor

$$
F: \mathcal{D} \rightarrow\left(\mathbf{C a t}_{\infty}^{\otimes, \mathbb{V}}\right)^{\text {op }}
$$

mapping a trivial square-zero extension $B$ of $A$ to $\operatorname{dgMod}_{B}$. Note that the functor $\mathcal{D} \rightarrow \operatorname{dAff}_{k}$ is a Cartesian fibration classified by the functor $A \mapsto \mathbf{d g E x t}{ }_{A}^{\mathrm{op}}$.

Let us denote by $\oint \mathbf{d S t}^{\mathrm{f}} \rightarrow \mathbf{d} \mathbf{A f f}{ }_{k}$ the Cartesian fibration classified by the functor $\operatorname{Spec} A \mapsto$ $\mathbf{d S t}_{A}^{\mathrm{f}}=\mathcal{P}_{\Sigma}^{\mathrm{st}}\left(\mathbf{d g E x t} t_{A}^{\mathrm{op}}\right)$. The Yoneda natural transformation $\mathbf{d g E x t} \mathbf{t}^{\mathrm{op}} \rightarrow \mathbf{d S t}^{\mathrm{f}}$ defines a functor $\mathcal{D} \rightarrow \oint \mathbf{d S t}^{\mathrm{f}}$. We define

$$
\mathrm{L}_{\mathrm{qcoh}}: \oint \mathbf{d S t}^{\mathrm{f}} \rightarrow\left(\mathbf{C a t}_{\infty}^{\otimes, \mathbb{V}}\right)^{\mathrm{op}}
$$

as the left Kan extension of $F$ along $\mathcal{D} \rightarrow \oint \mathbf{d S t} \mathrm{t}^{\mathrm{f}}$.

Let now $X$ be a derived stack. The category $\mathbf{d S t} \mathbf{t}_{X}^{\mathrm{f}}$ defined in definition 2.1.4 is equivalent to the category of Cartesian sections $\phi$ as below - see [HTT, 3.3.3.2]

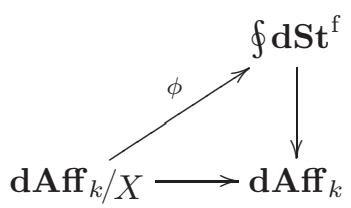

Definition 2.3.12. Let $X$ be a derived stack. We define the functor of derived category of formal stacks over $X$ :

$$
\mathrm{L}_{\mathrm{qcoh}}^{X}: \mathbf{d S t}_{X}^{\mathrm{f}} \simeq \mathrm{Fct}_{\mathbf{d A f f}_{k}}^{\mathrm{Cart}_{k}}\left(\mathbf{d} \mathbf{A f f} k / X, \oint \mathbf{d S t}^{\mathrm{f}}\right) \stackrel{\mathrm{L}_{\mathrm{qcoh}}}{\longrightarrow} \mathrm{Fct}\left(\mathbf{d A f f}_{k / X},\left(\mathbf{C a t}_{\infty}^{\otimes, \mathbb{V}}\right)^{\mathrm{op}}\right) \stackrel{\operatorname{colim}}{\longrightarrow}\left(\mathbf{C a t}_{\infty}^{\otimes, \mathbb{V}}\right)^{\mathrm{op}}
$$

If $Y \in \mathbf{d S t}_{X}^{\mathrm{f}}$ then $\mathrm{L}_{\mathrm{qcoh}}^{X}(Y)$ is called the derived category of the formal stack $Y$ over $X$. We can describe it more intuitively as the limit of symmetric monoidal $(\infty, 1)$-categories

$$
\mathrm{L}_{\mathrm{qcoh}}^{X}(Y) \simeq \lim _{\operatorname{Spec} A \rightarrow X} L_{\mathrm{qcoh}}^{\operatorname{Spec} A}\left(Y_{A}\right) \simeq \lim _{\operatorname{Spec} A \rightarrow X} \lim _{\substack{B \in \operatorname{dgExt}_{A} \\ \operatorname{Spec} B \rightarrow Y_{A}}} \operatorname{dgMod}_{B}
$$


where $Y_{A} \in \mathbf{d S t}_{A}^{\mathrm{f}}$ is the pullback of $Y$ along the morphism Spec $A \rightarrow X$. Remark that if $X=\operatorname{Spec} A$ and $Y=\operatorname{Spec} B$ with $B \in \mathbf{d g E x t}_{A}$, then $\mathrm{L}_{\mathrm{qcoh}}^{X}(Y)$ is nothing but $\operatorname{dgMod}_{B}$. section

The same way, the opposite category of dg-Lie algebras over $X$ is equivalent to that of coCartesian

We can thus define

$$
\operatorname{dgLie}_{X}^{\mathrm{op}} \simeq \mathrm{Fct}_{\text {cdga }_{k}^{<0}}^{\mathrm{coC}}\left(\left(\operatorname{dAff}_{k / X}\right)^{\mathrm{op}}, \int \operatorname{dgLie}^{\mathrm{op}}\right)
$$

Definition 2.3.13. Let $X$ be a derived stack. We define the functor of Lie representations over $X$ to be the composite functor $\operatorname{dgRep}_{X}: \mathbf{d g L i e}_{X}^{\mathrm{op}} \rightarrow \mathbf{C a t}_{\infty}^{\otimes, \mathbb{V}}$

$$
\operatorname{dgLi}_{X}^{\mathrm{op}} \longrightarrow \operatorname{Fct}\left(\left(\mathbf{d A f f}_{k} / X\right)^{\mathrm{op}}, \int \mathbf{d g L i e}^{\mathrm{op}}\right) \stackrel{\operatorname{dgRep}}{\longrightarrow} \operatorname{Fct}\left(\left(\mathbf{d A f f}_{k} / X\right)^{\mathrm{op}}, \mathbf{C a t}_{\infty}^{\otimes, \mathbb{V}}\right) \stackrel{\lim }{\longrightarrow} \mathbf{C a t}_{\infty}^{\otimes, \mathbb{V}}
$$

In particular for any $L \in \mathbf{d g L i e}{ }_{X}$, this defines a symmetric monoidal $(\infty, 1)$-category

$$
\operatorname{dgRep}_{X}(L)=\lim _{\operatorname{Spec} A \rightarrow X} \operatorname{dgRep}_{A}\left(L_{A}\right)
$$

where $L_{A} \in \mathbf{d g L i e}_{A}$ is the dg-Lie algebra over $A$ obtained by pulling back $L$.

Proposition 2.3.14. Let $X$ be a derived stack. There is a natural transformation

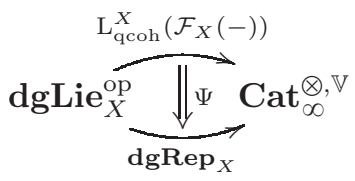

Moreover, for any $L \in \mathbf{d g L i e}{ }_{X}$, the induced monoidal functor $\mathrm{L}_{\mathrm{qcoh}}^{X}\left(\mathcal{F}_{X} L\right) \rightarrow \operatorname{dgRep}_{X}(L)$ is fully faithful and preserves colimits.

To prove this proposition, we will need the following

Lemma 2.3.15. The natural transformation $\mathcal{F}: \mathbf{d g L i e} \rightarrow \mathbf{d S t}^{\mathrm{f}}$ and the functor $\mathrm{L}_{\mathrm{qcoh}}: \oint \mathbf{d S t}^{\mathrm{f}} \rightarrow$ $\left(\mathbf{C a t}_{\infty}^{\otimes, \mathbb{V}}\right)^{\mathrm{op}}$ define a composite functor

$$
\phi: \int \operatorname{dgLie}^{\mathrm{op}} \stackrel{\int \mathcal{F}}{\longrightarrow}\left(\oint \mathbf{d S t}^{\mathrm{f}}\right)^{\mathrm{op}} \stackrel{\mathrm{L}_{\mathrm{qcoh}}}{\longrightarrow} \mathbf{C a t}_{\infty}^{\otimes, \mathbb{V}}
$$

There is a pointwise fully faithful and colimit preserving natural transformation $\phi \rightarrow \mathbf{d g R e p}$.

Proof. Let $\mathcal{E}$ denote the full subcategory of $\int \mathbf{d g L i e}^{\text {op }}$ such that the induced coCartesian fibration $\mathcal{E} \rightarrow \mathbf{c d g a}_{k}^{\leqslant 0}$ is classified by the subfunctor

$$
A \mapsto\left(\operatorname{dgLie}_{A}^{\mathrm{f}, \mathrm{ft}, \geqslant 1}\right)^{\mathrm{op}} \subset \operatorname{dgLie}_{A}^{\mathrm{op}}
$$

The functor $\phi$ is by construction the right Kan extension of its restriction $\psi$ to $\mathcal{E}$. Moreover, the restriction $\psi$ is by definition equivalent to the composite functor

$$
\mathcal{E} \longrightarrow \int \operatorname{dgLie}^{\mathrm{op}} \stackrel{\operatorname{dgMod}_{\mathrm{C}(-)}}{\longrightarrow} \mathrm{Cat}_{\infty}^{\otimes, \mathbb{V}}
$$

Using the natural transformation $\operatorname{dgMod}_{\mathrm{C}(-)} \rightarrow \operatorname{dgRep}$ from proposition 2.3.9, we get

$$
\alpha: \psi \rightarrow \operatorname{dgRep}_{\mid \mathcal{E}} \in \operatorname{Fct}\left(\mathcal{E}, \operatorname{Cat}_{\infty}^{\otimes, \mathbb{V}}\right)
$$

We will prove the following sufficient conditions. 
(i) The functor dgRep: $\int \mathbf{d g L i e}^{\mathrm{op}} \rightarrow \mathbf{C a t}_{\infty}^{\otimes, \mathbb{V}}$ is the right Kan extension of its restriction $\mathbf{d g R e p} \mathbf{p}_{\mid \mathcal{E}}$

(ii) The natural transformation $\alpha$ is pointwise fully faithful and preserves finite colimits.

Condition (ii) simply follows from proposition 2.3.6. To prove condition (i), it suffices to see that when $A$ is fixed, the functor

$$
\operatorname{dgRep}_{A}: \operatorname{dgLie}_{A}^{\mathrm{op}} \rightarrow \mathrm{Cat}_{\infty}^{\otimes, \mathbb{V}}
$$

commutes with sifted limits. This follows from [DAG-X, 2.4.32].

Proof (of proposition 2.3.14). Let $X$ be a derived stack and $L$ be a dg-Lie algebra over $X$. Recall that we can see $L$ as a functor

$$
L:\left(\mathbf{d A f f}_{k / X}\right)^{\mathrm{op}} \rightarrow \int \mathbf{d g L i e}^{\mathrm{op}}
$$

By definition, we have $\mathrm{L}_{\mathrm{qcoh}}^{X}\left(\mathcal{F}_{X} L\right)$ is the limit of the diagram

$$
\left(\mathbf{d A f f}_{k} / X\right)^{\mathrm{op}} \stackrel{L}{\longrightarrow} \int \mathbf{d g L i e}^{\mathrm{op}} \stackrel{\int \mathcal{F}}{\longrightarrow}\left(\oint \mathbf{d S t}^{\mathrm{f}}\right)^{\mathrm{op}} \stackrel{\mathrm{L}_{\mathrm{qcoh}}}{\longrightarrow} \mathbf{C a t}_{\infty}^{\mathbb{V}}
$$

while $\operatorname{dgRep}_{X}(L)$ is the limit of

$$
\left(\mathbf{d A f f}_{k} / X\right)^{\mathrm{op}} \stackrel{L}{\longrightarrow} \int \mathbf{d g L i e}^{\mathrm{op}} \stackrel{\text { dgRep }}{\longrightarrow} \mathbf{C a t}_{\infty}^{\mathbb{V}}
$$

We then deduce the result from lemma 2.3.15, since a limit of fully faithful (resp. colimit preserving) functor is so.

We can now prove the promised theorem 2.3.1.

Proof (of theorem 2.3.1). Let us first build a functor

$$
\nu_{X}: \operatorname{Qcoh}(X) \rightarrow \mathrm{L}_{\mathrm{qcoh}}^{X}\left((X \times X)^{\mathrm{f}}\right)
$$

Let us denote by $\mathcal{C}$ the $(\infty, 1)$-category of diagrams

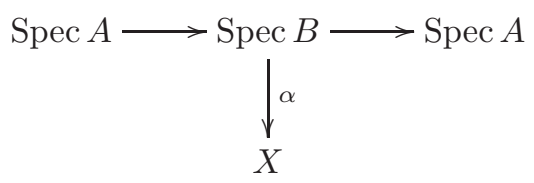

where $A \in \mathbf{c d g a}_{k}^{\leqslant 0}$ and $B \in \operatorname{dgExt}_{A}$. There is a natural functor $\mathcal{C}^{\text {op }} \rightarrow \mathbf{C a t}_{\infty}^{\otimes, \mathbb{V}}$ mapping a diagram as above to the monoidal $(\infty, 1)$-category $\operatorname{dgMod}_{B}$. Unwinding the definitions, we contemplate an equivalence of monoidal categories (recall (1) from definition 2.3.12)

$$
\mathrm{L}_{\mathrm{qcoh}}^{X}\left((X \times X)^{\mathrm{f}}\right) \simeq \lim _{\mathcal{C}} \operatorname{dgMod}_{B}
$$

The maps $\alpha$ as above induce (obviously compatible) pullback functors $\alpha^{*}: \mathbf{Q} \operatorname{coh}(X) \rightarrow \operatorname{dgMod}_{B}$. This construction defines the announced monoidal functor

$$
\nu_{X}: \operatorname{Qcoh}(X) \rightarrow \mathrm{L}_{\mathrm{qcoh}}^{X}\left((X \times X)^{\mathrm{f}}\right)
$$

Going back to definition 2.2.10 and definition 2.1.4, we have an adjunction co-unit

$$
\theta: \mathcal{F}_{X}\left(\ell_{X}\right)=\mathcal{F}_{X} \mathfrak{L}_{X}\left((X \times X)^{\mathrm{f}}\right) \rightarrow(X \times X)^{\mathrm{f}}
$$


Now using the functor from proposition 2.3.14, we get a composite functor

$$
\operatorname{Rep}_{X}: \operatorname{Qcoh}(X) \stackrel{\nu_{X}}{\longrightarrow} \mathrm{L}_{\mathrm{qcoh}}^{X}\left((X \times X)^{\mathrm{f}}\right) \stackrel{\theta^{*}}{\longrightarrow} \mathrm{L}_{\mathrm{qcoh}}^{X}\left(\mathcal{F}_{X}\left(\ell_{X}\right)\right) \stackrel{\Psi}{\longrightarrow} \operatorname{dgRep}_{X}\left(\ell_{X}\right)
$$

As every one of those functors is both monoidal and colimit preserving, so is Rep ${ }_{X}$. We still have to prove that $\operatorname{Rep}_{X}$ is a retract of the forgetful functor $\Theta_{X}: \operatorname{dgRep}_{X}\left(\ell_{X}\right) \rightarrow \mathbf{Q c o h}(X)$. We consider the composite functor $\Theta_{X} \operatorname{Rep}_{X}$

$$
\mathrm{Q} \operatorname{coh}(X) \stackrel{\nu_{X}}{\longrightarrow} \mathrm{L}_{\mathrm{qcoh}}^{X}\left((X \times X)^{\mathrm{f}}\right) \stackrel{\theta^{*}}{\longrightarrow} \mathrm{L}_{\mathrm{qcoh}}^{X}\left(\mathcal{F}_{X}\left(\ell_{X}\right)\right) \stackrel{\Psi}{\longrightarrow} \operatorname{dgRep}_{X}\left(\ell_{X}\right) \stackrel{\Theta_{X}}{\longrightarrow} \mathrm{Q} \operatorname{coh}(X)
$$

Unwinding the definitions, we see that $\Theta \Psi$ is the following limit

$$
\begin{aligned}
& \mathrm{L}_{\mathrm{qcoh}}^{X}\left(\mathcal{F}_{X}\left(\ell_{X}\right)\right)=\lim _{u: \operatorname{Spec} A \rightarrow X} \lim _{\substack{L \rightarrow u^{*} \ell_{X} \\
L \in \operatorname{dgLie}_{A}^{\text {fft, } f, \geqslant 1}}} \operatorname{dgMod}_{\mathrm{C}_{A} L} \\
& \operatorname{dgRep}_{X}\left(\ell_{X}\right)=\left.\lim _{u: \operatorname{Spec} A \rightarrow X}\right|_{\substack{L \rightarrow u^{*} \ell_{X} \\
L \in \operatorname{dgLie}_{A}^{f, f t, \geqslant 1}}} ^{f} \operatorname{dgRep}_{A}(L) \\
& \lim _{u: \operatorname{Spec} A \rightarrow X} \stackrel{\downarrow}{\pi}_{\operatorname{dgMod}}=\operatorname{Qcoh}(X)
\end{aligned}
$$

where $f$ and $\pi$ are induced by the natural transformation of remark 2.3.11. The composite functor $\pi f$ is equivalent to the pullback

$$
\mathrm{L}_{\mathrm{qcoh}}^{X}\left(\mathcal{F}_{X}\left(\ell_{X}\right)\right) \rightarrow \mathrm{L}_{\mathrm{qcoh}}^{X}(X) \simeq \mathrm{Q} \operatorname{coh}(X)
$$

along the unique morphism $X \rightarrow \mathcal{F}_{X}\left(\ell_{X}\right)$ of formal stacks over $X$ (seen as a formal stack, $X$ is initial in $\mathbf{d S t}_{X}^{\mathrm{f}}$. This map can be described as a colimit of augmentation maps $\mathrm{C}_{A} L \rightarrow A$ ). It follows that $\Theta_{X} \operatorname{Rep}_{X}$ is equivalent to the composite functor

$$
\mathrm{Q} \operatorname{coh}(X) \stackrel{\nu_{X}}{\longrightarrow} \mathrm{L}_{\mathrm{qcoh}}^{X}\left((X \times X)^{\mathrm{f}}\right) \stackrel{\alpha^{*}}{\longrightarrow} \mathrm{Q} \operatorname{coh}(X)
$$

where $\alpha$ is the morphism $X \rightarrow(X \times X)^{\mathrm{f}}$. Unwinding the definition of $\nu_{X}$, we see that this composite functor is equivalent to the identity functor of $\mathbf{Q} \operatorname{coh}(X)$.

\subsection{Atiyah class, modules and tangent maps}

Definition 2.4.1. Let Perf denote the derived stack of perfect complexes. It is defined as the stack mapping a cdga $A$ to the maximal $\infty$-groupoid in the $(\infty, 1)$-category $\operatorname{Perf}(A)$. For any derived stack $X$, we set $\underline{\operatorname{Perf}}(X)$ to be the maximal groupoid in $\operatorname{Perf}(X)$. It is equivalent to space of morphisms from $X$ to $\underline{\underline{\text { Perf }}}$ in $\mathbf{d S t}_{k}$.

Definition 2.4.2. Let $X$ be a derived Artin stack locally of finite presentation. Any perfect module

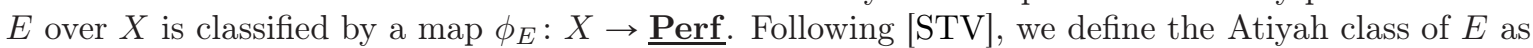
the tangent morphism of $\phi_{E}$

$$
\text { at }_{E}: \mathbb{T}_{X} \rightarrow \phi_{E}^{*} \mathbb{T}_{\text {Perf }}
$$


Remark 2.4.3. We will provide an equivalence $\phi_{E}^{*} \mathbb{T}_{\text {Perf }} \simeq \operatorname{End}(E)[1]$ in the proof of proposition 2.4.4. The Atiyah class of $E$ should be thought as the composition

$$
\text { at }_{E}: \mathbb{T}_{X}[-1] \rightarrow \phi_{E}^{*} \mathbb{T}_{\text {Perf }}[-1] \simeq \operatorname{End}(E)
$$

We will, at the end of this section, compare this definition of the Atiyah class with the usual one see proposition 2.4.7.

Proposition 2.4.4. Let $X$ be an algebraic stack locally of finite presentation. When $E$ is a perfect module over $X$, then the $\ell_{X}$-action on $E$ given by the theorem 2.3.1 is induced by the Atiyah class of E.

Lemma 2.4.5. Let $A \in \mathbf{c d g a}_{k}^{\leqslant 0}$ and $L \in \mathbf{d g L i e}_{A}^{\mathrm{f}, \mathrm{ft}, \geqslant 1}$. The functor

$$
f_{L}^{A}: \operatorname{dgMod}_{\mathrm{C}_{A}(L)} \rightarrow \operatorname{dgRep}_{A}(L)
$$

defined in lemma 2.3 .3 induces an equivalence

$$
\operatorname{Perf}\left(\mathrm{C}_{A} L\right) \stackrel{\sim}{\rightarrow} \operatorname{dgRep}_{A}(L) \times \operatorname{dgMod}_{A} \operatorname{Perf}(A)
$$

Proof. We proved in proposition 2.3.6 the functor $f_{L}^{A}$ to be fully faithful. Let us denote by $\mathcal{C}$ the image category

$$
\mathcal{C}=f_{L}^{A}\left(\operatorname{Perf}\left(\mathrm{C}_{A}(L)\right)\right)
$$

Since $f_{L}^{A}$ is exact, the category $\mathcal{C}$ is stable by shifts in $\operatorname{dgRep}_{A}(L)$. Let us first prove that $\mathcal{C}$ contains any representation whose underlying dg-module is projective of finite type (ie a retract of some $A^{n}$ ). Let $P$ be a projective of finite type dg-module over $A$. An action of $L$ on $P$ amounts a morphism $\kappa: M \rightarrow \operatorname{End}(P)$, where $M \in \operatorname{dgMod}_{A}^{\mathrm{ffft}} \geqslant 1$ such that $L=$ Free $_{A} M$. Such a map corresponds to a choice of finitely many elements in $\operatorname{End}(P)$ of positive cohomological degree. The cdga $A$ is by assumption cohomologically concentrated in non-positive degree. So is $\operatorname{End}(P)$, as a projective dgmodule over $A$. The map $\kappa$ is hence (non canonically) homotopic to 0 . Every $L$-action on $P$ is trivial. Moreover, the trivial action on $P$ is given by $f_{L}^{A}\left(\mathrm{C}_{A} L \otimes_{A} P\right)$.

Let now $d \in \mathbb{N}$. Let us assume that any $F \in \operatorname{dgRep}_{A}(L)$ whose underlying dg-module is perfect and of tor-amplitude contained in $[-d, 0]$ belongs to $\mathcal{C}$. Recall that the case $d=0$ is the projective case (see [TV, 2.22]). Let $E \in \operatorname{dgRep}_{A}(L)$ be a representation. We assume its underlying dg-module $\underline{E}$ is perfect of tor-amplitude contained in $[-d-1,0]$. Using loc. cit., there exists an exact sequence $N \rightarrow \underline{E} \rightarrow F$ where $N$ is a projective $A$-dg-module of finite type and $Q$ is a perfect complex of tor-amplitude contained in $[-d-1,-1]$. We will build a lift $N \rightarrow E$ in $\operatorname{dgRep}_{A}(L)$ of the map $N \rightarrow \underline{E}$. The $L$-action on $E$ is given by a morphism $M \rightarrow \operatorname{End}(\underline{E})$ where $M \in \operatorname{dgMod}_{A}^{\mathrm{f}, \mathrm{ft}, \geqslant 1}$ such that $L=$ Free $_{A}(M)$. A lift $N \rightarrow E$ of $N \rightarrow \underline{E}$ is equivalent to an homotopy $\alpha$ making the following diagram commutative

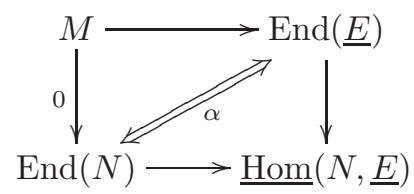

The module $N$ is a retract of $A^{n}$ for some $n$. It follows that $\underline{\operatorname{Hom}}(N, \underline{E})$ is a retract of $\underline{E}^{n} \simeq$ $\underline{\operatorname{Hom}}\left(A^{n}, \underline{E}\right)$. The dg-module $\underline{\operatorname{Hom}}(N, \underline{E})$ is thus cohomologically concentrated in non-positive degree. Since $M$ is generated by objects in positive degree, the mapping $\operatorname{space}^{\operatorname{Map}_{A}}(M, \underline{\operatorname{Hom}}(N, \underline{E}))$ is connected and an homotopy $\alpha$ as above exists (but is not uniquely determined). We obtain from what precedes a map $\beta: N \rightarrow E$ in $\operatorname{dgRep}_{A}(L)$ lifting $N \rightarrow \underline{E}$. Let $F$ denote the cofibre of $\beta$ in $\operatorname{dgRep}_{A}(L)$. Since the forgetful functor $\operatorname{dgRep}_{A}(L) \rightarrow \operatorname{dgMod}_{A}$ is exact, the underline $\operatorname{dg}$-module of $F$ is equivalent to $Q$ and hence of tor-amplitude contained in $[-d-1,-1]$. By assumption, the representation $Q[-1]$ belongs to $\mathcal{C}$. Since $\mathcal{C}$ is stable by shifts, we have $Q \in \mathcal{C}$. The category $\mathcal{C}$ is 
also stable by extensions and we have $E \in \mathcal{C}$. By induction, we get that every representation of $L$ whose underlying module is perfect belongs to $\mathcal{C}$. Reciprocally, any representation of $\mathcal{C}$ has a perfect underlying complex.

Definition 2.4.6. Let $X$ be a derived stack and let $Y$ be a formal stack over $X$. We define the full subcategory $\mathrm{L}_{\mathrm{pe}}^{X}(Y)$ of $\mathrm{L}_{\mathrm{qcoh}}^{X}(Y)$

$$
\mathrm{L}_{\mathrm{pe}}^{X}(Y)=\lim _{\operatorname{Spec} A \rightarrow X} \lim _{\substack{B \in \operatorname{dgExt}_{A} \\ \operatorname{Spec} B \rightarrow Y_{A}}} \operatorname{Perf}_{B} \longrightarrow \lim _{\operatorname{Spec} A \rightarrow X} \lim _{\substack{B \in \operatorname{dgExt}_{A} \\ \operatorname{Spec} B \rightarrow Y_{A}}} \operatorname{dgMod}_{B} \simeq \mathrm{L}_{\mathrm{qcoh}}^{X}(Y)
$$

Proof (of proposition 2.4.4). The sheaf $E$ corresponds to a morphism $\phi_{E}: X \rightarrow \underline{\text { Perf. Its Atiyah }}$ class is the tangent morphism

$$
\text { at }_{E}: \mathbb{T}_{X}[-1] \rightarrow \phi_{E}^{*} \mathbb{T}_{\text {Perf }}[-1]
$$

In our setting, we get a Lie tangent map (theorem 2.0.1)

$$
\text { at }_{E}: \ell_{X} \rightarrow \phi_{E}^{*} \underline{\underline{\text { Perf }}}
$$

Using remark 2.2.11, we get an equivalence $\left.\phi_{E}^{*} \underline{\underline{\text { Perf }}} \simeq \mathfrak{L}_{X}(X \times \underline{\text { Perf }})^{\mathrm{f}}\right)$. The dg-Lie algebra $\phi_{E}^{*} \underline{\underline{\text { Perf }}}$ hence represents the presheaf on $\mathbf{d g L i} \mathbf{i}_{X}$

$$
\begin{aligned}
& \operatorname{Map}\left(-, \mathfrak{L}_{X}\left((X \times \underline{\text { Perf }})^{\mathrm{f}}\right)\right) \simeq \operatorname{Map}\left(\mathcal{F}_{X}(-),(X \times \underline{\text { Perf }})^{\mathrm{f}}\right) \\
& \simeq \lim _{u: \operatorname{Spec} A \rightarrow X} \lim _{\substack{L \in \mathbf{d g L i} \mathbf{e}_{A}^{\mathrm{f}, \mathrm{ft}}, \geqslant 1 \\
L \rightarrow u^{*}(-)}} \operatorname{Map}\left(\operatorname{Spec}\left(\mathrm{C}_{A} L\right),(\operatorname{Spec} A \times \underline{\mathbf{P e r f}})^{\mathrm{f}}\right) \\
& \simeq \lim _{u: \operatorname{Spec} A \rightarrow X} \lim _{\substack{L \in \operatorname{dgLie} \\
L \rightarrow u_{A}^{\text {fft }}, \geqslant 1 \\
L(-)}} \underline{\operatorname{Perf}}\left(\mathrm{C}_{A} L\right) \underset{\underline{\operatorname{Perf}}(A)}{\times}\left\{u^{*} E\right\} \\
& \simeq \operatorname{Gpd}\left(\mathrm{L}_{\mathrm{pe}}^{X}\left(\mathcal{F}_{X}(-)\right) \underset{\operatorname{Perf}(X)}{ } \times\{E\}\right)
\end{aligned}
$$

where Gpd associates to any $(\infty, 1)$-category its maximal groupoid. Note that the equivalence between the second and third lines follows from remark 2.2.6. On the other hand $\mathfrak{g l}(E)$ - the dg-Lie algebra of endomorphisms of $E$ - represents the functor

$$
\operatorname{Gpd}\left(\operatorname{dgRep}_{X}(-) \times\{E\}\right)
$$

We get from proposition 2.3.14 a morphism $\phi_{E}^{*} \ell_{\text {Perf }} \rightarrow \mathfrak{g l}(E)$ of dg-Lie algebras over $X$. Restricting to an affine derived scheme $s: \operatorname{Spec} A \rightarrow X$, we get that $s^{*} \phi_{E}^{*} \ell_{\text {Perf }}$ and $s^{*} \mathfrak{g l}(E) \simeq \mathfrak{g l}\left(s^{*} E\right)$ respectively represent the functors $\left(\mathbf{d g L i e}_{A}^{\mathrm{f}, \mathrm{ft}, \geqslant 1}\right)^{\text {op }} \rightarrow$ sSets

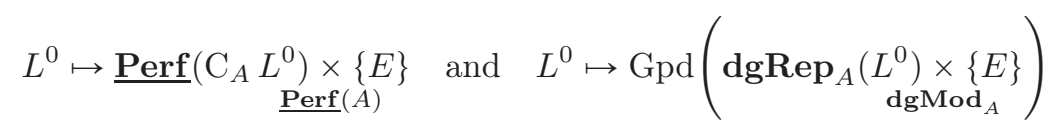

The natural transformation induced between those functors is the one of lemma 2.4.5 and is thus an equivalence. We therefore have

$$
\text { at }_{E}: \ell_{X} \rightarrow \mathfrak{g l}(E)
$$

and hence an action of $\ell_{X}$ on $E$. This construction corresponds to the one of theorem 2.3.1 through the equivalence $\underline{\operatorname{Perf}}(X) \simeq \operatorname{Map}(X, \underline{\text { Perf }})$. 
We will now focus on comparing our definition 2.4.2 of the Atiyah class with a more usual one. Let $X$ be a smooth variety. Let us denote by $X^{(2)}$ the infinitesimal neighbourhood of $X$ in $X \times X$ through the diagonal embedding. We will also denote by $i$ the diagonal embedding $X \rightarrow X^{(2)}$ and by $p$ and $q$ the two projections $X^{(2)} \rightarrow X$. We have an exact sequence

$$
i_{*} \mathbb{L}_{X} \rightarrow \mathcal{O}_{X^{(2)}} \rightarrow i_{*} \mathcal{O}_{X}
$$

classified by a morphism $\alpha: i_{*} \mathcal{O}_{X} \rightarrow i_{*} \mathbb{L}_{X}[1]$. The Atiyah class of a quasi-coherent sheaf $E$ is usually obtained from this extension class by considering the induced map - see for instance [KM]

$$
E \simeq p_{*}\left(i_{*} \mathcal{O}_{X} \otimes q^{*} E\right) \rightarrow p_{*}\left(i_{*} \mathbb{L}_{X}[1] \otimes q^{*} E\right) \simeq \mathbb{L}_{X}[1] \otimes E
$$

From the map $\alpha$, we get a morphism $i^{*} i_{*} \mathcal{O}_{X} \rightarrow \mathbb{L}_{X}[1]$. Dualising we get

$$
\beta: \mathbb{T}_{X}[-1] \rightarrow \underline{\operatorname{Hom}}_{\mathcal{O}_{X}}\left(i^{*} i_{*} \mathcal{O}_{X}, \mathcal{O}_{X}\right) \simeq p_{*} i_{*} \underline{\operatorname{Hom}}_{\mathcal{O}_{X}}\left(i^{*} i_{*} \mathcal{O}_{X}, \mathcal{O}_{X}\right) \simeq p_{*} \underline{\operatorname{Hom}}_{\mathcal{O}_{X(2)}}\left(i_{*} \mathcal{O}_{X}, i_{*} \mathcal{O}_{X}\right)
$$

The right hand side naturally acts on the functor $i^{*} \simeq p_{*}\left(-\otimes_{\mathcal{O}_{X(2)}} i_{*} \mathcal{O}_{X}\right)$ and hence on $i^{*} q^{*} \simeq$ id. This action, together with the map $\beta$, associates to any perfect module $E$ a morphism $\mathbb{T}_{X}[-1] \otimes E \rightarrow E$. It corresponds to a map $E \rightarrow E \otimes \mathbb{L}_{X}[1]$ which is equivalent to the Atiyah class in the sense of (3).

Proposition 2.4.7. Let $X$ be a smooth algebraic variety and let $E$ be a perfect complex on $X$. The Atiyah class of $E$ in the sense of definition 2.4.2 and the construction (3) are equivalent to one another.

Proof. We first observe that $\left(X^{(2)}\right)^{\mathrm{f}}$ is locally a trivial square zero extension: there exists a covering $a$ : Spec $A \rightarrow X$ such that $u^{*}\left(X^{(2)}\right)^{\mathrm{f}} \simeq \operatorname{Spec}\left(A \oplus \mathbb{L}_{X, a}\right)$ with $A$ a noetherian ring. As consequences

- The derived category $\mathrm{L}_{\mathrm{qcoh}}^{X}\left(\left(X^{(2)}\right)^{\mathrm{f}}\right)$ is equivalent to $\mathrm{Qcoh}\left(X^{(2)}\right)$.

- The tangent Lie algebra $\mathfrak{L}_{X}\left(\left(X^{(2)}\right)^{\mathrm{f}}\right)$ is locally equivalent to the free Lie algebra generated by $\mathbb{T}_{X}[-1]$ (see corollary 1.4.11 and lemma 1.4.12).

We moreover have a commutative diagram:

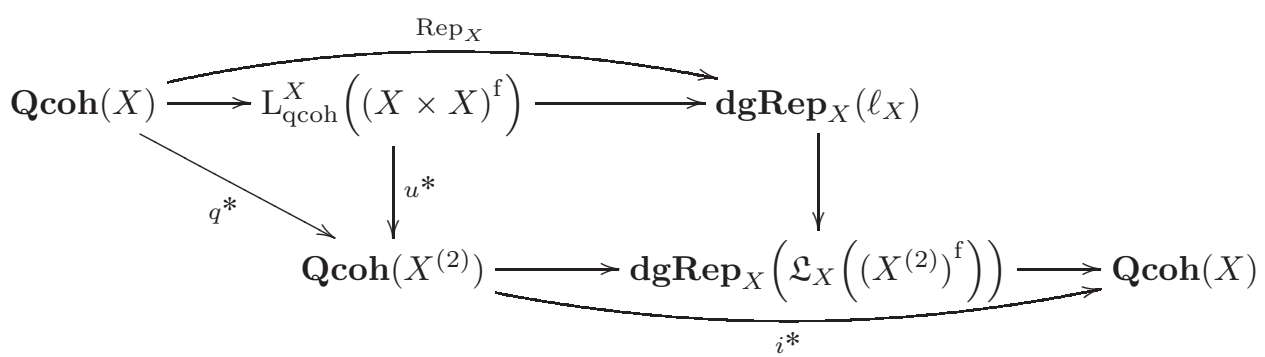

where $i: X \rightarrow X^{(2)}$ is the inclusion and $u$ is the natural morphism $\left(X^{(2)}\right)^{\mathrm{f}} \rightarrow(X \times X)^{\mathrm{f}}$.

From what precedes, the Atiyah class arises from an action on $i^{*}$, and we can thus focus on the composite

$$
\operatorname{Qcoh}\left(X^{(2)}\right) \longrightarrow \operatorname{dgRep}_{X}\left(\mathfrak{L}_{X}\left(\left(X^{(2)}\right)^{\mathrm{f}}\right)\right) \longrightarrow \mathbf{Q} \operatorname{coh}(X)
$$

which can be studied locally. Let thus $a: \operatorname{Spec} A \rightarrow X$ be as above. Let us denote by $L$ the $A$-dg-Lie algebra Free ${ }_{A}\left(\mathbb{T}_{X, a}[-1]\right) \simeq a^{*} \mathfrak{L}_{X}\left(\left(X^{(2)}\right)^{\mathrm{f}}\right)$. Pulling back on $A$ the functors above, we get

$$
\operatorname{dgMod}_{\mathrm{C}_{A} L} \stackrel{f_{L}^{A}}{\longrightarrow} \operatorname{dgRep}_{A}(L) \longrightarrow \operatorname{dgMod}_{A}
$$


where $f_{L}^{A}$ is given by the action of $L$ on $\mathcal{U}_{A}\left(L \otimes_{A} A[\eta]\right)$ through the natural inclusion. On the other hand, the universal Atiyah class $\alpha$ defined above can be computed as follows

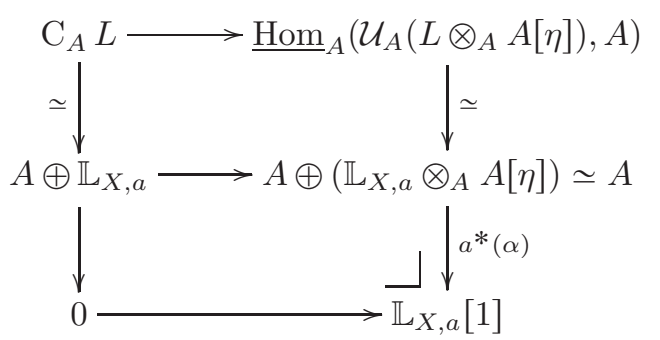

The universal Atiyah class is thus dual to the inclusion $\mathbb{T}_{X, a}[-1] \rightarrow \mathcal{U}_{A}\left(L \otimes_{A} A[\eta]\right)$. It follows that the action defined by the functor $f_{L}^{A}$ is indeed given by the Atiyah class. We now conclude using proposition 2.4.4.

\subsection{Adjoint represention}

In this subsection, we will focus on the following statement.

Proposition 2.5.1. Let $X$ be a derived Artin stack. The $\ell_{X}$-module $\operatorname{Rep}_{X}\left(\mathbb{T}_{X}[-1]\right)$ is equivalent to the adjoint representation of $\ell_{X}$.

The above proposition, coupled with proposition 2.4.4, implies that the bracket of $\ell_{X}$ is as expected given by the Atiyah class of the tangent complex. To prove it, we will need a few constructions.

Lemma 2.5.2. Let $A \in \operatorname{cdga}_{k}^{\leqslant 0}$ and $L \in \operatorname{dgLie}_{A}$. To any $A$-dg-Lie algebra $L^{\prime}$ with a morphism $\alpha: L \rightarrow L^{\prime}$ we associate the underlying representation $\psi_{L}^{A}\left(L^{\prime}\right)$ of $L$-ie the $A$-dg-module $L^{\prime}$ with the action of $L$ through the morphism $\alpha$. The functor $\psi_{L}^{A}$ preserves quasi-isomorphisms. It induces a functor between the localised $(\infty, 1)$-categories, which admits a left adjoint $\phi_{L}^{A}$ :

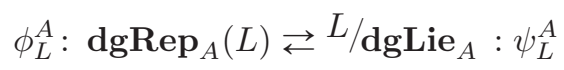

Proof. The functor $\psi_{L}^{A}$ preserves small limits and both its ends are presentable $(\infty, 1)$-categories. Since both $\operatorname{dgRep}_{A}(L)$ and $\mathbf{d g L i e}_{A}$ are monadic over $\operatorname{dgMod}_{A}$, the functor $\psi_{L}^{A}$ is accessible for the cardinal $\omega$. The result follows from [HTT, 5.5.2.9]

Lemma 2.5.3. Let $A$ be a cdga and $L_{0}$ be a dg-Lie algebra over $A$. There is a natural transformation

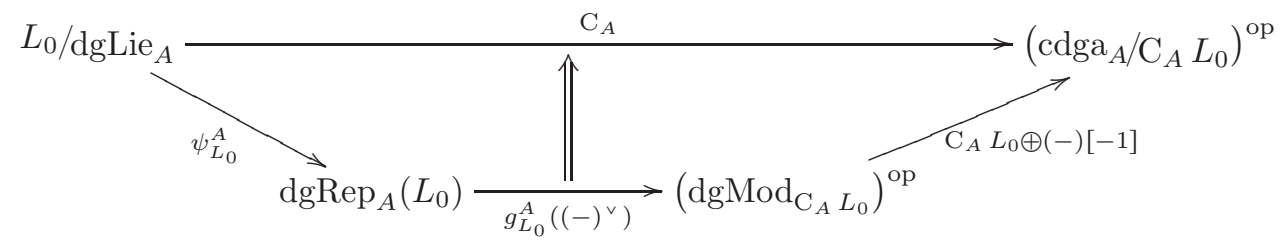

where $g_{L_{0}}^{A}$ was defined in lemma 2.3.3 and where $\mathrm{C}_{A} L_{0} \oplus(-)[-1]$ is a trivial square zero extension functor.

Proof. Let $\alpha: L_{0} \rightarrow L$ be a morphism of $A$-dg-Lie algebras. The composite morphism

$$
\operatorname{Sym}_{A}\left(L_{0}[1]\right) \underset{A}{\otimes} L[1] \stackrel{\alpha}{\rightarrow} \operatorname{Sym}_{A}(L[1]) \underset{A}{\otimes} L[1] \rightarrow \operatorname{Sym}_{A}(L[1])
$$

induces a morphism

$$
\underline{\operatorname{Hom}}_{A}\left(\operatorname{Sym}_{A}(L[1]), A\right) \rightarrow \underline{\operatorname{Hom}}_{A}\left(\operatorname{Sym}\left(L_{0}[1]\right) \otimes L[1], A\right) \simeq \underline{\operatorname{Hom}}_{A}\left(\operatorname{Sym}_{A}\left(L_{0}[1]\right), L^{\vee}[-1]\right)
$$


Using remark 2.3.4, this defines a map of graded modules $\delta_{L}: \mathrm{C}_{A} L \rightarrow g_{L_{0}}^{A}\left(L^{\vee}\right)[-1]$. Let us prove that it commutes with the differentials. Recall the notations $S_{i}$ and $T_{i j}$ from remark 1.4.2. We compute on one hand

$$
\begin{aligned}
\delta(d \xi)\left(\eta \cdot x_{1} \otimes \ldots \otimes \eta \cdot x_{n}\right) & \left(\eta \cdot y_{n+1}\right)=\sum_{i \leqslant n+1}(-1)^{S_{i}+1} \xi\left(\eta \cdot y_{1} \otimes \ldots \otimes \eta \cdot d y_{i} \otimes \ldots \otimes \eta \cdot y_{n+1}\right) \\
& +\sum_{i<j \leqslant n+1}(-1)^{T_{i j}} \xi\left(\eta \cdot\left[y_{i}, y_{j}\right] \otimes \eta \cdot y_{1} \otimes \ldots \otimes \widehat{\eta \cdot y_{i}} \otimes \ldots \otimes \widehat{\eta \cdot y_{j}} \otimes \ldots \otimes \eta \cdot y_{n+1}\right) \\
& +d\left(\xi\left(\eta \cdot y_{1} \otimes \ldots \otimes \eta \cdot y_{n+1}\right)\right)
\end{aligned}
$$

where $y_{i}$ denotes $\alpha x_{i}$, for any $i \leqslant n$. On the other hand, we have

$$
\begin{aligned}
(d(\delta \xi))\left(\eta \cdot x_{1} \otimes \ldots \otimes \eta \cdot x_{n}\right) & =\sum_{i \leqslant n}(-1)^{S_{i}+1} \delta \xi\left(\eta \cdot x_{1} \otimes \ldots \otimes \eta \cdot d x_{i} \otimes \ldots \otimes \eta \cdot x_{n}\right) \\
& +\sum_{i<j \leqslant n}(-1)^{T_{i j}} \delta \xi\left(\eta \cdot\left[x_{i}, x_{j}\right] \otimes \eta \cdot x_{1} \otimes \ldots \otimes \widehat{\eta \cdot x_{i}} \otimes \ldots \otimes \widehat{\eta \cdot x_{j}} \otimes \ldots \otimes x_{n}\right) \\
& +\sum_{i \leqslant n}(-1)^{\left(\left|x_{i}\right|+1\right) S_{i}} x_{i} \bullet \delta \xi\left(\eta \cdot x_{1} \otimes \ldots \otimes \widehat{\eta \cdot x_{i}} \otimes \ldots \otimes \eta \cdot x_{n}\right) \\
& +d\left(\delta \xi\left(\eta \cdot x_{1} \otimes \ldots \otimes \eta \cdot x_{n}\right)\right)
\end{aligned}
$$

where $\bullet$ denotes the action of $L_{0}$ on $L^{\vee}$. We thus have

$$
\begin{aligned}
(d(\delta \xi))( & \left.\eta \cdot x_{1} \otimes \ldots \otimes \eta \cdot x_{n}\right)\left(\eta \cdot y_{n+1}\right)=\sum_{i \leqslant n}(-1)^{S_{i}+1} \xi\left(\eta \cdot y_{1} \otimes \ldots \otimes \eta \cdot d y_{i} \otimes \ldots \otimes \eta \cdot y_{n} \otimes \eta \cdot y_{n+1}\right) \\
& +\sum_{i<j \leqslant n}(-1)^{T_{i j}} \xi\left(\eta \cdot\left[y_{i}, y_{j}\right] \otimes \eta \cdot y_{1} \otimes \ldots \otimes \widehat{\eta \cdot y_{i}} \otimes \ldots \otimes \widehat{\eta \cdot y_{j}} \otimes \ldots \otimes \eta \cdot y_{n} \otimes \eta \cdot y_{n+1}\right) \\
& +\sum_{i \leqslant n}(-1)^{\left(\left|y_{i}\right|+1\right) S_{i}+\left(\left|y_{i}\right|-1+S_{n+1}\right)\left|y_{i}\right|} \xi\left(\eta \cdot y_{1} \otimes \ldots \otimes \widehat{\eta \cdot y_{i}} \otimes \ldots \eta \cdot y_{n} \otimes \eta \cdot\left[y_{n+1}, y_{i}\right]\right) \\
& +d\left(\delta \xi\left(\eta \cdot x_{1} \otimes \ldots \otimes \eta \cdot x_{n}\right)\right)\left(\eta \cdot y_{n+1}\right)
\end{aligned}
$$

Now computing the difference $\delta(d \xi)\left(\eta \cdot x_{1} \otimes \ldots \otimes \eta \cdot x_{n}\right)\left(\eta \cdot y_{n+1}\right)-(d(\delta \xi))\left(\eta \cdot x_{1} \otimes \ldots \otimes \eta \cdot x_{n}\right)\left(\eta \cdot y_{n+1}\right)$ we get

$$
\begin{aligned}
(-1)^{S_{n+1}+1} \xi\left(\eta \cdot y_{1} \otimes \ldots \otimes \eta \cdot y_{n} \otimes \eta \cdot d y_{n+1}\right) \\
+d\left(\xi\left(\eta \cdot y_{1} \otimes \ldots \otimes \eta \cdot y_{n+1}\right)\right)-d\left(\delta \xi\left(\eta \cdot x_{1} \otimes \ldots \otimes \eta \cdot x_{n}\right)\right)\left(\eta \cdot y_{n+1}\right)=0
\end{aligned}
$$

It follows that $\delta_{L}$ is indeed a morphism of complexes $\mathrm{C}_{A} L \rightarrow g_{L_{0}}^{A}\left(L^{\vee}\right)[-1]$. It is moreover $A$-linear. One checks with great enthusiasm that it is a derivation. This construction is moreover functorial in $L$ and we get the announced natural transformation.

Let us define the category $\int * / \operatorname{dgLie}$ as follows

- An object is a triple $\left(A, L, L \rightarrow L_{1}\right)$ where $A \in \operatorname{cdga}_{k}^{\leqslant 0}$ and $L \rightarrow L_{1} \in \operatorname{dgLie}_{A}$.

- A morphism $\left(A, L, L \rightarrow L_{1}\right) \rightarrow\left(B, L^{\prime}, L^{\prime} \rightarrow L_{1}^{\prime}\right)$ is the data of

- A morphism $A \rightarrow B$ in $\operatorname{cdga}_{k}^{\leqslant 0}$,

- A commutative diagram

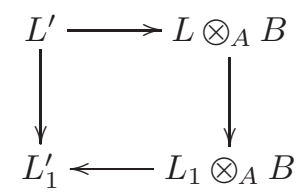


This category comes with a coCartesian projection to $\int * / \mathrm{dgLie} \rightarrow \int d g \operatorname{Lie}^{\text {op }}$. The forgetful functor $L / \operatorname{dgLie}_{A} \rightarrow \operatorname{dgRep}_{A}(L)$ define a functor Ad such that the following triangle commutes

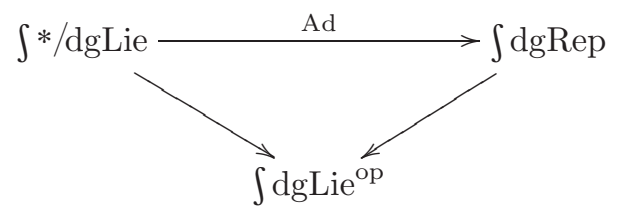

Let us define the category $\int(\operatorname{cdga} / \mathrm{C}(-))^{\text {op }}$ as follows

- An object is a triple $(A, L, B)$ where $(A, L) \in \int \mathrm{dgLie}^{\text {op }}$ and $B$ is a cdga over $A$ with a map $B \rightarrow \mathrm{C}_{A} L$

- A morphism $(A, L, B) \rightarrow\left(A^{\prime}, L^{\prime}, B^{\prime}\right)$ is a commutative diagram

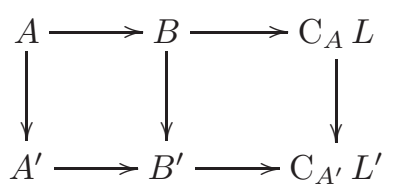

where $\mathrm{C}_{A} L \rightarrow \mathrm{C}_{A^{\prime}} L^{\prime}$ is induced by a given morphism $L^{\prime} \rightarrow L \otimes{ }_{A}^{\mathbb{L}} A^{\prime}$.

Let us remark here that $\mathrm{C}$ induces a functor $\chi: \int * / \operatorname{dgLie} \rightarrow \int(\operatorname{cdga} / \mathrm{C}(-))^{\text {op }}$ which commutes with the projections to $\int \operatorname{dgLie}{ }^{\mathrm{op}}$. The construction $\operatorname{dgRep}_{A}(L) \rightarrow\left(\operatorname{cdga}_{A} / \mathrm{C}_{A} L\right)^{\mathrm{op}}$

$$
V \mapsto \mathrm{C}_{A} L \oplus g_{L}^{A}\left(V^{\vee}\right)[1]
$$

defines a functor

$$
\theta: \int \operatorname{dgRep} \rightarrow \int(\operatorname{cdga} / \mathrm{C}(-))^{\mathrm{op}}
$$

and lemma 2.5.3 gives a natural transformation $\theta \mathrm{Ad} \rightarrow \chi$. Localising along quasi-isomorphisms, we get a thetaedron

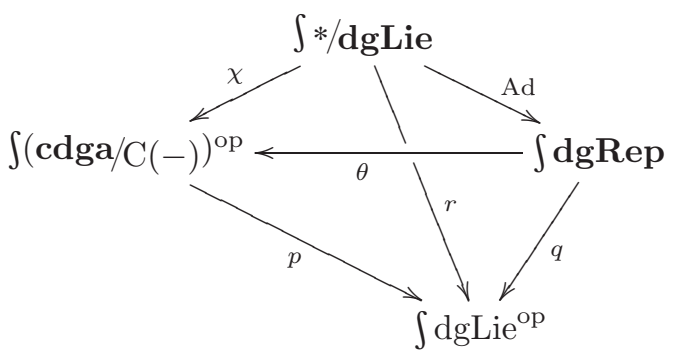

where the upper face is filled with the natural transformation $\theta \mathrm{Ad} \rightarrow \chi$ and the other faces are commutative.

Lemma 2.5.4. The functor Ad admits a relative left adjoint $\phi$ over $\int \mathrm{dgLie}{ }^{\mathrm{op}}$. Moreover, the induced natural transformation $\theta \rightarrow \theta \operatorname{Ad} \phi \rightarrow \chi \phi$ is an equivalence.

Proof. The first statement is a consequence of lemma 2.5.2 and [HAlg, 8.3.2.11]. To prove the second one, we fix a pair $(A, L) \in \int d g L i e^{o p}$ and study the induced natural transformation

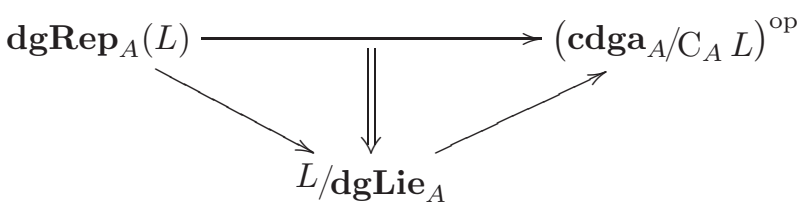


The category $\operatorname{dgRep}_{A}(L)$ is generated under colimits of the free representations $\mathcal{U}_{A} L \otimes N$, where $N \in \operatorname{dgMod}_{A}$. Both the upper and the lower functors map colimits to limits. Since $\phi_{L}^{A}\left(\mathcal{U}_{A} L \otimes N\right) \simeq$ $L \amalg \operatorname{Free}_{A}(N)$, we can restrict to proving that the induced morphism

$$
\mathrm{C}_{A} L \oplus N^{\vee}[-1] \rightarrow \mathrm{C}_{A} L \oplus g_{L}^{A}\left(\left(\mathcal{U}_{A} L \otimes N\right)^{\vee}[-1]\right)
$$

is an equivalence. We have the following morphism between exact sequences

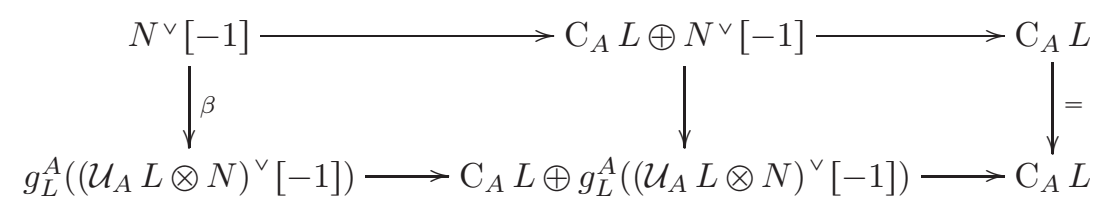

Since the functors $(-)^{\vee}$ and $g_{L}^{A}\left(\left(\mathcal{U}_{A} L \otimes-\right)^{\vee}[-1]\right)$ from $\mathbf{d g} \operatorname{Mod}{ }_{A}$ to $\operatorname{dgMod}_{A}^{\text {op }}$ are both left adjoint to the same functor, the morphism $\beta$ is an equivalence.

Let us now consider the functor

$$
\int \operatorname{dgMod}_{\mathrm{C}(-)}^{\mathrm{f}, \mathrm{ft}, \geqslant 1} \longrightarrow \int \operatorname{dgMod}_{\mathrm{C}(-)} \stackrel{f}{\longrightarrow} \int \operatorname{dgRep}
$$

Remark 2.5.5. Duality and proposition 2.3.6 make the composite functor

$$
\int \operatorname{dgMod} \operatorname{deft}_{\mathrm{C}(-)}^{\mathrm{f}, \geqslant 1} \stackrel{f}{\longrightarrow} \int \operatorname{dgRep} \stackrel{\theta}{\longrightarrow} \int(\operatorname{cdga} / \mathrm{C}(-))^{\mathrm{op}}
$$

equivalent to the functor $(A, L, M) \mapsto \mathrm{C}_{A} L \oplus M^{\vee}[-1]$.

Remark 2.5.6. The composite functor

$$
\begin{aligned}
& \int \operatorname{dgMod}_{\mathrm{C}(-)}^{\mathrm{f}, \mathrm{ft}, \geqslant 1} \underset{\int \mathrm{dgLie}{ }^{o p}}{\times} \int\left(\operatorname{dgLie}^{\mathrm{f}, \mathrm{ft}, \geqslant 1}\right)^{\mathrm{op}} \rightarrow \int \operatorname{dgRep}_{\int \operatorname{dgLie}}^{\times} \underset{\times}{\times} \int\left(\operatorname{dgLie}^{\mathrm{f}, \mathrm{ft}, \geqslant 1}\right)^{\mathrm{op}} \\
& \rightarrow \int * / \mathbf{d g L i e} \underset{\int d g L i e^{o p}}{\times} \int\left(\operatorname{dgLie}^{\mathrm{f}, \mathrm{ft}, \geqslant 1}\right)^{\text {op }}
\end{aligned}
$$

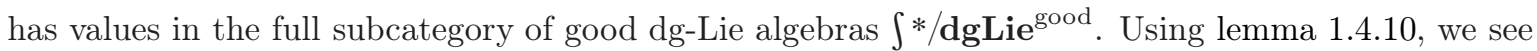
that the functor

$$
\int \operatorname{dgMod}_{\mathrm{C}(-)}^{\mathrm{f}, \mathrm{ft}, \geqslant 1} \underset{\int \mathrm{dgLie} e^{o p}}{\times} \int\left(\mathrm{dgLie}^{\mathrm{f}, \mathrm{ft}, \geqslant 1}\right)^{\mathrm{op}} \rightarrow \int(\operatorname{cdga} / \mathrm{C}(-))^{\mathrm{op}} \underset{\int \mathrm{dgLie}}{\times} \underset{\mathrm{op}}{\times} \int\left(\operatorname{dgLie}{ }^{\mathrm{f}, \mathrm{ft}, \geqslant 1}\right)^{\mathrm{op}}
$$

preserves coCartesian morphisms. We finally get a natural transformation

$$
\int\left(\operatorname{dgLie}^{\mathrm{f}, \mathrm{ft}, \geqslant 1} \frac{\operatorname{dgMod}_{\mathrm{C}(-)}^{\mathrm{f}, \mathrm{ft}, \geqslant 1}}{\frac{\mathrm{op}}{(\operatorname{cdga} / \mathrm{C}(-))^{\mathrm{op}}}} \operatorname{Cat}_{\infty}^{\mathbb{V}}\right.
$$

There is also a Yoneda natural transformation $(\mathbf{c d g a} / \mathrm{C}(-))^{\mathrm{op}} \rightarrow \operatorname{Spec}(\mathrm{C}(-)) / \mathbf{d S t}^{\mathrm{f}}$ and we get

$$
\xi: \operatorname{dgMod} \operatorname{Cot}_{\mathrm{C}(-)}^{\mathrm{ft}, \geqslant 1} \rightarrow \operatorname{Spec}(\mathrm{C}(-)) / \mathbf{d S t}^{\mathrm{f}}
$$

Let us recall remark 2.1.2. It defines a natural transformation

$$
\zeta: \operatorname{dgMod}_{\mathrm{C}(-)}^{\mathrm{f}, \mathrm{ft}, \geqslant 1} \times \Delta^{1} \rightarrow \operatorname{Spec}(\mathrm{C}(-)) / \mathbf{d S t}^{\mathrm{f}}
$$

such that $\zeta(-, 0) \simeq \mathcal{F} \phi f$ and $\zeta(-, 1) \simeq \xi \simeq h \theta f$. 
We are at last ready to prove proposition 2.5.1.

Proof (of proposition 2.5.1). Extending the preceding construction by sifted colimits, we get a natural transformation $\beta: \mathrm{L}_{\mathrm{qcoh}}(\mathcal{F}(-)) \times \Delta^{1} \rightarrow \mathcal{F}(-) / \mathbf{d S t}^{\mathrm{f}}$ of functors $\int \mathbf{d g L i e}^{\mathrm{op}} \rightarrow \mathbf{C a t}_{\infty}^{\mathbb{V}}$. Let now $X$ denote an Artin derived stack locally of finite presentation. We get a functor

$$
\beta_{X}: \mathrm{L}_{\mathrm{qcoh}}^{X}\left(\mathcal{F}_{X} \ell_{X}\right) \times \Delta^{1} \rightarrow \mathcal{F}_{X}\left(\ell_{X}\right) / \mathbf{d S t}_{X}^{\mathrm{f}}
$$

On the one hand, the functor $\beta_{X}(-, 0)$ admits a right adjoint, namely the functor

$$
\mathcal{F}_{X}\left(\ell_{X}\right) / \mathbf{d S t}_{X}^{\mathrm{f}} \stackrel{\mathfrak{L}_{X}}{\longrightarrow} \ell_{X} / \mathbf{d g L i e} \operatorname{Lid}_{X} \stackrel{\operatorname{Ad}_{X}}{\longrightarrow} \operatorname{dgRep}_{X}\left(\ell_{X}\right) \stackrel{g_{X}}{\longrightarrow} \mathrm{L}_{\mathrm{qcoh}}^{X}\left(\mathcal{F} \ell_{X}\right)
$$

On the other hand, using remark 2.5.5 and remark 2.2.6, we have an equivalence of functors

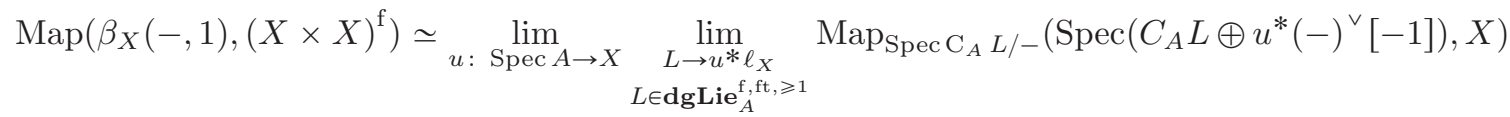

$$
\begin{aligned}
& \simeq \lim _{u: \operatorname{Spec} A \rightarrow X} \lim _{\substack{L \rightarrow u^{*} \ell_{X} \\
L \in \mathbf{d g L i e}_{A}^{\text {fft, }}, \geq 1}} \operatorname{Map}_{\mathbf{d g M o d}_{\mathrm{C}_{A} L}}\left(-, \nu_{L}^{*} \mathbb{T}_{X}[-1]\right)
\end{aligned}
$$

where $\nu_{L}$ is the map $\operatorname{Spec}\left(\mathrm{C}_{A} L\right) \simeq \mathcal{F}_{A}(L) \rightarrow X$ induced by $L \rightarrow u^{*} \ell_{X} \simeq \mathfrak{L}_{A}\left((\operatorname{Spec} A \times X)^{\mathrm{f}}\right)$. We get $\operatorname{Map}\left(\beta_{X}(-, 1),(X \times X)^{\mathrm{f}}\right) \simeq \operatorname{Map}\left(-, \nu_{X} \mathbb{T}_{X}[-1]\right)$ where $\nu_{X}$ is the functor

$$
\nu_{X}: \operatorname{Qcoh}(X) \rightarrow \mathrm{L}_{\mathrm{qcoh}}^{X}\left((X \times X)^{\mathrm{f}}\right)
$$

defined in the proof of theorem 2.3.1. The natural transformation $\beta_{X}: \beta_{X}(-, 0) \rightarrow \beta_{X}(-, 1)$ therefore induces a morphism

$$
\nu_{X}\left(\mathbb{T}_{X}[-1]\right) \rightarrow g_{X} \operatorname{Ad}_{X} \mathfrak{L}_{X}\left((X \times X)^{\mathrm{f}}\right) \simeq g_{X} \operatorname{Ad}_{X}\left(\ell_{X}\right)
$$

and hence, by adjunction, a morphism $\operatorname{Rep}_{X}\left(\mathbb{T}_{X}[-1]\right)=f_{X} \nu_{X}\left(\mathbb{T}_{X}[-1]\right) \rightarrow \operatorname{Ad}_{X}\left(\ell_{X}\right)$. It now suffices to test on the underlying quasi-coherent sheaves on $X$, that it is an equivalence. Both the left and right hand sides are equivalent to $\mathbb{T}_{X}[-1]$.

\section{References}

[Coh] Paul M. Cohn: A remark on the Birkhoff-Witt theorem. Journal of the London Math. Soc., (38) pp.193-203, 1963.

[DAG-X] Jacob LuRIE: Derived algebraic geometry X: Formal Moduli Problems. 2011, available at [http://www.math.harvard.edu/ lurie/papers/DAG-X.pdf].

[HAG2] Bertrand TOËN and Gabriele Vezzosi: Homotopical algebraic geometry II: geometric stacks and applications. Memoirs of the AMS, 193(902) pp.257-372, 2008.

[HAlg] Jacob LURIE: Higher algebra. Feb. 15, 2012, available at [http://www.math.harvard.edu/ Iurie/papers/HigherAlgebra.pdf].

[Hin] Vladimir Hinich: Dg-coalgebras as formal stacks. Journal of pure and applied algebra, 162 pp.209-250, 2001.

[HTT] Jacob LuRIE: Higher topos theory, volume 170 of Annals of Mathematics Studies. Princeton University Press, 2009. 
[Kap] Mikhail Kapranov: Rozansky-Witten invariants via Atiyah class. Compositio Math., 115(1) pp.71-113, 1999.

[KM] Alexander Kuznetsov and Dimitri Markushevich: Symplectic structures on moduli spaces of sheaves via the Atiyah class. Journal of Geometry and Physics, 59 pp.843-860, 2009 .

[Pri] Jonathan Pridham: Unifying derived deformation theories. Advances in Mathematics, 224(3) pp.772-826, 2010.

[SS] Stefan Schwede and Brooke E. Shipley: Algebras and modules in monoidal model categories. Proc. London Math. Soc., 80 pp.491-511, 2000.

[STV] Timo SchÜrg, Bertrand ToËN and Gabriele Vezzosi: Derived algebraic geometry, determinants of perfect complexes, and applications to obstruction theories for maps and complexes. 2013.

[TV] Bertrand TOËN and Michel VAquiÉ: Moduli of objects in dg-categories. Annales scientifiques de l'ENS, 40 pp.387-444, 2007. 